\title{
Solução numérica do modelo de Maxwell para escoamentos tridimensionais com superfícies livres
}

Renato Aparecido Pimentel da Silva

Orientador: Prof. Dr. Murilo Francisco Tomé

Dissertação apresentada ao Instituto de Ciências Matemáticas e de Computação - ICMC-USP, como parte dos requisitos para obtenção do título de Mestre em Ciências - Ciências de Computação e Matemática Computacional.

\section{"VERSÃO REVISADA APÓS A DEFESA"}

Data da Defesa:

$20 / 04 / 2007$

Visto do Orientador:

USP - São Carlos

Maio/2007 

Solução numérica do modelo de Maxwell para escoamentos tridimensionais com superfícies livres

Renato Aparecido Pimentel da Silva 

Aos meus pais,

Sonia e Cicero 

Meus sinceros agradecimentos a todas as pessoas que colaboraram direta e indiretamente com a realização deste trabalho.

Agradeço a meus pais em especial, porque a eles devo tudo o que sou e o que consegui. Sempre estiveram presentes, me apoiando em todas as etapas de minha vida.

Ao meu orientador, o Prof. Dr. Murilo Francisco Tomé, pela amizade, ajuda e colaboração, essenciais para o bom desenvolvimento do projeto.

A todos os docentes do Instituto de Ciências Matemáticas e de Computação da USP-São Carlos, que ministraram as disciplinas do meu curso de graduação, bem como as do programa de Mestrado, por trasmitirem e fortalecerem o conhecimento necessário para que este trabalho fosse realizado.

A todo o pessoal do LCAD - Laboratório de Computação de Alto Desempenho, em especial ao Alex Gimenes, ao Cláudio e ao João Paulo, pelos momentos de descontração, apoio e amizade. Também aproveito este espaço para lembrar meus amigos da graduação, em especial à Cibele, à Márcia, ao Ottone e à Alessandra.

Aos Profs. Drs. Fernando Tavares de Pinho e Manuel António Moreira Alves, da Faculdade de Engenharia da Universidade do Porto, em Portugal, pelo acolhimento e pela colaboração. Também agradeço à "malta" do CEFT, o Adélio, o Alexandre e o Pedro, por sua amizade.

Um agradecimento especial à Dra. Gilcilene Sanchez de Paulo - a minha grande amiga Gil - por sua amizade, companhia e força, essenciais durante meu período no Velho Mundo.

Agradeço à FAPESP e à CAPES pelo fomento à pesquisa e apoio financeiro prestados durante o Curso.

Por fim, agradeço principalmente a Deus, por todas as oportunidades que surgiram em minha vida. 



\section{Resumo}

Este trabalho apresenta um método numérico para simular escoamentos viscoelásticos tridimensionais com superfícies livres governados pela equação constitutiva de Maxwell. O método numérico é uma extensão da técnica newtoniana GENSMAC3D para escoamentos viscoelásticos. As equações governantes para escoamentos incompressíveis cartesianos isotérmicos são apresentadas em detalhes. O tratamento do tensor não-newtoniano em contornos rígidos tridimensionais é apresentado em detalhes, bem como o cálculo da condição de contorno na superfície livre. As equações governantes são resolvidas pelo método de diferenças finitas numa malha deslocada tridimensional. O fluido é modelado pela técnica das partículas marcadoras e tratado como uma superfície linear por partes. O método numérico desenvolvido foi implementado no sistema Freeflow3D, e resultados numéricos obtidos na simulação de escoamentos tridimensionais governados pela equação constitutiva de Maxwell são apresentados. Adicionalmente, apresentamos uma validação mostrando a convergência do método desenvolvido nesse trabalho. 



\begin{abstract}
This work presents a numerical method for solving three-dimensional viscoelastic flows with free surfaces governed by the Maxwell constitutive equation. The numerical method is an extension of the Newtonian technique GENSMAC3D to viscoelastic flows. The governing equations for Cartesian incompressible isothermic flows are presented in details. The treatment of the non-Newtonian tensor on three-dimensional rigid boundaries is given in details as well as the calculation of the boundary conditions on the free surface. The governing equations are solved by a finite difference method using a three-dimensional staggered grid. The fluid is described by marker particles and is represented by a piecewise linear surface. The numerical method developed in this work was implemented into the Freeflow3D system, and numerical results obtained from the simulation of complex three-dimensional flows are presented. Additionally, we present validation results and demonstrate the convergence of the method by performing mesh refinement.
\end{abstract}





\section{Lista de Figuras}

1.1 Contornos rígidos paralelos ao plano $x y$ (a), $x z$ (b) e $y z(\mathrm{c}) . \quad$. 19

3.1 Célula computacional utilizada na discretização por diferenças finitas. . . . . . . . . . . . . . . . . 32

3.2 Tipos de célula no domínio computacional. . . . . . . . . . . 34

3.3 Estêncil utilizado para obter $\phi_{A}$ e $\phi_{B}$ através de CUBISTA. . 41

3.4 Tipos de superfícies lineares: 1D (a), 2D (b) e 3D (c) . . . . . 46

3.5 Célula S com superfície linear do tipo 1D. . . . . . . . . 47

3.6 Célula S com superfície linear do tipo 2D. . . . . . . . . . 49

3.7 Célula S com superfície linear do tipo 3D. . . . . . . . . . 51

3.8 Configurações de células B com apenas a face $(k+1 / 2)$ (a) ou apenas a face $(k-1 / 2)$ (b) em contato com célula F ou S. . . 53

3.9 Configurações de células $\mathrm{B}$ com apenas a face $(j+1 / 2)$ (a) ou apenas a face $(j-1 / 2)$ (b) em contato com uma face da célula F ou S. . . . . . . . . . . . . . . . . . . 5 55

3.10 Configurações de células B com apenas a face $(i+1 / 2)$ (a) ou apenas a face $(i-1 / 2)$ (b) em contato com célula $\mathrm{F}$ ou $\mathrm{S}$. . 57

3.11 Configurações de células B com duas (a) ou três faces (b) em contato com células F ou S. . . . . . . . . . . . . . 59

3.12 Configurações de células I com a face $(i+1 / 2)$ (a) ou $(i-1 / 2)$ em contato com célula F ou S. . . . . . . . . . . . . 62

3.13 Configurações de células $\mathrm{O}$ com a face $(i+1 / 2)$ (a) ou $(i-1 / 2)$ (b) em contato com célula F ou S. . . . . . . . . . . 62

4.1 Solução numérica do escoamento de um fluido de Maxwell num tubo: descrição do domínio computacional. . . . . . . . . . 67 
4.2 Evolução do enchimento do tubo. . . . . . . . . . . . . . . 70

4.3 Visualização de $w$ na saída de fluido e ao longo do tubo em

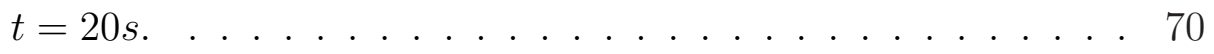

4.4 Visualização de $S^{z z}$ na saída de fluido e ao longo do tubo em $t=20 s \ldots \ldots \ldots \ldots \ldots \ldots \ldots$

4.5 Decaimento do erro relativo com o refinamento da malha. . . . 71

4.6 Comparação entre a solução analítica e as soluções numéricas na saída do fluido, para $x=0 \ldots \ldots$. . . . . . . . 72

5.1 Domínio computacional utilizado na simulação numérica do jato oscilante. . . . . . . . . . . . . . . . . 75

5.2 Domínio computacional utilizado na simulação numérica do inchamento do extrudado. . . . . . . . . . . . 77

5.3 Resultados quantitativos das simulações do inchamento do extrudado. . . . . . . . . . . . . . . . . . . 79

5.4 Taxa de inchamento $S_{r}$ : comparação entre os resultados obtidos e resultados teóricos de Tanner. . . . . . . . . . . . . 80

5.5 Simulação para $R e=0.5$ (Problema 1). À esquerda, fluido newtoniano; à direita, fluido de Maxwell. . . . . . . . . . . . 81

5.6 (cont.) Simulação para $R e=0.5$ (Problema 1). À esquerda, fluido newtoniano; à direita, fluido de Maxwell. . . . . . . . . 82

5.7 Simulação para $R e=1.3$ (Problema 2). À esquerda, fluido newtoniano; à direita, fluido de Maxwell. . . . . . . . . . . 83

5.8 (cont.) Simulação para $R e=1.3$ (Problema 2). À esquerda, jato newtoniano; à direita, jato viscoelástico. . . . . . . . . . 84

5.9 Visualização das simulações do inchamento do extrudado com aumento do número de Weissenberg, para instantes $t=5 \mathrm{~s}$, $7.5 \mathrm{~s}, 10 \mathrm{~s}$ e $15 \mathrm{~s}$, respectivamente. . . . . . . . . . 85

5.10 (cont.) Visualização das simulações do inchamento do extrudado com aumento do número de Weissenberg, para instantes $t=5 \mathrm{~s}, 7.5 \mathrm{~s}, 10 \mathrm{~s}$ e $15 \mathrm{~s}$, respectivamente. . . . . . . . 86 


\section{Lista de Tabelas}

4.1 Erro relativo na seção transversal adjacente à saída de fluido. . 72

5.1 Dados de entrada para as simulações numéricas do problema do jato oscilante. . . . . . . . . . . . . . . . 74

5.2 Resultados obtidos nas simulações do problema do inchamento do extrudado. . . . . . . . . . . . . . . . . 78 


\section{Sumário}

$\begin{array}{ll}\text { Introdução } & 1\end{array}$

Trabalhos relacionados e estado da arte . . . . . . . . . . . 2

Organização da dissertação . . . . . . . . . . . . . . . . . . . 5

1 Equações governantes $\quad 7$

1.1 Forma cartesiana tridimensional . . . . . . . . . . . . . . . . 9

1.2 Adimensionalização . . . . . . . . . . . . . . . . . . . . . . . . 11

1.3 Condições de contorno . . . . . . . . . . . . . . . . . 15

1.4 Cálculo do tensor $\mathbf{S}$ em contornos rígidos . . . . . . . . . . 16

1.4.1 Contornos rígidos paralelos ao plano $x y \ldots \ldots . . . .18$

1.4.2 Contornos rígidos paralelos ao plano $x z \ldots \ldots . . . .23$

1.4.3 Contornos rígidos paralelos ao plano yz . . . . . . 24

1.5 Condições de contorno em superfícies livres . . . . . . . . . . . . . . . . . 26

2 Método numérico $\quad 28$

2.1 Algoritmo computacional . . . . . . . . . . . . . . . 28

3 Aproximação por diferenças finitas 32

3.1 Classificação das células . . . . . . . . . . . . . . . . . . 33

3.2 Aproximação da equação de conservação de quantidade de movimento . . . . . . . . . . . . . . . . . . 33

3.3 Aproximação da equação de Poisson . . . . . . . . . . . . . . 36

3.4 Aproximação da equação da velocidade final . . . . . . . . . . . 37

3.5 Aproximação da equação da pressão . . . . . . . . . . . . . . . 37 
3.6 Aproximação da equação constitutiva . . . . . . . . . . . . . 38

3.7 Discretização dos termos convectivos . . . . . . . . . . . . . 39

3.8 Aproximação das condições de contorno na superfície livre . . 45

3.9 Aproximação do tensor não-newtoniano $\mathbf{S}$ em contornos rígidos 52

3.9.1 Contornos paralelos ao plano $x y$. . . . . . . . . 53

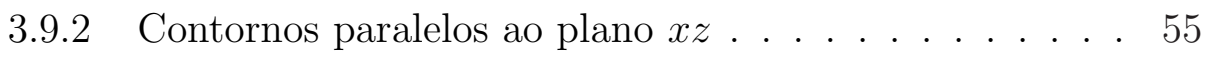

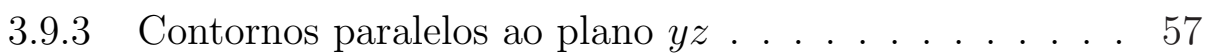

3.9.4 Células de canto . . . . . . . . . . . . . . . . 59

3.9.5 Aproximação das derivadas das velocidades . . . . . . . 60

3.10 Aproximação do tensor $\mathbf{S}$ em entradas e saídas de fluido . . . 61

3.11 Aproximação do tensor taxa de deformação D . . . . . . . . . 63

3.11.1 Aproximação de $\mathbf{D}$ nas células F e S . . . . . . . . . . 64

3.11.2 Aproximação de $\mathbf{D}$ nas células de contorno . . . . . . . 65

3.11.3 Aproximação de $\mathbf{D}$ em entradas e saídas de fluido . . . 66

$\begin{array}{lll}4 & \text { Resultados de validação } & 67\end{array}$

5 Simulação numérica de escoamentos viscoelásticos com superfícies livres $\quad 73$

5.1 Simulação numérica do jato oscilante . . . . . . . . . . . 73

5.2 Simulação numérica do inchamento do extrudado . . . . . . . 76

6 Considerações finais e conclusões $\quad 87$

$\begin{array}{lr}\text { Referências bibliográficas } & 89\end{array}$ 



\section{Introdução}

Ao longo dos últimos séculos, um dos grandes desafios dos estudiosos e cientistas é a investigação do movimento de fluidos, ou seja, dos líquidos e dos gases. Isto se deve ao fato de que, uma vez que os fluidos não têm uma forma definida, eles interagem entre si e com os corpos sólidos em contato durante seu movimento. Esta interação é denominada escoamento dos fluidos. O ramo da mecânica dos fluidos responsável pela tarefa de estudar o escoamento dos fluidos é a dinâmica dos fluidos, complementada pela estática dos fluidos, que estuda suas propriedades e seu comportamento quando em repouso. A partir da década de 1950, o computador digital mostrou-se uma ferramenta eficaz para esta área, e dessa forma, surgiu a dinâmica dos fluidos computacional, conhecida por seu acrônimo da língua inglesa CFD, que permitiu grandes avanços na pesquisa do movimento de fluidos. A dinâmica dos fluidos computacional consiste na utilização de métodos numéricos e algoritmos computacionais para resolver e analisar problemas que envolvem escoamentos de fluidos.

Na literatura estão disponíveis diversos resultados que tratam de escoamentos confinados, em que há um único fluido escoando e interagindo com um sólido que o contém, de forma que o fluido preenche completamente o interior de uma cavidade. Problemas que envolvem dois ou mais fluidos em contato entre si apresentam como desafio a representação da superfície livre, isto é, a interface entre dois ou mais fluidos, que movimenta-se continuamente com o tempo. Entretanto, o grande número de aplicações comerciais e industriais relacionados a escoamentos com superfícies livres tem motivado ao longo das últimas décadas diversos pesquisadores em CFD a concentrar esforços em tais problemas. Podemos citar como exemplos de tais aplicações a extrusão, mol- 
dagem e injeção de plásticos e ligas metálicas, o enchimento de contêineres e o processamento de embalagens na indústria petrolífera e alimentícia, dentre outros. Neste trabalho, vamos tratar de escoamentos viscosos de um fluido viscoelástico em contato com o ar, que é um fluido de densidade e viscosidade desprezíveis. Dessa forma, a superfície livre representará a interface entre o fluido e a atmosfera.

A simulação de escoamentos com superfície livre impõe alguns desafios que devem ser tratados criteriosamente:

- Representação da superfície livre: como representar computacionalmente a superfície, isto é, quais as estruturas de dados computacionais que podem ser utilizadas para modelar a superfície livre ao longo do domínio computacional;

- Quais as condições de contorno para as variáveis do processo (por exemplo, tensões, velocidade e pressão) a serem impostas na superfície livre, que sejam coerentes do ponto de vista físico;

- Como será a evolução da superfície livre ao longo do tempo, isto é, de que maneira reposicionar a superfície a cada passo de tempo da simulação.

\section{Trabalhos relacionados e estado da arte}

O método MAC (Marker-and-Cell), desenvolvido por Harlow e Welch na década de 1960 [1], foi um dos primeiros passos na simulação de escoamentos incompressíveis com superfícies livres. O método MAC é capaz de simular escoamentos transientes e incompressíveis de fluidos newtonianos em domínios bidimensionais e com geometria simples, utilizando o método de diferenças finitas em uma malha deslocada. A região correspondente ao fluido é descrita por um conjunto de partículas marcadoras que se movimentam de acordo com a velocidade do escoamento na célula computacional que as contém. As variáveis primitivas empregadas na simulação são a pressão e as duas componentes da velocidade. Um dos melhoramentos implementados no 
método MAC é o método SMAC (Simplified-Marker-and-Cell) [2], cuja principal característica é a divisão do ciclo computacional em duas partes: um cálculo prévio da velocidade, seguido de uma correção desta através de uma função potencial, de forma a satisfazer o princípio de conservação de massa. Esta técnica contorna dificuldades de cálculo do método original. Baseados no método SMAC, Tomé e McKee desenvolveram o método GENSMAC (Generalized-Simplified-Marker-and-Cell) [3, 4]. O método GENSMAC é uma técnica numérica para simular escoamentos newtonianos com superfícies livres em domínios bidimensionais com geometria complexa, permitindo a utilização de várias entradas e saídas de fluido e contornos rígidos curvos. Esta técnica foi estendida para fluidos newtonianos generalizados [5].

Visando obter uma técnica numérica para simular escoamentos tridimensionais com superfícies livres, Tomé et al. [6] desenvolveram o método GENSMAC3D, que consiste na extensão do método GENSMAC original para escoamentos cartesianos tridimensionais. O método GENSMAC3D foi implementado computacionalmente em um ambiente de simulação denominado Freeflow3D [7]. O ambiente Freeflow3D é composto por três módulos: Modflow3D - responsável pela modelagem de objetos tais como contêineres e injetores, e especificação do problema (como a especificação do domínio, atribuição das constantes adimensionais, inicialização de variáveis, etc.); Simflow3D a parte central do Freeflow3D, pois este módulo implementa a discretização das equações governantes utilizando o método de diferenças finitas em uma malha tridimensional deslocada; e Visflow3D - que disponibiliza a visualização dos dados gerados pelo módulo Simflow3D utilizando ferramentas de computação gráfica. Detalhes sobre tais módulos podem ser encontrados em Castelo et al. [7].

Basicamente, todas as referências citadas até o presente momento tratam de fluidos newtonianos com exceção de fluidos newtonianos generalizados, onde a viscosidade varia com a taxa de cisalhamento e pode ser modelada, por exemplo, pelos modelos de Cross, Ellis, Power Law ou Carreau-Yasuda. A simulação de escoamentos de fluidos newtonianos generalizados foi o ponto de partida para o tratamento de escoamentos não-newtonianos. Além do mais, ao longo das últimas décadas, diversas indústrias têm substituído ma- 
teriais metálicos por polímeros (fluidos viscoelásticos), devido à maior leveza e flexibilidade, com durabilidade equivalente aos anteriores. Portanto, há uma necessidade crescente de se estudar os fluidos viscoelásticos. Estudos teóricos visando obter equações matemáticas capazes de modelar escoamentos de fluidos viscoelásticos o mais próximo possível da realidade constituem uma linha de pesquisa na área de fluidos viscoelásticos. Além disso, ensaios experimentais do comportamento de fluidos viscoelásticos e estudos de simulação numérica podem ser utilizados para prever o comportamento desses fluidos.

Na literatura é possível encontrar uma vasta quantidade de artigos que tratam de modelos viscoelásticos, tais como:

- modelo de Maxwell (UCM - upper-convected Maxwell) [8, 9]

- modelo Oldroyd-B [9-12]

- modelo PTT (Phan-Thien-Tanner) [12-17]

- modelo K-BKZ (Kaye-Bernstein, Kearsley e Zapas) [18-22]

Dentre os modelos acima citados, os três primeiros são modelados por equações diferenciais parciais, ao passo que o último é modelado por uma equação integral. O modelo de Maxwell (UCM) é um dos mais antigos modelos utilizados para descrever o efeito da viscoelasticidade em um determinado fluido, incorporando características tanto de um sólido elástico Hookeano quanto de um fluido viscoso newtoniano.

Devido à complexidade dos escoamentos viscoelásticos, boa parte dos trabalhos estão relacionados a escoamentos confinados, como o problema da contração [12, 14, 23-25] e a simulação numérica do escoamento totalmente desenvolvido em canais ou tubos $[15,17,26]$, ou a problemas onde a superfície livre apresenta pequenas deformações [10, 27-29]. Recentemente, Tomé et al. [30] apresentaram uma técnica numérica para simular escoamentos viscoelásticos bidimensionais com superfícies livres em movimento, utilizando o modelo Oldroyd-B. Resultados numéricos para o problema do jato oscilante e do inchamento do extrudado foram apresentados para diversos valores do 
número de Weissenberg. Posteriormente, a técnica foi estendida para escoamentos tridimensionais em coordenadas cartesianas [31] e cilíndricas [32]. Concomitantemente, foram incorporados ao ambiente Freeflow3D escoamentos em três dimensões cartesianas do fluido PTT [33, 34]. Neste trabalho, será apresentado o desenvolvimento, a implementação e a validação de um método numérico para simular escoamentos transientes e incompressíveis no espaço cartesiano tridimensional, utilizando o modelo UCM, bem como resultados numéricos para os problemas do jato oscilante e do inchamento do extrudado.

\section{Organização da dissertação}

Este trabalho está organizado da seguinte maneira:

- Capítulo 1: são apresentadas as equações governantes de escoamentos incompressíveis e isotérmicos envolvendo o fluido UCM e a adimensionalização adotada, bem como a formulação EVSS para a resolução do sistema de equações governantes. As condições de contorno para a velocidade e o cálculo do tensor não-newtoniano em contornos rígidos são estudados em detalhes, juntamente com as condições de contorno na superfície livre para a pressão e a velocidade.

- Capítulo 2: o algoritmo numérico é descrito em detalhes.

- Capítulo 3: a discretização das equações utilizando o método de diferenças finitas é apresentada juntamente com a classificação das células computacionais utilizada no algoritmo GENSMAC.

- Capítulo 4: são apresentados os resultados da validação do método numérico utilizando a solução analítica para escoamentos desenvolvidos em um tubo. A convergência é demonstrada através do refinamento de malha computacional.

- Capítulo 5: são apresentados resultados das simulações dos fenômenos do jato oscilante e do inchamento do extrudado. Uma comparação dos 
resultados obtidos com os fluidos UCM e newtoniano é realizada, bem como a comparação com outros trabalhos experimentais, numéricos e teóricos encontrados na literatura.

- Capítulo 6: esse capítulo apresenta as conclusões finais relacionadas ao trabalho desenvolvido, e sugestões de trabalhos futuros utilizando a técnica numérica apresentada. 


\section{Capítulo 1}

\section{Equações governantes}

As equações que governam escoamentos isotérmicos e incompressíveis envolvendo um fluido upper-convected Maxwell são:

- a equação constitutiva

$$
\mathbf{T}+\lambda \stackrel{\nabla}{\mathbf{T}}=2 \mu \mathbf{D}
$$

- a equação de conservação de quantidade de movimento

$$
\frac{\partial \mathbf{u}}{\partial t}+\nabla \cdot(\mathbf{u u})=\frac{1}{\rho}(-\nabla p+\nabla \cdot \mathbf{T})+\mathbf{g}
$$

- e a equação da continuidade

$$
\nabla \cdot \mathbf{u}=0
$$

onde $\mathbf{u}$ denota o vetor velocidade, $\mathbf{T}$ é o tensor extra-tensão, $p$ e $\mathbf{g}$ são, respectivamente, a pressão e o campo gravitacional atuantes sobre o fluido, $\rho$ é a massa específica do fluido e $\mu$ sua viscosidade.

Na equação (1.1), $\lambda$ é uma constante temporal de relaxação do fluido, e D é o tensor taxa de deformação, definido por

$$
\mathbf{D}=\frac{1}{2}\left[(\nabla \mathbf{u})+(\nabla \mathbf{u})^{T}\right]
$$


Ainda em (1.1), $\stackrel{\nabla}{\mathbf{T}}$ representa a derivada contravariante de $\mathbf{T}$ (upperconvected derivative):

$$
\stackrel{\nabla}{\mathbf{T}}=\frac{\partial \mathbf{T}}{\partial t}+\nabla \cdot(\mathbf{u} \mathbf{T})-(\nabla \mathbf{u})^{T} \cdot \mathbf{T}-\mathbf{T} \cdot(\nabla \mathbf{u})
$$

Deste ponto em diante, nos referenciaremos ao modelo upper-convected Maxwell simplesmente como modelo ou fluido de Maxwell.

Para resolver o sistema de equações (1.1)-(1.3), vamos adotar a formulação EVSS - elastic-viscous split stress - introduzida por Rajagopalan et al. [35], dada pela decomposição

$$
\mathbf{T}=\mathbf{S}+2 \mu \mathbf{D}
$$

O emprego dessa formulação permite o aparecimento do laplaciano da velocidade na equação de conservação de quantidade de movimento.

Introduzindo-se (1.6) nas equações (1.1) e (1.2), obtêm-se, respectivamente,

$$
\begin{gathered}
\mathbf{S}+\lambda \stackrel{\nabla}{\mathbf{S}}=-2 \lambda \mu \stackrel{\nabla}{\mathbf{D}}, \\
\rho\left[\frac{\partial \mathbf{u}}{\partial t}+\nabla \cdot(\mathbf{u u})\right]=-\nabla p+2 \mu \nabla \cdot \mathbf{D}+\nabla \cdot \mathbf{S}+\rho \mathbf{g} .
\end{gathered}
$$

Observamos que fazendo $\mathbf{S} \equiv \mathbf{0}$ nas equações (1.6) e (1.8) obtemos as equações de Navier-Stokes, que modelam escoamentos de fluidos newtonianos (veja, por exemplo, Chorin [36]).

Portanto as equações governantes utilizadas neste trabalho são a equação da continuidade (1.3), a equação de conservação de quantidade de movimento (1.8) e a equação constitutiva (1.7). 


\subsection{Forma cartesiana tridimensional}

Nesse trabalho vamos considerar escoamentos transientes no espaço cartesiano tridimensional e utilizaremos a seguinte notação:

$$
\mathbf{x}=(x, y, z)^{T}, \quad \mathbf{u}=(u, v, w)^{T}, \quad \mathbf{g}=\left(g_{x}, g_{y}, g_{z}\right)^{T}, \quad \mathbf{S}=\left(\begin{array}{ccc}
S^{x x} & S^{x y} & S^{x z} \\
S^{x y} & S^{y y} & S^{y z} \\
S^{x z} & S^{y z} & S^{z z}
\end{array}\right)
$$

Logo, a equação da conservação de massa (1.3) é escrita como

$$
\frac{\partial u}{\partial x}+\frac{\partial v}{\partial y}+\frac{\partial w}{\partial z}=0
$$

e pode-se mostrar que (veja equação (1.4))

$$
\nabla \cdot \mathbf{D}=\frac{1}{2}\left(\nabla \cdot \nabla \mathbf{u}+\nabla \cdot(\nabla \mathbf{u})^{T}\right)=\frac{1}{2} \nabla^{2} \mathbf{u} .
$$

Substituindo a equação (1.10) em (1.8) e dividindo-se ambos os lados por $\rho$, obtém-se, na forma cartesiana tridimensional,

$$
\begin{aligned}
& \frac{\partial u}{\partial t}+\frac{\partial\left(u^{2}\right)}{\partial x}+\frac{\partial(v u)}{\partial y}+\frac{\partial(w u)}{\partial z}=-\frac{1}{\rho} \frac{\partial p}{\partial x} \\
&+\nu\left(\frac{\partial^{2} u}{\partial x^{2}}+\frac{\partial^{2} u}{\partial y^{2}}+\frac{\partial^{2} u}{\partial z^{2}}\right)+\frac{1}{\rho}\left(\frac{\partial S^{x x}}{\partial x}+\frac{\partial S^{x y}}{\partial y}+\frac{\partial S^{x z}}{\partial z}\right)+g_{x} \\
& \frac{\partial v}{\partial t}+\frac{\partial(u v)}{\partial x}+\frac{\partial\left(v^{2}\right)}{\partial y}+\frac{\partial(w v)}{\partial z}=-\frac{1}{\rho} \frac{\partial p}{\partial y} \\
&+\nu\left(\frac{\partial^{2} v}{\partial x^{2}}+\frac{\partial^{2} v}{\partial y^{2}}+\frac{\partial^{2} v}{\partial z^{2}}\right)+\frac{1}{\rho}\left(\frac{\partial S^{x y}}{\partial x}+\frac{\partial S^{y y}}{\partial y}+\frac{\partial S^{y z}}{\partial z}\right)+g_{y} \\
& \frac{\partial w}{\partial t}+\frac{\partial(u w)}{\partial x}+\frac{\partial(v w)}{\partial y}+\frac{\partial\left(w^{2}\right)}{\partial z}=-\frac{1}{\rho} \frac{\partial p}{\partial z} \\
&+\nu\left(\frac{\partial^{2} w}{\partial x^{2}}+\frac{\partial^{2} w}{\partial y^{2}}+\frac{\partial^{2} w}{\partial z^{2}}\right)+\frac{1}{\rho}\left(\frac{\partial S^{x z}}{\partial x}+\frac{\partial S^{y z}}{\partial y}+\frac{\partial S^{z z}}{\partial z}\right)+g_{z}
\end{aligned}
$$

onde $\nu \equiv \mu / \rho$ é a viscosidade cinemática do fluido.

Expandindo-se as derivadas contravariantes na equação constitutiva (1.7), 
obtém-se

$$
\begin{aligned}
& \mathbf{S}+\lambda\left[\frac{\partial \mathbf{S}}{\partial t}+\nabla \cdot(\mathbf{u S})-(\nabla \mathbf{u})^{T} \cdot \mathbf{S}-\mathbf{S} \cdot(\nabla \mathbf{u})\right]= \\
& -2 \lambda \mu\left[\frac{\partial \mathbf{D}}{\partial t}+\nabla \cdot(\mathbf{u D})-(\nabla \mathbf{u})^{T} \cdot \mathbf{D}-\mathbf{D} \cdot(\nabla \mathbf{u})\right]
\end{aligned}
$$

Considerando-se escoamentos cartesianos tridimensionais, a equação (1.14) pode ser escrita como:

$$
\begin{aligned}
& S^{x x}+\lambda {\left[\frac{\partial S^{x x}}{\partial t}+\frac{\partial\left(u S^{x x}\right)}{\partial x}+\frac{\partial\left(v S^{x x}\right)}{\partial y}+\frac{\partial\left(w S^{x x}\right)}{\partial z}\right.} \\
&-2\left.\left(\frac{\partial u}{\partial x} S^{x x}+\frac{\partial u}{\partial y} S^{x y}+\frac{\partial u}{\partial z} S^{x z}\right)\right]= \\
&-2 \lambda \mu\left[\frac{\partial D^{x x}}{\partial t}+\frac{\partial\left(u D^{x x}\right)}{\partial x}+\frac{\partial\left(v D^{x x}\right)}{\partial y}+\frac{\partial\left(w D^{x x}\right)}{\partial z}\right. \\
&\left.-2\left(\frac{\partial u}{\partial x} D^{x x}+\frac{\partial u}{\partial y} D^{x y}+\frac{\partial u}{\partial z} D^{x z}\right)\right], \\
& S^{y y}+\lambda\left[\frac{\partial S^{y y}}{\partial t}+\frac{\partial\left(u S^{y y}\right)}{\partial x}+\frac{\partial\left(v S^{y y}\right)}{\partial y}+\frac{\partial\left(w S^{y y}\right)}{\partial z}\right. \\
&\left.-2\left(\frac{\partial v}{\partial x} S^{x y}+\frac{\partial v}{\partial y} S^{y y}+\frac{\partial v}{\partial z} S^{y z}\right)\right]= \\
&-2 \lambda \mu\left[\frac{\partial D^{y y}}{\partial t}+\frac{\partial\left(u D^{y y}\right)}{\partial x}+\frac{\partial\left(v D^{y y}\right)}{\partial y}+\frac{\partial\left(w D^{y y}\right)}{\partial z}\right. \\
&\left.-2\left(\frac{\partial v}{\partial x} D^{x y}+\frac{\partial v}{\partial y} D^{y y}+\frac{\partial v}{\partial z} D^{y z}\right)\right] \\
&\left.-2\left(\frac{\partial w}{\partial x} D^{x z}+\frac{\partial w}{\partial y} D^{y z}+\frac{\partial w}{\partial z} D^{z z}\right)\right] \\
& S^{z z}+\lambda\left[\frac{\partial S^{z z}}{\partial t}+\frac{\partial\left(u S^{z z}\right)}{\partial x}+\frac{\partial\left(v S^{z z}\right)}{\partial y}+\frac{\partial\left(w S^{z z}\right)}{\partial z}\right. \\
&\left.-2\left(\frac{\partial w}{\partial x} S^{x z}+\frac{\partial w}{\partial y} S^{y z}+\frac{\partial w}{\partial z} S^{z z}\right)\right]= \\
&-2 \lambda \mu\left[\frac{\partial D^{z z}}{\partial t}+\frac{\partial\left(u D^{z z}\right)}{\partial x}+\frac{\partial\left(v D^{z z}\right)}{\partial y}+\frac{\partial\left(w D^{z z}\right)}{\partial z}\right. \\
&-
\end{aligned}
$$




$$
\begin{aligned}
S^{x y} & +\lambda\left[\frac{\partial S^{x y}}{\partial t}+\frac{\partial\left(u S^{x y}\right)}{\partial x}+\frac{\partial\left(v S^{x y}\right)}{\partial y}+\frac{\partial\left(w S^{x y}\right)}{\partial z}\right. \\
& \left.-\frac{\partial u}{\partial x} S^{x y}-\frac{\partial u}{\partial y} S^{y y}-\frac{\partial u}{\partial z} S^{y z}-\frac{\partial v}{\partial x} S^{x x}-\frac{\partial v}{\partial y} S^{x y}-\frac{\partial v}{\partial z} S^{x z}\right]= \\
& -2 \lambda \mu\left[\frac{\partial D^{x y}}{\partial t}+\frac{\partial\left(u D^{x y}\right)}{\partial x}+\frac{\partial\left(v D^{x y}\right)}{\partial y}+\frac{\partial\left(w D^{x y}\right)}{\partial z}\right. \\
& \left.-\frac{\partial u}{\partial x} D^{x y}-\frac{\partial u}{\partial y} D^{y y}-\frac{\partial u}{\partial z} D^{y z}-\frac{\partial v}{\partial x} D^{x x}-\frac{\partial v}{\partial y} D^{x y}-\frac{\partial v}{\partial z} D^{x z}\right] \\
S^{x z}+ & \lambda\left[\frac{\partial S^{x z}}{\partial t}+\frac{\partial\left(u S^{x z}\right)}{\partial x}+\frac{\partial\left(v S^{x z}\right)}{\partial y}+\frac{\partial\left(w S^{x z}\right)}{\partial z}\right. \\
- & \left.\frac{\partial u}{\partial x} S^{x z}-\frac{\partial u}{\partial y} S^{y z}-\frac{\partial u}{\partial z} S^{z z}-\frac{\partial w}{\partial x} S^{x x}-\frac{\partial w}{\partial y} S^{x y}-\frac{\partial w}{\partial z} S^{x z}\right]= \\
- & 2 \lambda \mu\left[\frac{\partial D^{x z}}{\partial t}+\frac{\partial\left(u D^{x z}\right)}{\partial x}+\frac{\partial\left(v D^{x z}\right)}{\partial y}+\frac{\partial\left(w D^{x z}\right)}{\partial z}\right. \\
- & \left.\frac{\partial u}{\partial x} D^{x z}-\frac{\partial u}{\partial y} D^{y z}-\frac{\partial u}{\partial z} D^{z z}-\frac{\partial w}{\partial x} D^{x x}-\frac{\partial w}{\partial y} D^{x y}-\frac{\partial w}{\partial z} D^{x z}\right] \\
- & \left.\frac{\partial v}{\partial x} D^{x z}-\frac{\partial v}{\partial y} D^{y z}-\frac{\partial v}{\partial z} D^{z z}-\frac{\partial w}{\partial x} D^{x y}-\frac{\partial w}{\partial y} D^{y y}-\frac{\partial w}{\partial z} D^{y z}\right] \\
- & \left.\frac{\partial v}{\partial x} S^{x z}-\frac{\partial v}{\partial y} S^{y z}-\frac{\partial v}{\partial z} S^{z z}-\frac{\partial w}{\partial x} S^{x y}-\frac{\partial w}{\partial y} S^{y y}-\frac{\partial w}{\partial z} S^{y z}\right]= \\
S^{y z}+ & \lambda\left[\frac{\partial S^{y z}}{\partial t}+\frac{\partial\left(u D^{y z}\right)}{\partial t}+\frac{\partial\left(v D^{y z}\right)}{\partial y}+\frac{\partial\left(w D^{y z}\right)}{\partial z}\right. \\
& =
\end{aligned}
$$

\subsection{Adimensionalização}

Introduzindo-se as variáveis adimensionais

$$
\overline{\mathbf{x}}=\frac{1}{L} \mathbf{x}, \quad \bar{t}=\frac{U}{L} t, \quad \overline{\mathbf{u}}=\frac{1}{U} \mathbf{u}, \quad \bar{p}=\frac{1}{\rho U^{2}} p, \quad \overline{\mathbf{g}}=\frac{1}{g} \mathbf{g}, \quad \overline{\mathbf{S}}=\frac{1}{\rho U^{2}} \mathbf{S},
$$


em que $U$ e $L$ são respectivamente, velocidade e comprimento característicos do problema em estudo, e $g$ é a constante gravitacional, a equação da continuidade (1.9), as componentes da equação de conservação de quantidade de movimento (1.11)-(1.13) e as componentes da equação constitutiva de Maxwell (1.15)-(1.20) são reescritas, respectivamente, como

$$
\begin{aligned}
& \frac{\partial u}{\partial x}+\frac{\partial v}{\partial y}+\frac{\partial w}{\partial z}=0 \\
& \frac{\partial u}{\partial t}+\frac{\partial\left(u^{2}\right)}{\partial x}+\frac{\partial(v u)}{\partial y}+\frac{\partial(w u)}{\partial z}=-\frac{\partial p}{\partial x} \\
& +\frac{1}{R e}\left(\frac{\partial^{2} u}{\partial x^{2}}+\frac{\partial^{2} u}{\partial y^{2}}+\frac{\partial^{2} u}{\partial z^{2}}\right)+\frac{\partial S^{x x}}{\partial x}+\frac{\partial S^{x y}}{\partial y}+\frac{\partial S^{x z}}{\partial z}+\frac{1}{F r^{2}} g_{x}, \\
& \frac{\partial v}{\partial t}+\frac{\partial(u v)}{\partial x}+\frac{\partial\left(v^{2}\right)}{\partial y}+\frac{\partial(w v)}{\partial z}=-\frac{\partial p}{\partial y} \\
& +\frac{1}{R e}\left(\frac{\partial^{2} v}{\partial x^{2}}+\frac{\partial^{2} v}{\partial y^{2}}+\frac{\partial^{2} v}{\partial z^{2}}\right)+\frac{\partial S^{x y}}{\partial x}+\frac{\partial S^{y y}}{\partial y}+\frac{\partial S^{y z}}{\partial z}+\frac{1}{F r^{2}} g_{y}, \\
& \frac{\partial w}{\partial t}+\frac{\partial(u w)}{\partial x}+\frac{\partial(v w)}{\partial y}+\frac{\partial\left(w^{2}\right)}{\partial z}=-\frac{\partial p}{\partial z} \\
& +\frac{1}{R e}\left(\frac{\partial^{2} w}{\partial x^{2}}+\frac{\partial^{2} w}{\partial y^{2}}+\frac{\partial^{2} w}{\partial z^{2}}\right)+\frac{\partial S^{x z}}{\partial x}+\frac{\partial S^{y z}}{\partial y}+\frac{\partial S^{z z}}{\partial z}+\frac{1}{F r^{2}} g_{z}, \\
& S^{x x}+W e\left[\frac{\partial S^{x x}}{\partial t}+\frac{\partial\left(u S^{x x}\right)}{\partial x}+\frac{\partial\left(v S^{x x}\right)}{\partial y}+\frac{\partial\left(w S^{x x}\right)}{\partial z}\right. \\
& \left.-2\left(\frac{\partial u}{\partial x} S^{x x}+\frac{\partial u}{\partial y} S^{x y}+\frac{\partial u}{\partial z} S^{x z}\right)\right]= \\
& -2 \frac{W e}{R e}\left[\frac{\partial D^{x x}}{\partial t}+\frac{\partial\left(u D^{x x}\right)}{\partial x}+\frac{\partial\left(v D^{x x}\right)}{\partial y}+\frac{\partial\left(w D^{x x}\right)}{\partial z}\right. \\
& \left.-2\left(\frac{\partial u}{\partial x} D^{x x}+\frac{\partial u}{\partial y} D^{x y}+\frac{\partial u}{\partial z} D^{x z}\right)\right],
\end{aligned}
$$




$$
\begin{aligned}
& S^{y y}+W e\left[\frac{\partial S^{y y}}{\partial t}+\frac{\partial\left(u S^{y y}\right)}{\partial x}+\frac{\partial\left(v S^{y y}\right)}{\partial y}+\frac{\partial\left(w S^{y y}\right)}{\partial z}\right. \\
& \left.-2\left(\frac{\partial v}{\partial x} S^{x y}+\frac{\partial v}{\partial y} S^{y y}+\frac{\partial v}{\partial z} S^{y z}\right)\right]= \\
& -2 \frac{W e}{R e}\left[\frac{\partial D^{y y}}{\partial t}+\frac{\partial\left(u D^{y y}\right)}{\partial x}+\frac{\partial\left(v D^{y y}\right)}{\partial y}+\frac{\partial\left(w D^{y y}\right)}{\partial z}\right. \\
& \left.-2\left(\frac{\partial v}{\partial x} D^{x y}+\frac{\partial v}{\partial y} D^{y y}+\frac{\partial v}{\partial z} D^{y z}\right)\right] \\
& S^{z z}+W e\left[\frac{\partial S^{z z}}{\partial t}+\frac{\partial\left(u S^{z z}\right)}{\partial x}+\frac{\partial\left(v S^{z z}\right)}{\partial y}+\frac{\partial\left(w S^{z z}\right)}{\partial z}\right. \\
& \left.-2\left(\frac{\partial w}{\partial x} S^{x z}+\frac{\partial w}{\partial y} S^{y z}+\frac{\partial w}{\partial z} S^{z z}\right)\right]= \\
& -2 \frac{W e}{R e}\left[\frac{\partial D^{z z}}{\partial t}+\frac{\partial\left(u D^{z z}\right)}{\partial x}+\frac{\partial\left(v D^{z z}\right)}{\partial y}+\frac{\partial\left(w D^{z z}\right)}{\partial z}\right. \\
& \left.-2\left(\frac{\partial w}{\partial x} D^{x z}+\frac{\partial w}{\partial y} D^{y z}+\frac{\partial w}{\partial z} D^{z z}\right)\right] \\
& S^{x y}+W e\left[\frac{\partial S^{x y}}{\partial t}+\frac{\partial\left(u S^{x y}\right)}{\partial x}+\frac{\partial\left(v S^{x y}\right)}{\partial y}+\frac{\partial\left(w S^{x y}\right)}{\partial z}\right. \\
& \left.-\frac{\partial u}{\partial x} S^{x y}-\frac{\partial u}{\partial y} S^{y y}-\frac{\partial u}{\partial z} S^{y z}-\frac{\partial v}{\partial x} S^{x x}-\frac{\partial v}{\partial y} S^{x y}-\frac{\partial v}{\partial z} S^{x z}\right]= \\
& -2 \frac{W e}{R e}\left[\frac{\partial D^{x y}}{\partial t}+\frac{\partial\left(u D^{x y}\right)}{\partial x}+\frac{\partial\left(v D^{x y}\right)}{\partial y}+\frac{\partial\left(w D^{x y}\right)}{\partial z}\right. \\
& \left.-\frac{\partial u}{\partial x} D^{x y}-\frac{\partial u}{\partial y} D^{y y}-\frac{\partial u}{\partial z} D^{y z}-\frac{\partial v}{\partial x} D^{x x}-\frac{\partial v}{\partial y} D^{x y}-\frac{\partial v}{\partial z} D^{x z}\right], \\
& S^{x z}+W e\left[\frac{\partial S^{x z}}{\partial t}+\frac{\partial\left(u S^{x z}\right)}{\partial x}+\frac{\partial\left(v S^{x z}\right)}{\partial y}+\frac{\partial\left(w S^{x z}\right)}{\partial z}\right. \\
& \left.-\frac{\partial u}{\partial x} S^{x z}-\frac{\partial u}{\partial y} S^{y z}-\frac{\partial u}{\partial z} S^{z z}-\frac{\partial w}{\partial x} S^{x x}-\frac{\partial w}{\partial y} S^{x y}-\frac{\partial w}{\partial z} S^{x z}\right]= \\
& -2 \frac{W e}{R e}\left[\frac{\partial D^{x z}}{\partial t}+\frac{\partial\left(u D^{x z}\right)}{\partial x}+\frac{\partial\left(v D^{x z}\right)}{\partial y}+\frac{\partial\left(w D^{x z}\right)}{\partial z}\right. \\
& \left.-\frac{\partial u}{\partial x} D^{x z}-\frac{\partial u}{\partial y} D^{y z}-\frac{\partial u}{\partial z} D^{z z}-\frac{\partial w}{\partial x} D^{x x}-\frac{\partial w}{\partial y} D^{x y}-\frac{\partial w}{\partial z} D^{x z}\right],
\end{aligned}
$$




$$
\begin{aligned}
S^{y z} & +W e\left[\frac{\partial S^{y z}}{\partial t}+\frac{\partial\left(u S^{y z}\right)}{\partial x}+\frac{\partial\left(v S^{y z}\right)}{\partial y}+\frac{\partial\left(w S^{y z}\right)}{\partial z}\right. \\
& \left.-\frac{\partial v}{\partial x} S^{x z}-\frac{\partial v}{\partial y} S^{y z}-\frac{\partial v}{\partial z} S^{z z}-\frac{\partial w}{\partial x} S^{x y}-\frac{\partial w}{\partial y} S^{y y}-\frac{\partial w}{\partial z} S^{y z}\right]= \\
& -2 \frac{W e}{R e}\left[\frac{\partial D^{y z}}{\partial t}+\frac{\partial\left(u D^{y z}\right)}{\partial x}+\frac{\partial\left(v D^{y z}\right)}{\partial y}+\frac{\partial\left(w D^{y z}\right)}{\partial z}\right. \\
& \left.-\frac{\partial v}{\partial x} D^{x z}-\frac{\partial v}{\partial y} D^{y z}-\frac{\partial v}{\partial z} D^{z z}-\frac{\partial w}{\partial x} D^{x y}-\frac{\partial w}{\partial y} D^{y y}-\frac{\partial w}{\partial z} D^{y z}\right]
\end{aligned}
$$

Nas equações (1.21)-(1.30), os números adimensionais $R e=\frac{\rho U L}{\mu}=\frac{U L}{\nu}$, $F r=\frac{U}{\sqrt{g L}}$ e $W e=\frac{\lambda U}{L}$ são, respectivamente, o número de Reynolds, o número de Froude e o número de Weissenberg. Para simplificação da notação, nas equações acima e deste ponto em diante as barras das variáveis adimensionais são omitidas. O número de Reynolds define a relação entre as forças inerciais e viscosas de um escoamento, permitindo a identificação do regime de escoamento (laminar ou turbulento). Neste projeto, são utilizados valores do número de Reynolds da ordem da unidade, de maneira que o regime em estudo é sempre laminar. O número de Weissenberg por sua vez relaciona o tempo de relaxação do fluido a um tempo específico do processo, de forma a caracterizar a resposta do fluido às tensões a ele impostas. Quanto maior o número de Weissenberg, maior a elasticidade do material. São adotados valores do número de Weissenberg entre 0 e 0.5 neste trabalho. O número de Froude por sua vez relaciona as forças inerciais e gravitacionais do escoamento, e não será foco de nossas atenções neste texto. 


\subsection{Condições de contorno}

Parte-se de condições iniciais definidas em um instante $t_{0}$ para $\mathbf{u}$ e $\mathbf{S}$, dadas por

$$
\begin{aligned}
& \mathbf{u}\left(x, t_{0}\right)=\mathbf{0}, \\
& \mathbf{S}\left(x, t_{0}\right)=\mathbf{0} .
\end{aligned}
$$

Para a velocidade, adotam-se as seguintes condições de contorno:

- Em contornos rígidos, utiliza-se a condição de Dirichlet nula

$$
\mathbf{u}_{n}=0, \quad \mathbf{u}_{t_{1}}=0, \quad \mathbf{u}_{t_{2}}=0
$$

- em entradas de fluido (inflows),

$$
\mathbf{u}_{n}=f(U), \quad \mathbf{u}_{t_{1}}=0, \quad \mathbf{u}_{t_{2}}=0
$$

- e em saídas de fluido (outflows),

$$
\frac{\partial \mathbf{u}_{n}}{\partial n}=0, \quad \frac{\partial \mathbf{u}_{t_{1}}}{\partial n}=0, \quad \frac{\partial \mathbf{u}_{t_{2}}}{\partial n}=0
$$

onde $n$ e $t_{1}$ e $t_{2}$ denotam, respectivamente, direções normais e tangenciais aos contornos rígidos, entradas e saídas de fluido. No caso de contornos rígidos, $\mathbf{u}_{n}=0$ define a condição de impermeabilidade na parede descrita pelo contorno, ao passo que $\mathbf{u}_{t_{1}}=0$ e $\mathbf{u}_{t_{2}}=0$ definem as condições de nãoescorregamento (no-slip) da mesma. $f(U)$ é uma função quadrática de $U$, que gera um perfil parabólico ou linear.

No caso do tensor não-newtoniano, as condições de contorno adotadas são as seguintes:

- Entradas de fluido: Assumem-se que não existem perturbações em tais regiões, de modo que as tensões sejam nulas. Dessa forma, de acordo 
com Crochet et al. [37] e Mompean e Deville [9], temos que:

$$
S^{x x}=S^{y y}=S^{z z}=S^{x y}=S^{x z}=S^{y z}=0 .
$$

- Saídas de fluido: Utilizam-se condições de Neumann nulas, como especificado para a velocidade $\mathbf{u}$ :

$$
\frac{\partial S^{x x}}{\partial n}=\frac{\partial S^{y y}}{\partial n}=\frac{\partial S^{z z}}{\partial n}=\frac{\partial S^{x y}}{\partial n}=\frac{\partial S^{x z}}{\partial n}=\frac{\partial S^{y z}}{\partial n}=0,
$$

onde $n$ denota a direção normal ao plano da saída de fluido.

No caso de contornos rígidos, as componentes do tensor não-newtoniano são obtidas conforme descrito na Seção 1.4 a seguir.

\subsection{Cálculo do tensor $\mathrm{S}$ em contornos rígidos}

Para calcular as componentes do tensor não-newtoniano $\mathbf{S}$, utilizaremos um método upwind de alta ordem denominado CUBISTA (Convergent and Universally Bounded Interpolation Scheme for the Treatment of Advection), desenvolvido por Alves et al. [38], para aproximar os termos convectivos. Este método necessita que os valores das variáveis sejam definidos em até duas células vizinhas. Logo, para cálculos nos pontos próximos aos contornos rígidos há a necessidade de se conhecer o valor do tensor não-newtoniano $\mathbf{S}$ em tais contornos.

Os valores das componentes do tensor $\mathbf{S}$ no contorno rígido são obtidos através das equações (1.25)-(1.30), assumidas válidas juntamente com a condição inicial $\mathbf{S}=\mathbf{0}$.

Inicialmente, visando eliminar os termos lineares em $\mathbf{S}$, introduz-se a mudança de variáveis (ver Tomé et al. [30])

$$
\mathbf{S}=e^{-\frac{1}{W e} t} \widetilde{\mathbf{S}}
$$


nas equações (1.25)-(1.30), o que resulta em

$$
\begin{aligned}
& \frac{\partial \widetilde{S}^{x x}}{\partial t}=-\frac{\partial\left(u \widetilde{S}^{x x}\right)}{\partial x}-\frac{\partial\left(v \widetilde{S}^{x x}\right)}{\partial y}-\frac{\partial\left(w \widetilde{S}^{x x}\right)}{\partial z} \\
& +2\left(\frac{\partial u}{\partial x} \widetilde{S}^{x x}+\frac{\partial u}{\partial y} \widetilde{S}^{x y}+\frac{\partial u}{\partial z} \widetilde{S}^{x z}\right) \\
& -\frac{2}{R e} e^{\frac{1}{W e} t}\left[\frac{\partial D^{x x}}{\partial t}+\frac{\partial\left(u D^{x x}\right)}{\partial x}+\frac{\partial\left(v D^{x x}\right)}{\partial y}+\frac{\partial\left(w D^{x x}\right)}{\partial z}\right. \\
& \left.-2\left(\frac{\partial u}{\partial x} D^{x x}+\frac{\partial u}{\partial y} D^{x y}+\frac{\partial u}{\partial z} D^{x z}\right)\right]
\end{aligned}
$$

$$
\begin{aligned}
& \frac{\partial \widetilde{S}^{y y}}{\partial t}=-\frac{\partial\left(u \widetilde{S}^{y y}\right)}{\partial x}-\frac{\partial\left(v \widetilde{S}^{y y}\right)}{\partial y}-\frac{\partial\left(w \widetilde{S}^{y y}\right)}{\partial z} \\
& +2\left(\frac{\partial v}{\partial x} \widetilde{S}^{x y}+\frac{\partial v}{\partial y} \widetilde{S}^{y y}+\frac{\partial v}{\partial z} \widetilde{S}^{y z}\right) \\
& -\frac{2}{R e} e^{\frac{1}{W e} t}\left[\frac{\partial D^{y y}}{\partial t}+\frac{\partial\left(u D^{y y}\right)}{\partial x}+\frac{\partial\left(v D^{y y}\right)}{\partial y}+\frac{\partial\left(w D^{y y}\right)}{\partial z}\right. \\
& \left.-2\left(\frac{\partial v}{\partial x} D^{x y}+\frac{\partial v}{\partial y} D^{y y}+\frac{\partial v}{\partial z} D^{y z}\right)\right]
\end{aligned}
$$

$$
\begin{aligned}
& \frac{\partial \widetilde{S}^{z z}}{\partial t}=-\frac{\partial\left(u \widetilde{S}^{z z}\right)}{\partial x}-\frac{\partial\left(v \widetilde{S}^{z z}\right)}{\partial y}-\frac{\partial\left(w \widetilde{S}^{z z}\right)}{\partial z} \\
& +2\left(\frac{\partial w}{\partial x} \widetilde{S}^{x z}+\frac{\partial w}{\partial y} \widetilde{S}^{y z}+\frac{\partial w}{\partial z} \widetilde{S}^{z z}\right) \\
& -\frac{2}{R e} e^{\frac{1}{W e} t}\left[\frac{\partial D^{z z}}{\partial t}+\frac{\partial\left(u D^{z z}\right)}{\partial x}+\frac{\partial\left(v D^{z z}\right)}{\partial y}+\frac{\partial\left(w D^{z z}\right)}{\partial z}\right. \\
& \left.-2\left(\frac{\partial w}{\partial x} D^{x z}+\frac{\partial w}{\partial y} D^{y z}+\frac{\partial w}{\partial z} D^{z z}\right)\right]
\end{aligned}
$$




$$
\begin{aligned}
& \frac{\partial \widetilde{S}^{x y}}{\partial t}=-\frac{\partial\left(u \widetilde{S}^{x y}\right)}{\partial x}-\frac{\partial\left(v \widetilde{S}^{x y}\right)}{\partial y}-\frac{\partial\left(w \widetilde{S}^{x y}\right)}{\partial z} \\
& +\frac{\partial u}{\partial x} \widetilde{S}^{x y}+\frac{\partial u}{\partial y} \widetilde{S}^{y y}+\frac{\partial u}{\partial z} \widetilde{S}^{y z}+\frac{\partial v}{\partial x} \widetilde{S}^{x x}+\frac{\partial v}{\partial y} \widetilde{S}^{x y}+\frac{\partial v}{\partial z} \widetilde{S}^{x z} \\
& -\frac{2}{R e} e^{\frac{1}{W e} t}\left[\frac{\partial D^{x y}}{\partial t}+\frac{\partial\left(u D^{x y}\right)}{\partial x}+\frac{\partial\left(v D^{x y}\right)}{\partial y}+\frac{\partial\left(w D^{x y}\right)}{\partial z}\right. \\
& \left.-\frac{\partial u}{\partial x} D^{x y}-\frac{\partial u}{\partial y} D^{y y}-\frac{\partial u}{\partial z} D^{y z}-\frac{\partial v}{\partial x} D^{x x}-\frac{\partial v}{\partial y} D^{x y}-\frac{\partial v}{\partial z} D^{x z}\right], \\
& \frac{\partial \widetilde{S}^{x z}}{\partial t}=-\frac{\partial\left(u \widetilde{S}^{x z}\right)}{\partial x}-\frac{\partial\left(v \widetilde{S}^{x z}\right)}{\partial y}-\frac{\partial\left(w \widetilde{S}^{x z}\right)}{\partial z} \\
& +\frac{\partial u}{\partial x} \widetilde{S}^{x z}+\frac{\partial u}{\partial y} \widetilde{S}^{y z}+\frac{\partial u}{\partial z} \widetilde{S}^{z z}+\frac{\partial w}{\partial x} \widetilde{S}^{x x}+\frac{\partial w}{\partial y} \widetilde{S}^{x y}+\frac{\partial w}{\partial z} \widetilde{S}^{x z} \\
& -\frac{2}{R e} e^{\frac{1}{W e} t}\left[\frac{\partial D^{x z}}{\partial t}+\frac{\partial\left(u D^{x z}\right)}{\partial x}+\frac{\partial\left(v D^{x z}\right)}{\partial y}+\frac{\partial\left(w D^{x z}\right)}{\partial z}\right. \\
& \left.-\frac{\partial u}{\partial x} D^{x z}-\frac{\partial u}{\partial y} D^{y z}-\frac{\partial u}{\partial z} D^{z z}-\frac{\partial w}{\partial x} D^{x x}-\frac{\partial w}{\partial y} D^{x y}-\frac{\partial w}{\partial z} D^{x z}\right], \\
& \frac{\partial \widetilde{S}^{y z}}{\partial t}=-\frac{\partial\left(u \widetilde{S}^{y z}\right)}{\partial x}-\frac{\partial\left(v \widetilde{S}^{y z}\right)}{\partial y}-\frac{\partial\left(w \widetilde{S}^{y z}\right)}{\partial z} \\
& +\frac{\partial v}{\partial x} \widetilde{S}^{x z}+\frac{\partial v}{\partial y} \widetilde{S}^{y z}+\frac{\partial v}{\partial z} \widetilde{S}^{z z}+\frac{\partial w}{\partial x} \widetilde{S}^{x y}+\frac{\partial w}{\partial y} \widetilde{S}^{y y}+\frac{\partial w}{\partial z} \widetilde{S}^{y z} \\
& -\frac{2}{R e} e^{\frac{1}{W e} t}\left[\frac{\partial D^{y z}}{\partial t}+\frac{\partial\left(u D^{y z}\right)}{\partial x}+\frac{\partial\left(v D^{y z}\right)}{\partial y}+\frac{\partial\left(w D^{y z}\right)}{\partial z}\right. \\
& \left.-\frac{\partial v}{\partial x} D^{x z}-\frac{\partial v}{\partial y} D^{y z}-\frac{\partial v}{\partial z} D^{z z}-\frac{\partial w}{\partial x} D^{x y}-\frac{\partial w}{\partial y} D^{y y}-\frac{\partial w}{\partial z} D^{y z}\right] .
\end{aligned}
$$

As equações (1.39)-(1.44) são resolvidas considerando-se contornos rígidos estacionários paralelos aos planos $x y, x z$ e $y z$, como segue:

\subsubsection{Contornos rígidos paralelos ao plano $x y$}

Em tais contornos, as condições de não-escorregamento e impermeabilidade fornecem $u=v=w=0$ e portanto, $\frac{\partial u}{\partial x}=\frac{\partial v}{\partial x}=\frac{\partial w}{\partial x}=0, \frac{\partial u}{\partial y}=\frac{\partial v}{\partial y}=\frac{\partial w}{\partial y}=0$, e pela equação da continuidade (1.21), $\frac{\partial w}{\partial z}=0$. Dessa forma, $D^{x x}=D^{x y}=$ 


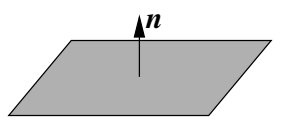

(a)
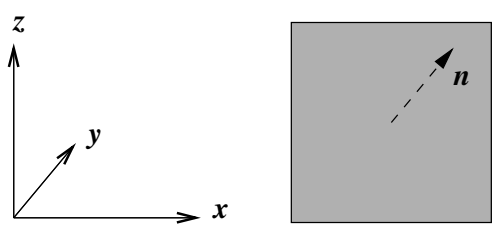

(b)

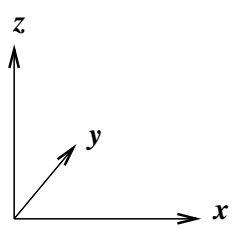

(c)

Figura 1.1: Contornos rígidos paralelos ao plano $x y$ (a), $x z$ (b) e $y z$ (c).

$D^{y y}=D^{z z}=0, D^{x z}=\frac{1}{2} \frac{\partial u}{\partial z}$ e $D^{y z}=\frac{1}{2} \frac{\partial v}{\partial z}$. Neste caso, as equações (1.39)(1.44) se reduzem a

$$
\begin{gathered}
\frac{\partial \widetilde{S}^{x x}}{\partial t}=2 \frac{\partial u}{\partial z} \widetilde{S}^{x z}+\frac{2}{R e} e^{\frac{1}{W e} t}\left(\frac{\partial u}{\partial z}\right)^{2}, \\
\frac{\partial \widetilde{S}^{y y}}{\partial t}=2 \frac{\partial v}{\partial z} \widetilde{S}^{y z}+\frac{2}{R e} e^{\frac{1}{W e} t}\left(\frac{\partial v}{\partial z}\right)^{2}, \\
\frac{\partial \widetilde{S}^{z z}}{\partial t}=0, \\
\frac{\partial \widetilde{S}^{x y}}{\partial t}=\frac{\partial u}{\partial z} \widetilde{S}^{y z}+\frac{\partial v}{\partial z} \widetilde{S}^{x z}+\frac{2}{R e} e^{\frac{1}{W e} t}\left(\frac{\partial u}{\partial z} \frac{\partial v}{\partial z}\right), \\
\frac{\partial \widetilde{S}^{x z}}{\partial t}=\frac{\partial u}{\partial z} \widetilde{S}^{z z}-\frac{1}{R e} e^{\frac{1}{W e} t} \frac{\partial}{\partial t}\left(\frac{\partial u}{\partial z}\right), \\
\frac{\partial z}{\partial z} \widetilde{S}^{z z}-\frac{1}{R e} e^{\frac{1}{W e} t} \frac{\partial}{\partial t}\left(\frac{\partial v}{\partial z}\right) .
\end{gathered}
$$

Da condição inicial $\mathbf{S}=\mathbf{0}$ e da equação (1.38), temos que no instante $t=0$, $\widetilde{\mathbf{S}}=\mathbf{S}=\mathbf{0}$. Logo, por (1.47), tem-se $\widetilde{S}^{z z}=0$, ou seja,

$$
S^{z z}=0
$$

Substituindo-se $S^{z z}=0$ nas equações (1.49) e (1.50), obtêm-se

$$
\frac{\partial \widetilde{S}^{x z}}{\partial t}=-\frac{1}{R e} e^{\frac{1}{W e} t} \frac{\partial}{\partial t}\left(\frac{\partial u}{\partial z}\right)
$$




$$
\frac{\partial \widetilde{S}^{y z}}{\partial t}=-\frac{1}{R e} e^{\frac{1}{W e} t} \frac{\partial}{\partial t}\left(\frac{\partial v}{\partial z}\right) .
$$

Integrando-se (1.52) e (1.53) no intervalo $[t, t+\delta t]$, vêm

$$
\begin{aligned}
& \widetilde{S}^{x z}(\mathbf{x}, t+\delta t)-\widetilde{S}^{x z}(\mathbf{x}, t)=-\frac{1}{R e} \int_{t}^{t+\delta t} e^{\frac{1}{W e} s} \frac{\partial}{\partial s}\left(\frac{\partial u}{\partial z}(\mathbf{x}, s)\right) d s \\
& \widetilde{S}^{y z}(\mathbf{x}, t+\delta t)-\widetilde{S}^{y z}(\mathbf{x}, t)=-\frac{1}{R e} \int_{t}^{t+\delta t} e^{\frac{1}{W e} s} \frac{\partial}{\partial s}\left(\frac{\partial v}{\partial z}(\mathbf{x}, s)\right) d s .
\end{aligned}
$$

Resolvendo as integrais nas equações (1.54) e (1.55) por partes têm-se

$$
\begin{array}{r}
\widetilde{S}^{x z}(\mathbf{x}, t+\delta t)-\widetilde{S}^{x z}(\mathbf{x}, t)=-\frac{1}{R e}\left(\frac{\partial u}{\partial z}(\mathbf{x}, t+\delta t) e^{\frac{1}{W e}(t+\delta t)}\right. \\
\left.-\frac{\partial u}{\partial z}(\mathbf{x}, t) e^{\frac{1}{W e} t}-\int_{t}^{t+\delta t} \frac{1}{W e} e^{\frac{1}{W e} s} \frac{\partial u}{\partial z}(\mathbf{x}, s) d s\right) \\
\widetilde{S}^{y z}(\mathbf{x}, t+\delta t)-\widetilde{S}^{y z}(\mathbf{x}, t)=-\frac{1}{R e}\left(\frac{\partial v}{\partial z}(\mathbf{x}, t+\delta t) e^{\frac{1}{W e}(t+\delta t)}\right. \\
\left.-\frac{\partial v}{\partial z}(\mathbf{x}, t) e^{\frac{1}{W e} t}-\int_{t}^{t+\delta t} \frac{1}{W e} e^{\frac{1}{W e} s} \frac{\partial v}{\partial z}(\mathbf{x}, s) d s\right) .
\end{array}
$$

Agora, aplicando-se o teorema do valor médio para integrais nas equações (1.56) e (1.57), obtêm-se

$$
\begin{array}{r}
\widetilde{S}^{x z}(\mathbf{x}, t+\delta t)-\widetilde{S}^{x z}(\mathbf{x}, t)=-\frac{1}{R e}\left(\frac{\partial u}{\partial z}(\mathbf{x}, t+\delta t) e^{\frac{1}{W e}(t+\delta t)}\right. \\
\left.-\frac{\partial u}{\partial z}(\mathbf{x}, t) e^{\frac{1}{W e} t}-\frac{\partial u}{\partial z}\left(\mathbf{x}, t^{*}\right) \int_{t}^{t+\delta t} \frac{1}{W e} e^{\frac{1}{W e} s} d s\right) \\
\widetilde{S}^{y z}(\mathbf{x}, t+\delta t)-\widetilde{S}^{y z}(\mathbf{x}, t)=-\frac{1}{R e}\left(\frac{\partial v}{\partial z}(\mathbf{x}, t+\delta t) e^{\frac{1}{W e}(t+\delta t)}\right. \\
\left.-\frac{\partial v}{\partial z}(\mathbf{x}, t) e^{\frac{1}{W e} t}-\frac{\partial v}{\partial z}\left(\mathbf{x}, t^{* *}\right) \int_{t}^{t+\delta t} \frac{1}{W e} e^{\frac{1}{W e} s} d s\right),
\end{array}
$$

com $t^{*}$ e $t^{* *}$ no intervalo $[t, t+\delta t]$. Uma vez que $t^{*}$ e $t^{* *}$ são desconhecidos, $\frac{\partial u}{\partial z}\left(\mathbf{x}, t^{*}\right)$ e $\frac{\partial v}{\partial z}\left(\mathbf{x}, t^{* *}\right)$ são aproximadas pela média aritmética entre seus respectivos valores nos instantes $t$ e $t+\delta t$. Assim, as equações (1.58) e (1.59) 
resultam, após o cálculo das integrais, em

$$
\begin{aligned}
\widetilde{S}^{x z}(\mathbf{x}, t+\delta t) & -\widetilde{S}^{x z}(\mathbf{x}, t)= \\
& -\frac{1}{R e}\left[\frac{\partial u}{\partial z}(\mathbf{x}, t+\delta t) e^{\frac{1}{W e}(t+\delta t)}-\frac{\partial u}{\partial z}(\mathbf{x}, t) e^{\frac{1}{W e} t}\right. \\
& \left.-\frac{1}{2}\left(\frac{\partial u}{\partial z}(\mathbf{x}, t)+\frac{\partial u}{\partial z}(\mathbf{x}, t+\delta t)\right)\left(e^{\frac{1}{W e}(t+\delta t)}-e^{\frac{1}{W e} t}\right)\right], \\
\widetilde{S}^{y z}(\mathbf{x}, t+\delta t) & -\widetilde{S}^{y z}(\mathbf{x}, t)= \\
& -\frac{1}{R e}\left[\frac{\partial v}{\partial z}(\mathbf{x}, t+\delta t) e^{\frac{1}{W e}(t+\delta t)}-\frac{\partial v}{\partial z}(\mathbf{x}, t) e^{\frac{1}{W e} t}\right. \\
& \left.-\frac{1}{2}\left(\frac{\partial v}{\partial z}(\mathbf{x}, t)+\frac{\partial v}{\partial z}(\mathbf{x}, t+\delta t)\right)\left(e^{\frac{1}{W e}(t+\delta t)}-e^{\frac{1}{W e} t}\right)\right] .
\end{aligned}
$$

Rearranjando-se os termos entre colchetes nas equações (1.60) e (1.61) e multiplicando-se o resultado por $e^{-\frac{1}{W e}(t+\delta t)}$, obtêm-se $S^{x z}$ e $S^{y z}$ :

$$
\begin{aligned}
S^{x z}(\mathbf{x}, t+\delta t)= & e^{-\frac{1}{W e} \delta t} S^{x z}(\mathbf{x}, t) \\
& +\frac{1}{2 R e}\left(e^{-\frac{1}{W e} \delta t}+1\right)\left(\frac{\partial u}{\partial z}(\mathbf{x}, t)-\frac{\partial u}{\partial z}(\mathbf{x}, t+\delta t)\right), \\
S^{y z}(\mathbf{x}, t+\delta t)= & e^{-\frac{1}{W e} \delta t} S^{y z}(\mathbf{x}, t) \\
& +\frac{1}{2 R e}\left(e^{-\frac{1}{W e} \delta t}+1\right)\left(\frac{\partial v}{\partial z}(\mathbf{x}, t)-\frac{\partial v}{\partial z}(\mathbf{x}, t+\delta t)\right) .
\end{aligned}
$$

Para obtermos $\widetilde{S}^{x x}$, integramos a equação (1.45) no intervalo $[t, t+\delta t]$, obtendo

$$
\begin{aligned}
\widetilde{S}^{x x}(\mathbf{x}, t+\delta t)-\widetilde{S}^{x x}(\mathbf{x}, t)= & 2 \int_{t}^{t+\delta t} \frac{\partial u}{\partial z}(\mathbf{x}, s) \widetilde{S}^{x z}(\mathbf{x}, s) d s \\
& +\frac{2}{R e} \int_{t}^{t+\delta t} e^{\frac{1}{W e} s}\left(\frac{\partial u}{\partial z}(\mathbf{x}, s)\right)^{2} d s
\end{aligned}
$$

Agora, aplicando-se, respectivamente, a fórmula do trapézio e o teorema do valor médio para integrais na primeira e segunda integrais da equação (1.64) 
obtemos

$$
\begin{aligned}
\widetilde{S}^{x x}(\mathbf{x}, t+\delta t)-\widetilde{S}^{x x}(\mathbf{x}, t) & =\delta t\left(\frac{\partial u}{\partial z}(\mathbf{x}, t+\delta t) \widetilde{S}^{x z}(\mathbf{x}, t+\delta t)\right. \\
\left.+\frac{\partial u}{\partial z}(\mathbf{x}, t) \widetilde{S}^{x z}(\mathbf{x}, t)\right) & +2 \frac{W e}{R e}\left(\frac{\partial u}{\partial z}\left(\mathbf{x}, t^{\dagger}\right)\right)^{2} e^{\frac{1}{W e} t}\left(e^{\frac{1}{W e} \delta t}-1\right)
\end{aligned}
$$

com $t^{\dagger}$ pertencente ao intervalo $[t, t+\delta t]$, de modo que a derivada é aproximada como na equação (1.60). Assim, da equação (1.65), o valor de $S^{x x}$ é obtido por

$$
\begin{aligned}
& S^{x x}(\mathbf{x}, t+\delta t)=e^{-\frac{1}{W e} \delta t} S^{x x}(\mathbf{x}, t) \\
& +\delta t\left(\frac{\partial u}{\partial z}(\mathbf{x}, t+\delta t) S^{x z}(\mathbf{x}, t+\delta t)+\frac{\partial u}{\partial z}(\mathbf{x}, t) e^{-\frac{1}{W e} \delta t} S^{x z}(\mathbf{x}, t)\right) \\
& \quad+\frac{1}{2} \frac{W e}{R e}\left(\frac{\partial u}{\partial z}(\mathbf{x}, t)+\frac{\partial u}{\partial z}(\mathbf{x}, t+\delta t)\right)^{2}\left(1-e^{-\frac{1}{W e} \delta t}\right)
\end{aligned}
$$

As equações (1.46) e (1.48) são tratadas de maneira análoga, de modo que $S^{y y}$ e $S^{x y}$ são obtidos, respectivamente, por

$$
\begin{aligned}
& S^{y y}(\mathbf{x}, t+\delta t)=e^{-\frac{1}{W e} \delta t} S^{y y}(\mathbf{x}, t) \\
&+ \delta t\left(\frac{\partial v}{\partial z}(\mathbf{x}, t+\delta t) S^{y z}(\mathbf{x}, t+\delta t)+\frac{\partial v}{\partial z}(\mathbf{x}, t) e^{-\frac{1}{W e} \delta t} S^{y z}(\mathbf{x}, t)\right) \\
&+ \frac{1}{2} \frac{W e}{R e}\left(\frac{\partial v}{\partial z}(\mathbf{x}, t)+\frac{\partial v}{\partial z}(\mathbf{x}, t+\delta t)\right)^{2}\left(1-e^{-\frac{1}{W e} \delta t}\right) \\
& S^{x y}(\mathbf{x}, t+\delta t)=e^{-\frac{1}{W e} \delta t} S^{x y}(\mathbf{x}, t) \\
&+\frac{\delta t}{2}\left(\frac{\partial u}{\partial z}(\mathbf{x}, t+\delta t) S^{y z}(\mathbf{x}, t+\delta t)+\frac{\partial u}{\partial z}(\mathbf{x}, t) e^{-\frac{1}{W e} \delta t} S^{y z}(\mathbf{x}, t)\right) \\
&+\frac{\delta t}{2}\left(\frac{\partial v}{\partial z}(\mathbf{x}, t+\delta t) S^{x z}(\mathbf{x}, t+\delta t)+\frac{\partial v}{\partial z}(\mathbf{x}, t) e^{-\frac{1}{W e} \delta t} S^{x z}(\mathbf{x}, t)\right) \\
&+\frac{1}{2} \frac{W e}{R e}\left(\frac{\partial u}{\partial z}(\mathbf{x}, t)+\frac{\partial u}{\partial z}(\mathbf{x}, t+\delta t)\right)\left(\frac{\partial v}{\partial z}(\mathbf{x}, t)+\frac{\partial v}{\partial z}(\mathbf{x}, t+\delta t)\right)\left(1-e^{-\frac{1}{W e} \delta t}\right) .
\end{aligned}
$$




\subsubsection{Contornos rígidos paralelos ao plano $x z$}

Nesses contornos temos $u=v=w=0$ (devido às condições de não-escorregamento e impermeabilidade) e $\frac{\partial u}{\partial x}=\frac{\partial v}{\partial x}=\frac{\partial w}{\partial x}=0, \frac{\partial u}{\partial z}=\frac{\partial v}{\partial z}=\frac{\partial w}{\partial z}=0$. Pela equação da continuidade (1.21), $\frac{\partial v}{\partial y}=0$. Dessa forma, $D^{x x}=D^{x z}=D^{y y}=$ $D^{z z}=0, D^{x y}=\frac{1}{2} \frac{\partial u}{\partial y}$ e $D^{y z}=\frac{1}{2} \frac{\partial w}{\partial y}$. Portanto as equações (1.39)-(1.44) se reduzem a

$$
\begin{gathered}
\frac{\partial \widetilde{S} x}{\partial t}=2 \frac{\partial u}{\partial y} \widetilde{S}^{x y}+\frac{2}{R e} e^{\frac{1}{W e} t}\left(\frac{\partial u}{\partial y}\right)^{2}, \\
\frac{\partial \widetilde{S}^{y y}}{\partial t}=0 \\
\frac{\partial \widetilde{S} z z}{\partial t}=2 \frac{\partial w}{\partial y} \widetilde{S}^{y z}+\frac{2}{R e} e^{\frac{1}{W e} t}\left(\frac{\partial w}{\partial y}\right)^{2}, \\
\frac{\partial \widetilde{S}^{x y}}{\partial t}=\frac{\partial u}{\partial y} \widetilde{S}^{y y}-\frac{1}{R e} e^{\frac{1}{W e} t} \frac{\partial}{\partial t}\left(\frac{\partial u}{\partial y}\right), \\
\frac{\partial u}{\partial y} \widetilde{S}^{y z}+\frac{\partial w}{\partial y} \widetilde{S}^{x y}+\frac{2}{R e} e^{\frac{1}{W e} t}\left(\frac{\partial u}{\partial y} \frac{\partial w}{\partial y}\right), \\
\frac{\partial t}{\partial t}=\frac{\partial w}{\partial y} \widetilde{S}^{y y}-\frac{1}{R e} e^{\frac{1}{W e} t} \frac{\partial}{\partial t}\left(\frac{\partial w}{\partial y}\right) .
\end{gathered}
$$

As componentes do tensor não-newtoniano $\mathbf{S}$ nesses contornos são obtidas por meio das equações (1.69)-(1.74) que são resolvidas de maneira análoga ao caso de contornos rígidos paralelos ao plano $x y$. Nesse caso, obtêm-se:

$$
S^{y y}=0
$$

$$
\begin{aligned}
S^{x y}(\mathbf{x}, t+\delta t)= & e^{-\frac{1}{W e} \delta t} S^{x y}(\mathbf{x}, t) \\
& +\frac{1}{2 R e}\left(e^{-\frac{1}{W e} \delta t}+1\right)\left(\frac{\partial u}{\partial y}(\mathbf{x}, t)-\frac{\partial u}{\partial y}(\mathbf{x}, t+\delta t)\right), \\
S^{y z}(\mathbf{x}, t+\delta t)= & e^{-\frac{1}{W e} \delta t} S^{y z}(\mathbf{x}, t) \\
& +\frac{1}{2 R e}\left(e^{-\frac{1}{W e} \delta t}+1\right)\left(\frac{\partial w}{\partial y}(\mathbf{x}, t)-\frac{\partial w}{\partial y}(\mathbf{x}, t+\delta t)\right),
\end{aligned}
$$




$$
\begin{aligned}
& S^{x x}(\mathbf{x}, t+\delta t)=e^{-\frac{1}{W e} \delta t} S^{x x}(\mathbf{x}, t) \\
&+ \delta t\left(\frac{\partial u}{\partial y}(\mathbf{x}, t+\delta t) S^{x y}(\mathbf{x}, t+\delta t)+\frac{\partial u}{\partial y}(\mathbf{x}, t) e^{-\frac{1}{W e} \delta t} S^{x y}(\mathbf{x}, t)\right) \\
&+ \frac{1}{2} \frac{W e}{R e}\left(\frac{\partial u}{\partial y}(\mathbf{x}, t)+\frac{\partial u}{\partial y}(\mathbf{x}, t+\delta t)\right)^{2}\left(1-e^{-\frac{1}{W e} \delta t}\right) \\
& S^{z z}(\mathbf{x}, t+\delta t)=e^{-\frac{1}{W e} \delta t} S^{z z}(\mathbf{x}, t) \\
&+\delta t\left(\frac{\partial w}{\partial y}(\mathbf{x}, t+\delta t) S^{y z}(\mathbf{x}, t+\delta t)+\frac{\partial w}{\partial y}(\mathbf{x}, t) e^{-\frac{1}{W e} \delta t} S^{y z}(\mathbf{x}, t)\right) \\
&+\frac{1}{2} \frac{W e}{R e}\left(\frac{\partial w}{\partial y}(\mathbf{x}, t)+\frac{\partial w}{\partial y}(\mathbf{x}, t+\delta t)\right)^{2}\left(1-e^{-\frac{1}{W e} \delta t}\right) \\
& S^{x z}(\mathbf{x}, t+\delta t)=e^{-\frac{1}{W e} \delta t} S^{x z}(\mathbf{x}, t) \\
&+\frac{\delta t}{2}\left(\frac{\partial u}{\partial y}(\mathbf{x}, t+\delta t) S^{y z}(\mathbf{x}, t+\delta t)+\frac{\partial u}{\partial y}(\mathbf{x}, t) e^{-\frac{1}{W e} \delta t} S^{y z}(\mathbf{x}, t)\right) \\
&+\frac{\delta t}{2}\left(\frac{\partial w}{\partial y}(\mathbf{x}, t+\delta t) S^{x y}(\mathbf{x}, t+\delta t)+\frac{\partial w}{\partial y}(\mathbf{x}, t) e^{-\frac{1}{W e} \delta t} S^{x y}(\mathbf{x}, t)\right) \\
&+\frac{1}{2} \frac{W e}{R e}\left(\frac{\partial u}{\partial y}(\mathbf{x}, t)+\frac{\partial u}{\partial y}(\mathbf{x}, t+\delta t)\right)\left(\frac{\partial w}{\partial y}(\mathbf{x}, t)+\frac{\partial w}{\partial y}(\mathbf{x}, t+\delta t)\right)\left(1-e^{-\frac{1}{W e} \delta t}\right)
\end{aligned}
$$

\subsubsection{Contornos rígidos paralelos ao plano $y z$}

Nesses contornos temos $u=v=w=0$, devido ao não-escorregamento e à impermeabilidade. Logo, $\frac{\partial u}{\partial y}=\frac{\partial v}{\partial y}=\frac{\partial w}{\partial y}=0, \frac{\partial u}{\partial z}=\frac{\partial v}{\partial z}=\frac{\partial w}{\partial z}=0$ e pela equação da continuidade (1.21), $\frac{\partial u}{\partial x}=0$. Dessa forma, $D^{x x}=D^{y y}=D^{y z}=$ $D^{z z}=0, D^{x y}=\frac{1}{2} \frac{\partial v}{\partial x}$ e $D^{x z}=\frac{1}{2} \frac{\partial w}{\partial x}$. Neste caso, as equações (1.39)-(1.44) se reduzem a

$$
\begin{gathered}
\frac{\partial \widetilde{S}^{x x}}{\partial t}=0 \\
\frac{\partial \widetilde{S}^{y y}}{\partial t}=2 \frac{\partial v}{\partial x} \widetilde{S}^{x y}+\frac{2}{R e} e^{\frac{1}{W e} t}\left(\frac{\partial v}{\partial x}\right)^{2} \\
\frac{\partial \widetilde{S}^{z z}}{\partial t}=2 \frac{\partial w}{\partial x} \widetilde{S}^{x z}+\frac{2}{R e} e^{\frac{1}{W e} t}\left(\frac{\partial w}{\partial x}\right)^{2} \\
\frac{\partial \widetilde{S}^{x y}}{\partial t}=\frac{\partial v}{\partial x} \widetilde{S}^{x x}-\frac{1}{R e} e^{\frac{1}{W e} t} \frac{\partial}{\partial t}\left(\frac{\partial v}{\partial x}\right)
\end{gathered}
$$




$$
\begin{gathered}
\frac{\partial \widetilde{S}^{x z}}{\partial t}=\frac{\partial w}{\partial x} \widetilde{S}^{x x}-\frac{1}{R e} e^{\frac{1}{W e} t} \frac{\partial}{\partial t}\left(\frac{\partial w}{\partial x}\right) \\
\frac{\partial \widetilde{S}^{y z}}{\partial t}=\frac{\partial v}{\partial x} \widetilde{S}^{x z}+\frac{\partial w}{\partial x} \widetilde{S}^{x y}+\frac{2}{R e} e^{\frac{1}{W e} t}\left(\frac{\partial v}{\partial x} \frac{\partial w}{\partial x}\right) .
\end{gathered}
$$

Como nos casos anteriores, obtêm-se, na ordem que segue, as componentes de $\mathbf{S}$ :

$$
\begin{aligned}
& S^{x x}=0, \\
& S^{x y}(\mathbf{x}, t+\delta t)=e^{-\frac{1}{W e} \delta t} S^{x y}(\mathbf{x}, t) \\
& +\frac{1}{2 R e}\left(e^{-\frac{1}{W e} \delta t}+1\right)\left(\frac{\partial v}{\partial x}(\mathbf{x}, t)-\frac{\partial v}{\partial x}(\mathbf{x}, t+\delta t)\right), \\
& S^{x z}(\mathbf{x}, t+\delta t)=e^{-\frac{1}{W e} \delta t} S^{x z}(\mathbf{x}, t) \\
& +\frac{1}{2 R e}\left(e^{-\frac{1}{W e} \delta t}+1\right)\left(\frac{\partial w}{\partial x}(\mathbf{x}, t)-\frac{\partial w}{\partial x}(\mathbf{x}, t+\delta t)\right), \\
& S^{y y}(\mathbf{x}, t+\delta t)=e^{-\frac{1}{W e} \delta t} S^{y y}(\mathbf{x}, t) \\
& +\delta t\left(\frac{\partial v}{\partial x}(\mathbf{x}, t+\delta t) S^{x y}(\mathbf{x}, t+\delta t)+\frac{\partial v}{\partial x}(\mathbf{x}, t) e^{-\frac{1}{W e} \delta t} S^{x y}(\mathbf{x}, t)\right) \\
& +\frac{1}{2} \frac{W e}{R e}\left(\frac{\partial v}{\partial x}(\mathbf{x}, t)+\frac{\partial v}{\partial x}(\mathbf{x}, t+\delta t)\right)^{2}\left(1-e^{-\frac{1}{W e} \delta t}\right), \\
& S^{z z}(\mathbf{x}, t+\delta t)=e^{-\frac{1}{W e} \delta t} S^{z z}(\mathbf{x}, t) \\
& +\delta t\left(\frac{\partial w}{\partial x}(\mathbf{x}, t+\delta t) S^{x z}(\mathbf{x}, t+\delta t)+\frac{\partial w}{\partial x}(\mathbf{x}, t) e^{-\frac{1}{W e} \delta t} S^{x z}(\mathbf{x}, t)\right) \\
& +\frac{1}{2} \frac{W e}{R e}\left(\frac{\partial w}{\partial x}(\mathbf{x}, t)+\frac{\partial w}{\partial x}(\mathbf{x}, t+\delta t)\right)^{2}\left(1-e^{-\frac{1}{W e} \delta t}\right), \\
& S^{y z}(\mathbf{x}, t+\delta t)=e^{-\frac{1}{W e} \delta t} S^{y z}(\mathbf{x}, t) \\
& +\frac{\delta t}{2}\left(\frac{\partial v}{\partial x}(\mathbf{x}, t+\delta t) S^{x z}(\mathbf{x}, t+\delta t)+\frac{\partial v}{\partial x}(\mathbf{x}, t) e^{-\frac{1}{W e} \delta t} S^{x z}(\mathbf{x}, t)\right) \\
& +\frac{\delta t}{2}\left(\frac{\partial w}{\partial x}(\mathbf{x}, t+\delta t) S^{x y}(\mathbf{x}, t+\delta t)+\frac{\partial w}{\partial x}(\mathbf{x}, t) e^{-\frac{1}{W e} \delta t} S^{x y}(\mathbf{x}, t)\right) \\
& +\frac{1}{2} \frac{W e}{R e}\left(\frac{\partial v}{\partial x}(\mathbf{x}, t)+\frac{\partial v}{\partial x}(\mathbf{x}, t+\delta t)\right)\left(\frac{\partial w}{\partial x}(\mathbf{x}, t)+\frac{\partial w}{\partial x}(\mathbf{x}, t+\delta t)\right)\left(1-e^{-\frac{1}{W e} \delta t}\right) .
\end{aligned}
$$




\subsection{Condições de contorno em superfícies livres}

Seja $\boldsymbol{\tau}$ o tensor das tensões totais, dado na forma adimensional por

$$
\boldsymbol{\tau}=-p \mathbf{I}+\mathbf{S}+\frac{2}{R e} \mathbf{D} .
$$

Neste trabalho, consideramos o escoamento de um fluido viscoso em uma atmosfera passiva e supomos que as tensões superficiais entre o fluido e a atmosfera são pequenas, podendo ser desprezadas. Dessa maneira, de acordo com Batchelor [39], as condições de contorno apropriadas em superfícies livres são dadas por

$$
\begin{aligned}
& \mathbf{n} \cdot(\boldsymbol{\tau} \cdot \mathbf{n})=0 \\
& \mathbf{m}_{1} \cdot(\boldsymbol{\tau} \cdot \mathbf{n})=0 \\
& \mathbf{m}_{2} \cdot(\boldsymbol{\tau} \cdot \mathbf{n})=0
\end{aligned}
$$

em que $\mathbf{n}$ é o vetor unitário normal e $\mathbf{m}_{1}$ e $\mathbf{m}_{2}$ são vetores unitários tangenciais à superficie livre. Fazendo $\mathbf{n}=\left(n_{x}, n_{y}, n_{z}\right)^{T}, \mathbf{m}_{1}=\left(m_{1 x}, m_{1 y}, m_{1 z}\right)^{T}$ e $\mathbf{m}_{2}=\left(m_{2 x}, m_{2 y}, m_{2 z}\right)^{T}$, as equações (1.94)-(1.96) são reescritas no espaço cartesiano tridimensional como

$$
\begin{aligned}
-p & +S^{x x} n_{x}^{2}+S^{y y} n_{y}^{2}+S^{z z} n_{z}^{2}+2\left(S^{x y} n_{x} n_{y}+S^{x z} n_{x} n_{z}+S^{y z} n_{y} n_{z}\right) \\
& +\frac{2}{R e}\left[\frac{\partial u}{\partial x} n_{x}^{2}+\frac{\partial v}{\partial y} n_{y}^{2}+\frac{\partial w}{\partial z} n_{z}^{2}+\left(\frac{\partial u}{\partial y}+\frac{\partial v}{\partial x}\right) n_{x} n_{y}\right. \\
& \left.+\left(\frac{\partial u}{\partial z}+\frac{\partial w}{\partial x}\right) n_{x} n_{z}+\left(\frac{\partial v}{\partial z}+\frac{\partial w}{\partial y}\right) n_{y} n_{z}\right]=0,
\end{aligned}
$$




$$
\begin{gathered}
S^{x x} n_{x} m_{1 x}+S^{y y} n_{y} m_{1 y}+S^{z z} n_{z} m_{1 z}+S^{x y}\left(n_{y} m_{1 x}+n_{x} m_{1 y}\right) \\
+S^{x z}\left(n_{z} m_{1 x}+n_{x} m_{1 z}\right)+S^{y z}\left(n_{z} m_{1 y}+n_{y} m_{1 z}\right)+\frac{1}{R e}\left[2 \frac{\partial u}{\partial x} n_{x} m_{1 x}\right. \\
+2 \frac{\partial v}{\partial y} n_{y} m_{1 y}+2 \frac{\partial w}{\partial z} n_{z} m_{1 z}+\left(\frac{\partial u}{\partial y}+\frac{\partial v}{\partial x}\right)\left(n_{y} m_{1 x}+n_{x} m_{1 y}\right) \\
\left.+\left(\frac{\partial u}{\partial z}+\frac{\partial w}{\partial x}\right)\left(n_{z} m_{1 x}+n_{x} m_{1 z}\right)+\left(\frac{\partial v}{\partial z}+\frac{\partial w}{\partial y}\right)\left(n_{z} m_{1 y}+n_{y} m_{1 z}\right)\right]=0 \\
S^{x x} n_{x} m_{2 x}+S^{y y} n_{y} m_{2 y}+S^{z z} n_{z} m_{2 z}+S^{x y}\left(n_{y} m_{2 x}+n_{x} m_{2 y}\right) \\
+S^{x z}\left(n_{z} m_{2 x}+n_{x} m_{2 z}\right)+S^{y z}\left(n_{z} m_{2 y}+n_{y} m_{2 z}\right)+\frac{1}{R e}\left[2 \frac{\partial u}{\partial x} n_{x} m_{2 x}\right. \\
+2 \frac{\partial v}{\partial y} n_{y} m_{2 y}+2 \frac{\partial w}{\partial z} n_{z} m_{2 z}+\left(\frac{\partial u}{\partial y}+\frac{\partial v}{\partial x}\right)\left(n_{y} m_{2 x}+n_{x} m_{2 y}\right) \\
\left.+\left(\frac{\partial u}{\partial z}+\frac{\partial w}{\partial x}\right)\left(n_{z} m_{2 x}+n_{x} m_{2 z}\right)+\left(\frac{\partial v}{\partial z}+\frac{\partial w}{\partial y}\right)\left(n_{z} m_{2 y}+n_{y} m_{2 z}\right)\right]=0 .
\end{gathered}
$$

As equações (1.97)-(1.99) constituem, desta forma, as condições de contorno a serem satisfeitas nas superfícies livres. 


\section{Capítulo 2}

\section{Método numérico}

Na forma vetorial, as equações adimensionais (1.21)-(1.30) são dadas por

$$
\begin{aligned}
& \nabla \cdot \mathbf{u}=0 \\
& \frac{\partial \mathbf{u}}{\partial t}=-\nabla \cdot(\mathbf{u u})-\nabla p+\frac{1}{R e} \nabla^{2} \mathbf{u}+\nabla \cdot \mathbf{S}+\frac{1}{F r^{2}} \mathbf{g}, \\
& \mathbf{S}+W e\left[\frac{\partial \mathbf{S}}{\partial t}+\nabla \cdot(\mathbf{u} \mathbf{S})-(\nabla \mathbf{u})^{T} \cdot \mathbf{S}-\mathbf{S} \cdot(\nabla \mathbf{u})\right]= \\
& -2 \frac{W e}{R e}\left[\frac{\partial \mathbf{D}}{\partial t}+\nabla \cdot(\mathbf{u D})-(\nabla \mathbf{u})^{T} \cdot \mathbf{D}-\mathbf{D} \cdot(\nabla \mathbf{u})\right] .
\end{aligned}
$$

O objetivo é resolver as equações (2.1)-(2.3) para as variáveis $\mathbf{u}, p$ e $\mathbf{S}$. Para isso, vamos adotar um algoritmo semelhante ao empregado por Tomé et al. [30] para escoamentos bidimensionais envolvendo o fluido Oldroyd-B. Esse algoritmo é baseado no método das projeções apresentado por Chorin $[36]$.

\subsection{Algoritmo computacional}

Suponhamos que $\mathbf{u}\left(\mathbf{x}, t_{n}\right)$ e $\mathbf{S}\left(\mathbf{x}, t_{n}\right)$ sejam conhecidos, juntamente com as condições de contorno. Então, $\mathbf{u}\left(\mathbf{x}, t_{n+1}\right), p\left(\mathbf{x}, t_{n+1}\right)$ e $\mathbf{S}\left(\mathbf{x}, t_{n+1}\right)$ podem ser obtidos como segue: 
Passo 1: Seja $\widetilde{p}\left(\mathbf{x}, t_{n}\right)$ um campo de pressão arbitrário que satisfaça a condição (1.97) na superfície livre.

Passo 2: Calcula-se o campo de velocidades intermediário, $\widetilde{\mathbf{u}}\left(\mathbf{x}, t_{n+1}\right)$, por meio da equação

$$
\frac{\partial \widetilde{\mathbf{u}}}{\partial t}=-\nabla \cdot(\mathbf{u u})-\nabla \widetilde{p}+\frac{1}{R e} \nabla^{2} \mathbf{u}+\nabla \cdot \mathbf{S}+\frac{1}{F r^{2}} \mathbf{g},
$$

com $\widetilde{\mathbf{u}}\left(\mathbf{x}, t_{n}\right)=\mathbf{u}\left(\mathbf{x}, t_{n}\right)$ inclusive para as condições de contorno.

Subtraindo-se (2.4) de (2.2), temos

$$
\frac{\partial(\mathbf{u}-\widetilde{\mathbf{u}})}{\partial t}=-\nabla(p-\widetilde{p})
$$

Aplicando-se o operador rotacional em (2.5), obtém-se

$$
\nabla \times\left(\frac{\partial(\mathbf{u}-\widetilde{\mathbf{u}})}{\partial t}\right)=-\nabla \times(\nabla(p-\widetilde{p}))=0
$$

Comutando-se os operadores em (2.6), vem

$$
\frac{\partial}{\partial t}[\nabla \times(\mathbf{u}-\widetilde{\mathbf{u}})]=0
$$

ou seja, $\nabla \times(\mathbf{u}-\widetilde{\mathbf{u}})=\mathbf{f}(\mathbf{x}), t \in\left[t_{n}, t_{n+1}\right]$. Porém, como $\widetilde{\mathbf{u}}\left(\mathbf{x}, t_{n}\right)=$ $\mathbf{u}\left(\mathbf{x}, t_{n}\right)$, conclui-se que $\mathbf{f}(\mathbf{x})=\mathbf{0}$ e portanto

$$
\nabla \times \widetilde{\mathbf{u}}\left(\mathbf{x}, t_{n+1}\right)=\nabla \times \mathbf{u}\left(\mathbf{x}, t_{n+1}\right)
$$

o que mostra que $\widetilde{\mathbf{u}}(\mathbf{x}, t)$ contém a vorticidade correta em $t=t_{n+1}$. Porém, seu cálculo através de (2.4) não garante que a equação da continuidade (2.1) seja satisfeita.

Seja $\psi\left(\mathbf{x}, t_{n+1}\right)$ uma função escalar que satisfaça a equação de Poisson

$$
\nabla^{2} \psi\left(\mathbf{x}, t_{n+1}\right)=\nabla \cdot \widetilde{\mathbf{u}}\left(\mathbf{x}, t_{n+1}\right)
$$


Define-se $\mathbf{u}\left(\mathbf{x}, t_{n+1}\right)$ por

$$
\mathbf{u}\left(\mathbf{x}, t_{n+1}\right)=\widetilde{\mathbf{u}}\left(\mathbf{x}, t_{n+1}\right)-\nabla \psi\left(\mathbf{x}, t_{n+1}\right)
$$

Logo, $\mathbf{u}\left(\mathbf{x}, t_{n+1}\right)$ contém a mesma vorticidade (gerada por $\widetilde{\mathbf{u}}(\mathbf{x}, t)$ ) e adicionalmente, conserva massa.

Passo 3: Calcula-se a equação de Poisson (2.9) com condições de contorno

- $\frac{\partial \psi}{\partial n}=0$ em entradas de fluido e contornos rígidos, em que $n$ denota sua direção normal; e

- $\psi=0$ na superfície livre.

Passo 4: Calcula-se a velocidade final $\mathbf{u}\left(\mathbf{x}, t_{n+1}\right)$ por meio de $(2.10)$.

Passo 5: Calcula-se a pressão final. Utilizando as equações (2.5) e (2.10) e após algumas considerações [3], mostra-se que

$$
p\left(\mathbf{x}, t_{n+1}\right)=\widetilde{p}\left(\mathbf{x}, t_{n}\right)+\frac{\psi\left(\mathbf{x}, t_{n+1}\right)}{\partial t} .
$$

Passo 6: Atualizam-se as componentes do tensor não-newtoniano S em entradas e saídas de fluido, conforme apresentado na Seção 1.3.

Passo 7: Atualizam-se as componentes do tensor não-newtoniano $\mathbf{S}$ em contornos rígidos, conforme apresentado na Seção 1.4.

Passo 8: Calculam-se as componentes do tensor não-newtoniano $\mathbf{S}$ através da equação (2.3). Isolando-se a derivada temporal, obtém-se a equação

$$
\begin{aligned}
\frac{\partial \mathbf{S}}{\partial t} & =-\nabla \cdot(\mathbf{u S})+(\nabla \mathbf{u})^{T} \cdot \mathbf{S}+\mathbf{S} \cdot(\nabla \mathbf{u}) \\
& -\frac{2}{R e}\left[\frac{\partial \mathbf{D}}{\partial t}+\nabla \cdot(\mathbf{u D})-(\nabla \mathbf{u})^{T} \cdot \mathbf{D}-\mathbf{D} \cdot(\nabla \mathbf{u})\right]-\frac{1}{W e} \mathbf{S}
\end{aligned}
$$


Passo 9: Atualizam-se as posições das partículas marcadoras que representam o fluido. Essas partículas permitem a visualização do escoamento e a orientação das superfícies livres, e suas posições são atualizadas integrando-se a equação

$$
\frac{\partial \mathbf{x}}{\partial t}=\mathbf{u}\left(\mathbf{x}, t_{n+1}\right)
$$

pelo método de Euler explícito. 


\section{Capítulo 3}

\section{Aproximação por diferenças finitas}

As equações (2.4), (2.9)-(2.13) são resolvidas pelo método de diferenças finitas em uma malha deslocada, com espaçamento $\delta x, \delta y$ e $\delta z$ nas direções $x$, $y$ e $z$, respectivamente.

As variáveis pressão, tensor taxa de deformação e tensor não-newtoniano são aproximadas no centro $(i, j, k)$ da célula enquanto que as componentes $u, v$ e $w$ da velocidade são aproximadas nas faces $(i+1 / 2, j, k),(i, j+1 / 2, k)$ e $(i, j, k+1 / 2)$, respectivamente, conforme representado na Figura 3.1.

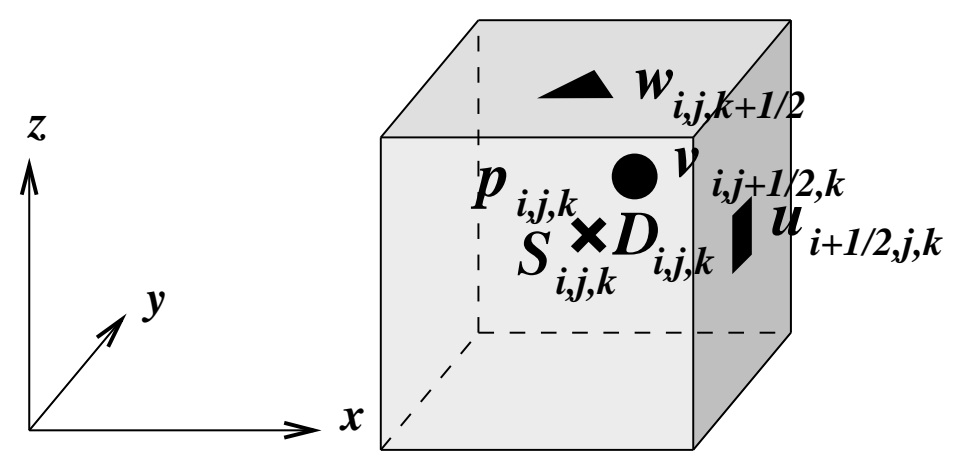

Figura 3.1: Célula computacional utilizada na discretização por diferenças finitas. 


\subsection{Classificação das células}

Nesse trabalho, pretendemos tratar de escoamentos onde o fluido está continuamente em movimento e portanto, torna-se necessário fazer uma classificação das células da malha para identificar a região do fluido. Também, é preciso identificar os vários tipos de fronteira (entrada de fluido, saída de fluido, contorno rígido e superfície livre) para a aplicação das condições de contorno apropriadas. Para tanto, adota-se a seguinte classificação de células:

- Células Vazias (E): São células que não contêm fluido;

- Células Cheias (F): São células cheias de fluido e que não possuem nenhuma face em contato com face de célula vazia;

- Células de Superfície (S): São células que contêm fluido e que possuem pelo menos uma face em contato com face de célula vazia;

- Células de Contorno (B): São células que definem um contorno rígido, de maneira que as condições de contorno possam ser impostas;

- Células Inflow (I): São células que definem as entradas de fluido;

- Células Outflow (O): São células que definem as saídas de fluido.

A Figura 3.2 ilustra a estrutura de células acima descrita.

\subsection{Aproximação da equação de conservação de quantidade de movimento}

Em coordenadas cartesianas tridimensionais, a equação (2.4) é dada por

$$
\begin{aligned}
\frac{\partial \widetilde{u}}{\partial t}= & -\frac{\partial\left(u^{2}\right)}{\partial x}-\frac{\partial(v u)}{\partial y}-\frac{\partial(w u)}{\partial z}-\frac{\partial \widetilde{p}}{\partial x} \\
& +\frac{1}{R e}\left(\frac{\partial^{2} u}{\partial x^{2}}+\frac{\partial^{2} u}{\partial y^{2}}+\frac{\partial^{2} u}{\partial z^{2}}\right)+\frac{\partial S^{x x}}{\partial x}+\frac{\partial S^{x y}}{\partial y}+\frac{\partial S^{x z}}{\partial z}+\frac{1}{F r^{2}} g_{x}
\end{aligned}
$$




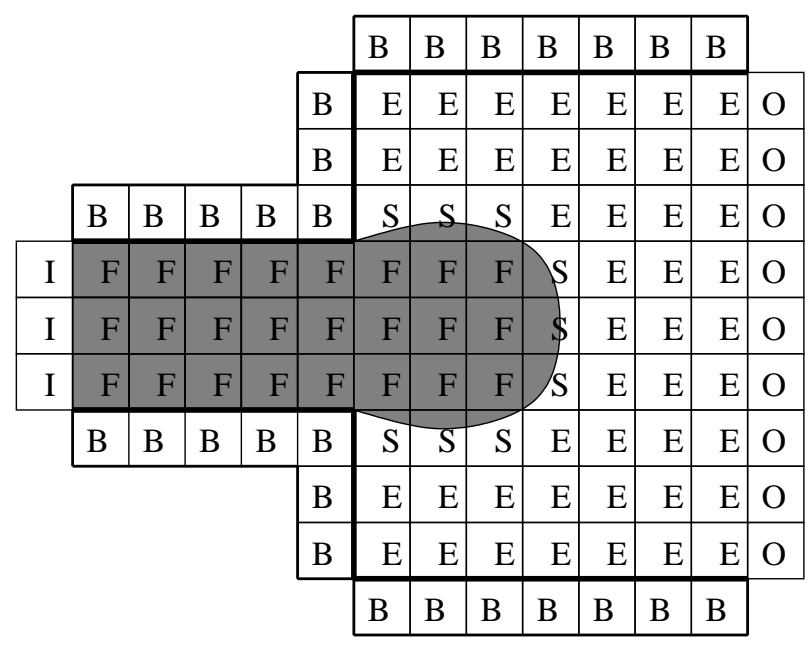

Figura 3.2: Tipos de célula no domínio computacional.

$$
\begin{aligned}
\frac{\partial \widetilde{v}}{\partial t}= & -\frac{\partial(u v)}{\partial x}-\frac{\partial\left(v^{2}\right)}{\partial y}-\frac{\partial(w v)}{\partial z}-\frac{\partial \widetilde{p}}{\partial y} \\
& +\frac{1}{R e}\left(\frac{\partial^{2} v}{\partial x^{2}}+\frac{\partial^{2} v}{\partial y^{2}}+\frac{\partial^{2} v}{\partial z^{2}}\right)+\frac{\partial S^{x y}}{\partial x}+\frac{\partial S^{y y}}{\partial y}+\frac{\partial S^{y z}}{\partial z}+\frac{1}{F r^{2}} g_{y} \\
\frac{\partial \widetilde{w}}{\partial t}= & -\frac{\partial(u w)}{\partial x}-\frac{\partial(v w)}{\partial y}-\frac{\partial\left(w^{2}\right)}{\partial z}-\frac{\partial \widetilde{p}}{\partial z} \\
& +\frac{1}{R e}\left(\frac{\partial^{2} w}{\partial x^{2}}+\frac{\partial^{2} w}{\partial y^{2}}+\frac{\partial^{2} w}{\partial z^{2}}\right)+\frac{\partial S^{x z}}{\partial x}+\frac{\partial S^{y z}}{\partial y}+\frac{\partial S^{z z}}{\partial z}+\frac{1}{F r^{2}} g_{z}
\end{aligned}
$$

As equações (3.1)-(3.3) são discretizadas nos pontos $(i+1 / 2, j, k),(i, j+$ $1 / 2, k)$ e $(i, j, k+1 / 2)$, respectivamente, sendo calculadas em todas as faces de células cheias $(\mathrm{F})$ ou de superfície $(\mathrm{S})$ que estejam em contato com outras faces de células F ou S.

As derivadas espaciais de primeira ordem envolvendo o tensor $\mathbf{S}$ e a pressão $\widetilde{p}$ são discretizadas por diferenças centrais. Os termos difusivos envolvendo a velocidade $\mathbf{u}$ são aproximados por diferenças centrais de segunda ordem, enquanto que os convectivos são tratados por meio do método upwind de alta ordem CUBISTA [38] (ver seção 3.7). Dessa maneira, a equação (2.4) 
é aproximada por um sistema de equações da forma

$$
\widetilde{\mathbf{u}}^{n+1}=\mathbf{u}^{n}+\delta t \mathbf{F}^{n}(\mathbf{u})
$$

onde $\mathbf{F}^{n}(\mathbf{u})=\left(F_{1}, F_{2}, F_{3}\right)^{T}$. A primeira componente, $F_{1}$ é dada por:

$$
\begin{aligned}
F_{1}= & -\operatorname{conv}\left(u^{2}\right)_{i+1 / 2, j, k}-\operatorname{conv}(v u)_{i+1 / 2, j, k}-\operatorname{conv}(w u)_{i+1 / 2, j, k} \\
& -\frac{\widetilde{p}_{i+1, j, k}-\widetilde{p}_{i, j, k}}{\delta x}+\frac{1}{R e}\left(\frac{u_{i-1 / 2, j, k}-2 u_{i+1 / 2, j, k}+u_{i+3 / 2, j, k}}{\delta x^{2}}\right. \\
& +\frac{u_{i+1 / 2, j-1, k}-2 u_{i+1 / 2, j, k}+u_{i+1 / 2, j+1, k}}{\delta y^{2}} \\
& \left.+\frac{u_{i+1 / 2, j, k-1}-2 u_{i+1 / 2, j, k}+u_{i+1 / 2, j, k+1}}{\delta z^{2}}\right)+\left(\frac{\partial S^{x x}}{\partial x}\right)_{i+1 / 2, j, k} \\
& +\left(\frac{\partial S^{x y}}{\partial y}\right)_{i+1 / 2, j, k}+\left(\frac{\partial S^{x z}}{\partial z}\right)_{i+1 / 2, j, k}+\frac{1}{F r^{2}} g_{x},
\end{aligned}
$$

onde

$$
\begin{aligned}
& \left(\frac{\partial S^{x x}}{\partial x}\right)_{i+1 / 2, j, k}=\frac{S_{i+1, j, k}^{x x}-S_{i, j, k}^{x x}}{\delta x} \\
& \left(\frac{\partial S^{x y}}{\partial y}\right)_{i+1 / 2, j, k}=\frac{S_{i+1 / 2, j+1 / 2, k}^{x y}-S_{i+1 / 2, j-1 / 2, k}^{x y}}{\delta y} \\
& \left(\frac{\partial S^{x z}}{\partial z}\right)_{i+1 / 2, j, k}=\frac{S_{i+1 / 2, j, k+1 / 2}^{x z}-S_{i+1 / 2, j, k-1 / 2}^{x z}}{\delta z} .
\end{aligned}
$$

O tratamento dos termos convectivos (representados por expressões do tipo $\left.\operatorname{conv}\left(u^{2}\right)_{i+1 / 2, j, k}\right)$ será discutido em detalhes na seção 3.7.

Note que o cálculo de $F_{1}$ portanto necessita de valores do tensor $\mathbf{S}$ que se encontram em pontos em que este não está definido pela discretização (equações (3.7) e (3.8)). Nesse caso, os valores de $S_{i+1 / 2, j+1 / 2, k}^{x y}, S_{i+1 / 2, j-1 / 2, k}^{x y}$, $S_{i+1 / 2, j, k+1 / 2}^{x z}$ e $S_{i+1 / 2, j, k-1 / 2}^{x z}$ são obtidos por medias aritméticas envolvendo os pontos vizinhos, por exemplo,

$$
S_{i+1 / 2, j+1 / 2, k}^{x y}=\frac{S_{i, j, k}^{x y}+S_{i+1, j, k}^{x y}+S_{i, j+1, k}^{x y}+S_{i+1, j+1, k}^{x y}}{4} .
$$


Por outro lado, se por exemplo no caso da equação (3.7) uma das células $(i, j, k)$ ou $(i+1, j, k)$ estiver em contato com alguma célula $\mathrm{B}, \mathrm{I}, \mathrm{O}$ ou E na direção $y$, então a derivada em questão será aproximada por uma diferença de primeira ordem. Se a célula $(i, j+1, k)$ for B, então

$$
\left(\frac{\partial S^{x y}}{\partial y}\right)_{i+1 / 2, j, k}=\frac{S_{i, j, k}^{x y}-S_{i, j-1, k}^{x y}}{\delta y},
$$

onde

$$
S_{i, j, k}^{x y}=\frac{S_{i, j, k}^{x y}+S_{i+1, j, k}^{x y}}{2}, \quad S_{i, j-1, k}^{x y}=\frac{S_{i, j-1, k}^{x y}+S_{i+1, j-1, k}^{x y}}{2}
$$

As outras componentes da equação (3.4) são aproximadas de maneira análoga.

\subsection{Aproximação da equação de Poisson}

Em coordenadas cartesianas tridimensionais, a equação (2.9) é dada por

$$
\frac{\partial^{2} \psi}{\partial x^{2}}+\frac{\partial^{2} \psi}{\partial y^{2}}+\frac{\partial^{2} \psi}{\partial z^{2}}=\frac{\partial \widetilde{u}}{\partial x}+\frac{\partial \widetilde{v}}{\partial y}+\frac{\partial \widetilde{w}}{\partial z}
$$

Aproximando-se as derivadas por diferenças finitas de segunda ordem, obtém-se

$$
\begin{aligned}
& \frac{\psi_{i-1, j, k}-2 \psi_{i, j, k}+\psi_{i+1, j, k}}{\delta x^{2}}+\frac{\psi_{i, j-1, k}-2 \psi_{i, j, k}+\psi_{i, j+1, k}}{\delta y^{2}} \\
& +\frac{\psi_{i, j, k-1}-2 \psi_{i, j, k}+\psi_{i, j, k+1}}{\delta z^{2}}=\widetilde{D}_{i, j, k},
\end{aligned}
$$

onde

$$
\begin{aligned}
\widetilde{D}_{i, j, k}= & \frac{\widetilde{u}_{i+1 / 2, j, k}-\widetilde{u}_{i-1 / 2, j, k}}{\delta x}+\frac{\widetilde{v}_{i, j+1 / 2, k}-\widetilde{v}_{i, j-1 / 2, k}}{\delta y} \\
& +\frac{\widetilde{w}_{i, j, k+1 / 2}-\widetilde{w}_{i, j, k-1 / 2}}{\delta z}
\end{aligned}
$$


A equação de Poisson (3.11) é aplicada em todas as células cheias do domínio computacional, gerando um sistema linear da forma $\mathbf{A x}=\mathbf{b}$ de ordem $n$, onde $n$ é o número de células cheias (F) da malha. Pode-se mostrar que A é uma matriz simétrica, definida positiva e esparsa, e b é o vetor divergente obtido a partir de $\widetilde{D}_{i, j, k}$. O método dos gradientes conjugados é utilizado para obter a solução desse sistema.

\subsection{Aproximação da equação da velocidade final}

A equação (2.10) em coordenadas cartesianas tridimensionais fornece as equações:

$$
\begin{aligned}
u\left(\mathbf{x}, t_{n+1}\right) & =\widetilde{u}\left(\mathbf{x}, t_{n+1}\right)-\frac{\partial \psi\left(\mathbf{x}, t_{n+1}\right)}{\partial x} \\
v\left(\mathbf{x}, t_{n+1}\right) & =\widetilde{v}\left(\mathbf{x}, t_{n+1}\right)-\frac{\partial \psi\left(\mathbf{x}, t_{n+1}\right)}{\partial y}, \\
w\left(\mathbf{x}, t_{n+1}\right) & =\widetilde{w}\left(\mathbf{x}, t_{n+1}\right)-\frac{\partial \psi\left(\mathbf{x}, t_{n+1}\right)}{\partial z} .
\end{aligned}
$$

Aplicando as equações (3.13)-(3.15) nos pontos $(i+1 / 2, j, k),(i, j+1 / 2, k)$ e $(i, j, k+1 / 2)$, respectivamente, obtêm-se as equações de diferenças finitas

$$
\begin{aligned}
& u_{i+1 / 2, j, k}^{n+1}=\widetilde{u}_{i+1 / 2, j, k}^{n+1}-\frac{\psi_{i+1, j, k}^{n+1}-\psi_{i, j, k}^{n+1}}{\delta x}, \\
& v_{i, j+1 / 2, k}^{n+1}=\widetilde{v}_{i, j+1 / 2, k}^{n+1}-\frac{\psi_{i, j+1, k}^{n+1}-\psi_{i, j, k}^{n+1}}{\delta y} \\
& w_{i, j, k+1 / 2}^{n+1}=\widetilde{w}_{i, j, k+1 / 2}^{n+1}-\frac{\psi_{i, j, k+1}^{n+1}-\psi_{i, j, k}^{n+1}}{\delta z} .
\end{aligned}
$$

\subsection{Aproximação da equação da pressão}

A equação (2.11) aplicada no ponto $(i, j, k)$ é dada por

$$
p_{i, j, k}^{n+1}=\widetilde{p}_{i, j, k}+\frac{\psi_{i, j, k}^{n+1}}{\delta t} .
$$




\subsection{Aproximação da equação constitutiva}

Na equação constitutiva (2.12), os termos lineares são aproximados por diferenças de segunda ordem, a derivada temporal pelo método de Euler explícito, e os termos convectivos são aproximados pelo esquema CUBISTA [38] (ver seção 3.7). Dessa forma, a equação (2.12) pode ser escrita como:

$$
\mathbf{S}^{n+1}=\mathbf{S}^{n}+\delta t \mathbf{H}(\mathbf{S})
$$

onde $\mathbf{H}(\mathbf{S})=\left(H_{1}, H_{2}, H_{3}, H_{4}, H_{5}, H_{6}\right)^{T}$.

Por exemplo, a componente $H_{1}$ é dada por

$$
\begin{aligned}
H_{1}= & -\operatorname{conv}\left(u S^{x x}\right)_{i, j, k}-\operatorname{conv}\left(v S^{x x}\right)_{i, j, k}-\operatorname{conv}\left(w S^{x x}\right)_{i, j, k} \\
& +2\left(\frac{\partial u}{\partial x}\right)_{i, j, k} S_{i, j, k}^{x x}+2\left(\frac{\partial u}{\partial y}\right)_{i, j, k} S_{i, j, k}^{x y}+2\left(\frac{\partial u}{\partial z}\right)_{i, j, k} S_{i, j, k}^{x z} \\
& -\frac{2}{R e}\left[\operatorname{conv}\left(u D^{x x}\right)_{i, j, k}+\operatorname{conv}\left(v D^{x x}\right)_{i, j, k}+\operatorname{conv}\left(w D^{x x}\right)_{i, j, k}\right. \\
& \left.-2\left(\frac{\partial u}{\partial x}\right)_{i, j, k} D_{i, j, k}^{x x}-2\left(\frac{\partial u}{\partial y}\right)_{i, j, k} D_{i, j, k}^{x y}-2\left(\frac{\partial u}{\partial z}\right)_{i, j, k} D_{i, j, k}^{x z}\right] \\
& -\frac{1}{W e} S_{i, j, k}^{x x}
\end{aligned}
$$

onde

$$
\begin{aligned}
& \left(\frac{\partial u}{\partial x}\right)_{i, j, k}=\frac{u_{i+1 / 2, j, k}-u_{i-1 / 2, j, k}}{\delta x}, \\
& \left(\frac{\partial u}{\partial y}\right)_{i, j, k}=\frac{u_{i, j+1 / 2, k}-u_{i, j-1 / 2, k}}{\delta y}, \\
& \left(\frac{\partial u}{\partial z}\right)_{i, j, k}=\frac{u_{i, j, k+1 / 2}-u_{i, j, k-1 / 2}}{\delta z} .
\end{aligned}
$$

Por simplicidade, a taxa de variação temporal do tensor taxa de deformação é suposta pequena e portanto não foi considerada na discretização. Observamos que o cálculo das derivadas cruzadas da equação (3.21) - por exemplo $\frac{\partial u}{\partial y}$ - requer valores de componentes da velocidade em pontos em que estas não estão definidas pela discretização. Esses valores são obtidos 
fazendo-se médias aritméticas envolvendo os pontos vizinhos. Por exemplo, se a célula $(i, j, k)$ não tiver como vizinhas células vazias na direção y então o valor de $u_{i, j+1 / 2, k}$ na equação (3.23) é calculado por

$$
u_{i, j+1 / 2, k}=\frac{u_{i-1 / 2, j, k}+u_{i+1 / 2, j, k}+u_{i-1 / 2, j+1, k}+u_{i+1 / 2, j+1, k}}{4} .
$$

O valor de $u_{i, j-1 / 2, k}$ é obtido de forma análoga.

Caso contrário, a derivada em questão é aproximada utilizando diferenças de primeira ordem. Por exemplo, se a célula $(i, j+1, k)$ for vazia então $\frac{\partial u}{\partial y}$ é aproximada por

$$
\left(\frac{\partial u}{\partial y}\right)_{i, j, k}=\frac{u_{i, j, k}-u_{i, j-1, k}}{\delta y}
$$

onde

$$
u_{i, j, k}=\frac{u_{i-1 / 2, j, k}+u_{i+1 / 2, j, k}}{2}, \quad u_{i, j-1, k}=\frac{u_{i-1 / 2, j-1, k}+u_{i+1 / 2, j-1, k}}{2} .
$$

\subsection{Discretização dos termos convectivos}

Os termos convectivos são responsáves por muitos fenômenos presentes nos escoamentos, e o tratamento adequado desses termos pode evitar o aparecimento de instabilidades numéricas nas simulações. Nesse trabalho vamos utilizar o esquema convectivo limitado de alta ordem denominado CUBISTA - a Convergent and Universally Bounded Interpolation Scheme for the Treatment of Advection, desenvolvido por Alves et al. [38]. Em seu trabalho, os autores empregaram o método CUBISTA para simular o escoamento de um fluido de Maxwell em uma contração planar, obtendo uma boa precisão nos resultados numéricos. A seguir, apresentamos uma idéia geral do método CUBISTA; detalhes das aplicações e implementação computacional podem ser encontrados em [40].

Considere a figura 3.3 para aproximar a derivada parcial $\frac{\partial(V \phi)}{\partial s}$ da variável genérica $\phi$ no ponto $P_{0}$. A variável $s$ representa uma direção dos eixos coordenados, sendo $\phi_{A}, \phi_{B}$ os valores da variável genérica e $V_{A}, V_{B}$ os valores da velocidade de convecção nos pontos $P_{A}$ e $P_{B}$ respectivamente. A derivada 
$\frac{\partial(V \phi)}{\partial s}$ é aproximada pela expressão:

$$
\left.\frac{\partial(V \phi)}{\partial s}\right|_{P_{0}}=\frac{V_{B} \phi_{B}-V_{A} \phi_{A}}{\Delta s} .
$$

Os valores de $\phi_{A}$ e $\phi_{B}$ são obtidos em termos dos valores vizinhos, $\phi_{-2}$, $\phi_{-1}, \phi_{0}, \phi_{1}, \phi_{2}$ e das velocidades convectivas $V_{A}$ e $V_{B}$ pelo método CUBISTA apresentado a seguir:

\section{CUBISTA:}

$$
\begin{gathered}
\text { Se } V_{B} \geq 0, \quad \phi_{B}= \begin{cases}\phi_{0}, & \hat{\phi}_{0} \notin[0,1] \\
\frac{7}{4} \phi_{0}-\frac{3}{4} \phi_{-1}, & \hat{\phi}_{0} \in[0,3 / 8) \\
\frac{3}{8} \phi_{1}+\frac{3}{4} \phi_{0}-\frac{1}{8} \phi_{-1}, & \hat{\phi}_{0} \in[3 / 8,3 / 4] \\
\frac{3}{4} \phi_{1}+\frac{1}{4} \phi_{0}, & \hat{\phi}_{0} \in(3 / 4,1]\end{cases} \\
\text { se } V_{B}<0, \quad \phi_{B}= \begin{cases}\phi_{1}, & \hat{\phi}_{1} \notin[0,1] \\
\frac{7}{4} \phi_{1}-\frac{3}{4} \phi_{2}, & \hat{\phi}_{1} \in[0,3 / 8) \\
\frac{3}{8} \phi_{0}+\frac{3}{4} \phi_{1}-\frac{1}{8} \phi_{2}, & \hat{\phi}_{1} \in[3 / 8,3 / 4] \\
\frac{3}{4} \phi_{0}+\frac{1}{4} \phi_{1}, & \hat{\phi}_{1} \in(3 / 4,1]\end{cases} \\
\text { se } V_{A} \geq 0, \quad \hat{\phi}_{A}= \begin{cases}\phi_{-1}, & \hat{\phi}_{-1} \in[0,1] \\
\frac{7}{4} \phi_{-1}-\frac{3}{4} \phi_{-2}, & \hat{\phi}_{-1} \in[3 / 8,3 / 4] \\
\frac{3}{8} \phi_{0}+\frac{3}{4} \phi_{-1}-\frac{1}{8} \phi_{-2}, & \hat{\phi}_{-1} \in(3 / 4,1] \\
\frac{3}{4} \phi_{0}+\frac{1}{4} \phi_{-1}, & \hat{\phi}_{0} \notin[0,1] \\
\text { se } V_{A}<0, & \phi_{A}= \begin{cases}\phi_{0}, & \hat{\phi}_{0} \in[0,3 / 8) \\
\frac{7}{4} \phi_{0}-\frac{3}{4} \phi_{1}, & \hat{\phi}_{0} \in[3 / 8,3 / 4] \\
\frac{3}{8} \phi_{-1}+\frac{3}{4} \phi_{0}-\frac{1}{8} \phi_{1}, \\
\frac{3}{4} \phi_{-1}+\frac{1}{4} \phi_{0},\end{cases} \end{cases}
\end{gathered}
$$

onde $\hat{\phi}_{i}, i=-1,0,1$, é definido em função das velocidades upstream $\left(\phi_{U}\right)$, remote upstream $\left(\phi_{R}\right)$ e downstream $\left(\phi_{D}\right)$ nos pontos $P_{A}$ e $P_{B}$, isto é, de 
acordo com a velocidade convectiva, sendo definido por

$$
\hat{\phi}_{i}=\frac{\phi_{U}-\phi_{R}}{\phi_{D}-\phi_{R}}
$$

Por exemplo, considerando-se o ponto $P_{A}$ como mostrado na figura 3.3, entre os nós -1 e 0, e assumindo-se que a velocidade em tal ponto é positiva $\left(V_{A}>0\right)$, os nós $D, U$ e $R$ correspondem aos nós $0,-1$ e -2 , respectivamente.

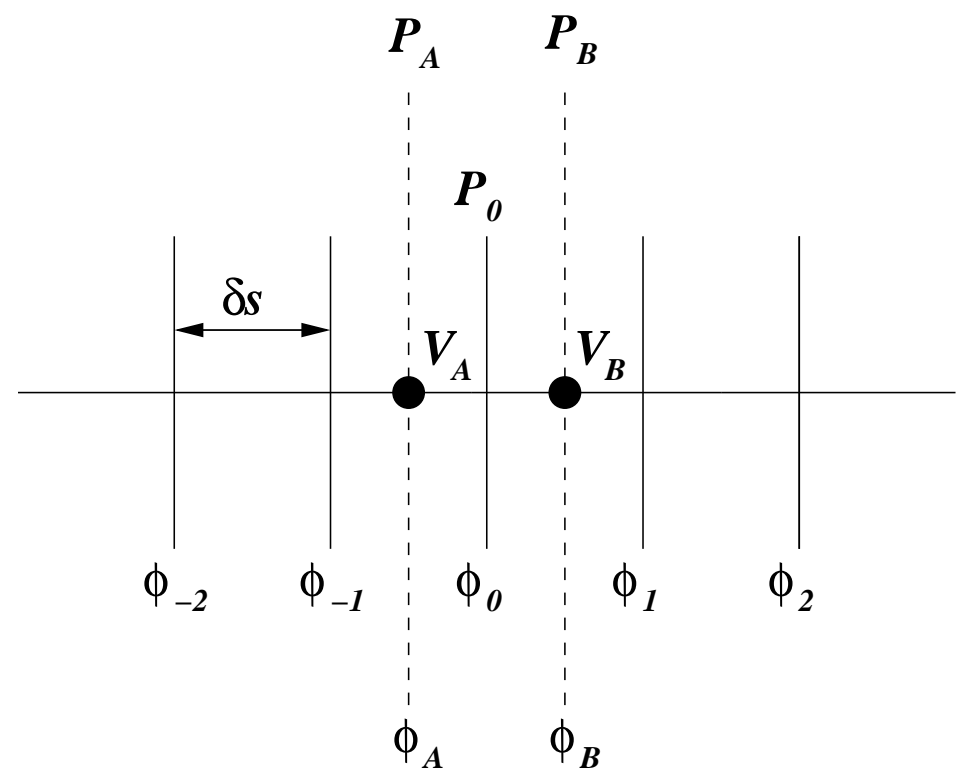

Figura 3.3: Estêncil utilizado para obter $\phi_{A}$ e $\phi_{B}$ através de CUBISTA.

Retornando ao caso de estudo, temos que, por exemplo, na equação (3.1), o termo convectivo $\frac{\partial(v u)}{\partial y}$ é aproximado em $(i+1 / 2, j, k)$ (denotado por $\operatorname{conv}(v u)_{i+1 / 2, j, k}$, ver equação (3.5)) da seguinte forma:

$$
\begin{aligned}
\left.\frac{\partial(v u)}{\partial y}\right|_{i+1 / 2, j, k} & \approx \operatorname{conv}(v u)_{i+1 / 2, j, k} \\
& =\frac{\bar{v}_{i+1 / 2, j+1 / 2, k} u_{i+1 / 2, j+1 / 2, k}-\bar{v}_{i+1 / 2, j-1 / 2, k} u_{i+1 / 2, j-1 / 2, k}}{\delta y},
\end{aligned}
$$

onde $\bar{v}$ é a velocidade convectiva calculada pela média aritmética dos valores 
vizinhos:

$$
\bar{v}_{i+1 / 2, j+1 / 2, k}=\frac{v_{i, j+1 / 2, k}+v_{i+1, j+1 / 2, k}}{2}, \quad \bar{v}_{i+1 / 2, j-1 / 2, k}=\frac{v_{i, j-1 / 2, k}+v_{i+1, j-1 / 2, k}}{2} .
$$

As velocidades transportadas $u$ são calculadas por meio do esquema CUBISTA como segue:

- $u_{i+1 / 2, j+1 / 2, k}$ :

- se $\bar{v}_{i+1 / 2, j+1 / 2, k} \geq 0$, então $\hat{u}_{i+1 / 2, j, k}=\frac{u_{i+1 / 2, j, k}-u_{i+1 / 2, j-1, k}}{u_{i+1 / 2, j+1, k}-u_{i+1 / 2, j-1, k}}$,

$$
u_{i+1 / 2, j+1 / 2, k}=\left\{\begin{array}{ll}
u_{i+1 / 2, j, k}, & \hat{u}_{i+1 / 2, j, k} \notin[0,1] \\
\frac{7}{4} u_{i+1 / 2, j, k}-\frac{3}{4} u_{i+1 / 2, j-1, k}, & \hat{u}_{i+1 / 2, j, k} \in[0,3 / 8) \\
\frac{3}{8} u_{i+1 / 2, j+1, k}+\frac{3}{4} u_{i+1 / 2, j, k}-\frac{1}{8} u_{i+1 / 2, j-1, k}, & \hat{u}_{i+1 / 2, j, k} \in[3 / 8,3 / 4] \\
\frac{3}{4} u_{i+1 / 2, j+1, k}+\frac{1}{4} u_{i+1 / 2, j, k}, & \hat{u}_{i+1 / 2, j, k} \in(3 / 4,1]
\end{array} ;\right.
$$

- se $\bar{v}_{i+1 / 2, j+1 / 2, k}<0$, então $\hat{u}_{i+1 / 2, j+1, k}=\frac{u_{i+1 / 2, j+1, k}-u_{i+1 / 2, j+2, k}}{u_{i+1 / 2, j, k}-u_{i+1 / 2, j+2, k}}$,

$$
u_{i+1 / 2, j+1 / 2, k}=\left\{\begin{array}{ll}
u_{i+1 / 2, j+1, k}, & \hat{u}_{i+1 / 2, j+1, k} \notin[0,1] \\
\frac{7}{4} u_{i+1 / 2, j+1, k}-\frac{3}{4} u_{i+1 / 2, j+2, k}, & \hat{u}_{i+1 / 2, j+1, k} \in[0,3 / 8) \\
\frac{3}{8} u_{i+1 / 2, j, k}+\frac{3}{4} u_{i+1 / 2, j+1, k}-\frac{1}{8} u_{i+1 / 2, j+2, k}, & \hat{u}_{i+1 / 2, j+1, k} \in[3 / 8,3 / 4] \\
\frac{3}{4} u_{i+1 / 2, j, k}+\frac{1}{4} u_{i+1 / 2, j+1, k}, & \hat{u}_{i+1 / 2, j+1, k} \in(3 / 4,1]
\end{array} .\right.
$$

- $u_{i+1 / 2, j-1 / 2, k}$ :

- se $\bar{v}_{i+1 / 2, j-1 / 2, k} \geq 0$, então $\hat{u}_{i+1 / 2, j-1, k}=\frac{u_{i+1 / 2, j-1, k}-u_{i+1 / 2, j-2, k}}{u_{i+1 / 2, j, k}-u_{i+1 / 2, j-2, k}}$,

$$
u_{i+1 / 2, j-1 / 2, k}=\left\{\begin{array}{ll}
u_{i+1 / 2, j-1, k}, & \hat{u}_{i+1 / 2, j-1, k} \notin[0,1] \\
\frac{7}{4} u_{i+1 / 2, j-1, k}-\frac{3}{4} u_{i+1 / 2, j-2, k}, & \hat{u}_{i+1 / 2, j-1, k} \in[0,3 / 8) \\
\frac{3}{8} u_{i+1 / 2, j, k}+\frac{3}{4} u_{i+1 / 2, j-1, k}-\frac{1}{8} u_{i+1 / 2, j-2, k}, & \hat{u}_{i+1 / 2, j-1, k} \in[3 / 8,3 / 4] \\
\frac{3}{4} u_{i+1 / 2, j, k}+\frac{1}{4} u_{i+1 / 2, j-1, k}, & \hat{u}_{i+1 / 2, j-1, k} \in(3 / 4,1]
\end{array} ;\right.
$$




$$
\begin{gathered}
- \text { se } \bar{v}_{i+1 / 2, j-1 / 2, k}<0, \text { então } \hat{u}_{i+1 / 2, j, k}=\frac{u_{i+1 / 2, j, k}-u_{i+1 / 2, j+1, k}}{u_{i+1 / 2, j-1, k}-u_{i+1 / 2, j+1, k}}, \\
u_{i+1 / 2, j-1 / 2, k}=\left\{\begin{array}{ll}
u_{i+1 / 2, j, k}, & \hat{u}_{i+1 / 2, j, k} \notin[0,1] \\
\frac{7}{4} u_{i+1 / 2, j, k}-\frac{3}{4} u_{i+1 / 2, j+1, k}, & \hat{u}_{i+1 / 2, j, k} \in[0,3 / 8) \\
\frac{3}{8} u_{i+1 / 2, j-1, k}+\frac{3}{4} u_{i+1 / 2, j, k}-\frac{1}{8} u_{i+1 / 2, j+1, k}, & \hat{u}_{i+1 / 2, j, k} \in[3 / 8,3 / 4] \\
\frac{3}{4} u_{i+1 / 2, j-1, k}+\frac{1}{4} u_{i+1 / 2, j, k}, & \hat{u}_{i+1 / 2, j, k} \in(3 / 4,1]
\end{array} .\right.
\end{gathered}
$$

Considerando-se agora o calculo do termo convectivo $\operatorname{conv}\left(v S^{x x}\right)_{i, j, k}$ na equação (3.21) temos:

$$
\begin{aligned}
\left.\frac{\partial\left(v S^{x x}\right)}{\partial y}\right|_{i, j, k} & \approx \operatorname{conv}\left(v S^{x x}\right)_{i, j, k} \\
& =\frac{\bar{v}_{i, j+1 / 2, k} S_{i, j+1 / 2, k}^{x x}-\bar{v}_{i, j-1 / 2, k} S_{i, j-1 / 2, k}^{x x}}{\delta y}
\end{aligned}
$$

Os valores transportados da componente $S^{x x}$ do tensor não-newtoniano são obtidos pelo método CUBISTA como segue:

- $S_{i, j+1 / 2, k}^{x x}$ :

- se $\bar{v}_{i, j+1 / 2, k} \geq 0$, então $\hat{S}_{i, j, k}^{x x}=\frac{S_{i, j, k}^{x x}-S_{i, j-1, k}^{x x}}{S_{i, j+1, k}^{x x}-S_{i, j-1, k}^{x x}}$,

$$
S_{i, j+1 / 2, k}^{x x}=\left\{\begin{array}{ll}
S_{i, j, k}^{x x}, & \hat{S}_{i, j, k}^{x x} \notin[0,1] \\
\frac{7}{4} S_{i, j, k}^{x x}-\frac{3}{4} S_{i, j-1, k}^{x x}, & \hat{S}_{i, j, k}^{x x} \in[0,3 / 8) \\
\frac{3}{8} S_{i, j+1, k}^{x x}+\frac{3}{4} S_{i, j, k}^{x x}-\frac{1}{8} S_{i, j-1, k}^{x x}, & \hat{S}_{i, j, k}^{x x} \in[3 / 8,3 / 4] \\
\frac{3}{4} S_{i, j+1, k}^{x x}+\frac{1}{4} S_{i, j, k}^{x x}, & \hat{S}_{i, j, k}^{x x} \in(3 / 4,1]
\end{array} ;\right.
$$


- se $\bar{v}_{i, j+1 / 2, k}<0$, então $\hat{S}_{i, j+1, k}^{x x}=\frac{S_{i, j+1, k}^{x x}-S_{i, j+2, k}^{x x}}{S_{i, j, k}^{x x}-S_{i, j+2, k}^{x x}}$

$$
S_{i, j+1 / 2, k}^{x x}=\left\{\begin{array}{ll}
S_{i, j+1, k}^{x x}, & \hat{S}_{i, j+1, k}^{x x} \notin[0,1] \\
\frac{7}{4} S_{i, j+1, k}^{x x}-\frac{3}{4} S_{i, j+2, k}^{x x}, & \hat{S}_{i, j+1, k}^{x x} \in[0,3 / 8) \\
\frac{3}{8} S_{i, j, k}^{x x}+\frac{3}{4} S_{i, j+1, k}^{x x}-\frac{1}{8} S_{i, j+2, k}^{x x}, & \hat{S}_{i, j+1, k}^{x x} \in[3 / 8,3 / 4] \\
\frac{3}{4} S_{i, j, k}^{x x}+\frac{1}{4} S_{i, j+1, k}^{x x}, & \hat{S}_{i, j+1, k}^{x x} \in(3 / 4,1]
\end{array} .\right.
$$

- $S_{i, j-1 / 2, k}^{x x}$ :

- se $\bar{v}_{i, j-1 / 2, k} \geq 0$, então $\hat{S}_{i, j-1, k}^{x x}=\frac{S_{i, j-1, k}^{x x}-S_{i, j-2, k}^{x x}}{S_{i, j, k}^{x x}-S_{i, j-2, k}^{x x}}$,

$$
S_{i, j-1 / 2, k}^{x x}=\left\{\begin{array}{ll}
S_{i, j-1, k}^{x x}, & \hat{S}_{i, j-1, k}^{x x} \notin[0,1] \\
\frac{7}{4} S_{i, j-1, k}^{x x}-\frac{3}{4} S_{i, j-2, k}^{x x}, & \hat{S}_{i, j-1, k}^{x x} \in[0,3 / 8) \\
\frac{3}{8} S_{i, j, k}^{x x}+\frac{3}{4} S_{i, j-1, k}^{x x}-\frac{1}{8} S_{i, j-2, k}^{x x}, & \hat{S}_{i, j-1, k}^{x x} \in[3 / 8,3 / 4] \\
\frac{3}{4} S_{i, j, k}^{x x}+\frac{1}{4} S_{i, j-1, k}^{x x}, & \hat{S}_{i, j-1, k}^{x x} \in(3 / 4,1]
\end{array} ;\right.
$$

- se $\bar{v}_{i, j-1 / 2, k}<0$, então $\bar{v}_{i, j+1 / 2, k}<0$, então $\hat{S}_{i, j, k}^{x x}=\frac{S_{i, j, k}^{x x}-S_{i, j+1, k}^{x x}}{S_{i, j-1, k}^{x x}-S_{i, j+1, k}^{x x}}$

$$
S_{i, j-1 / 2, k}^{x x}=\left\{\begin{array}{ll}
S_{i, j, k}^{x x}, & \hat{S}_{i, j, k}^{x x} \notin[0,1] \\
\frac{7}{4} S_{i, j, k}^{x x}-\frac{3}{4} S_{i, j+1, k}^{x x}, & \hat{S}_{i, j, k}^{x x} \in[0,3 / 8) \\
\frac{3}{8} S_{i, j-1, k}^{x x}+\frac{3}{4} S_{i, j, k}^{x x}-\frac{1}{8} S_{i, j+1, k}^{x x}, & \hat{S}_{i, j, k}^{x x} \in[3 / 8,3 / 4] \\
\frac{3}{4} S_{i, j-1, k}^{x x}+\frac{1}{4} S_{i, j, k}^{x x}, & \hat{S}_{i, j, k}^{x x} \in(3 / 4,1]
\end{array} .\right.
$$

Os demais termos convectivos das equações (3.5) e (3.21), bem como os demais termos convectivos que surgem nas demais componentes da equação da conservação de quantidade de movimento e da equação constitutiva de Maxwell discretizadas são calculados da mesma forma pelo método CUBISTA. 


\subsection{Aproximação das condições de contorno na superfície livre}

Na Seção 1.5, foram obtidas as equações a serem satisfeitas na superfície livre, que repetimos aqui juntamente com a equação de conservação de massa:

$$
\begin{aligned}
& -p+S^{x x} n_{x}^{2}+S^{y y} n_{y}^{2}+S^{z z} n_{z}^{2}+2\left(S^{x y} n_{x} n_{y}+S^{x z} n_{x} n_{z}+S^{y z} n_{y} n_{z}\right) \\
& +\frac{2}{R e}\left[\frac{\partial u}{\partial x} n_{x}^{2}+\frac{\partial v}{\partial y} n_{y}^{2}+\frac{\partial w}{\partial z} n_{z}^{2}+\left(\frac{\partial u}{\partial y}+\frac{\partial v}{\partial x}\right) n_{x} n_{y}\right. \\
& \left.+\left(\frac{\partial u}{\partial z}+\frac{\partial w}{\partial x}\right) n_{x} n_{z}+\left(\frac{\partial v}{\partial z}+\frac{\partial w}{\partial y}\right) n_{y} n_{z}\right]=0 \\
& S^{x x} n_{x} m_{1 x}+S^{y y} n_{y} m_{1 y}+S^{z z} n_{z} m_{1 z}+S^{x y}\left(n_{y} m_{1 x}+n_{x} m_{1 y}\right) \\
& +S^{x z}\left(n_{z} m_{1 x}+n_{x} m_{1 z}\right)+S^{y z}\left(n_{z} m_{1 y}+n_{y} m_{1 z}\right)+\frac{1}{R e}\left[2 \frac{\partial u}{\partial x} n_{x} m_{1 x}\right. \\
& +2 \frac{\partial v}{\partial y} n_{y} m_{1 y}+2 \frac{\partial w}{\partial z} n_{z} m_{1 z}+\left(\frac{\partial u}{\partial y}+\frac{\partial v}{\partial x}\right)\left(n_{y} m_{1 x}+n_{x} m_{1 y}\right) \\
& \left.+\left(\frac{\partial u}{\partial z}+\frac{\partial w}{\partial x}\right)\left(n_{z} m_{1 x}+n_{x} m_{1 z}\right)+\left(\frac{\partial v}{\partial z}+\frac{\partial w}{\partial y}\right)\left(n_{z} m_{1 y}+n_{y} m_{1 z}\right)\right]=0, \\
& S^{x x} n_{x} m_{2 x}+S^{y y} n_{y} m_{2 y}+S^{z z} n_{z} m_{2 z}+S^{x y}\left(n_{y} m_{2 x}+n_{x} m_{2 y}\right) \\
& +S^{x z}\left(n_{z} m_{2 x}+n_{x} m_{2 z}\right)+S^{y z}\left(n_{z} m_{2 y}+n_{y} m_{2 z}\right)+\frac{1}{R e}\left[2 \frac{\partial u}{\partial x} n_{x} m_{2 x}\right. \\
& +2 \frac{\partial v}{\partial y} n_{y} m_{2 y}+2 \frac{\partial w}{\partial z} n_{z} m_{2 z}+\left(\frac{\partial u}{\partial y}+\frac{\partial v}{\partial x}\right)\left(n_{y} m_{2 x}+n_{x} m_{2 y}\right) \\
& \left.+\left(\frac{\partial u}{\partial z}+\frac{\partial w}{\partial x}\right)\left(n_{z} m_{2 x}+n_{x} m_{2 z}\right)+\left(\frac{\partial v}{\partial z}+\frac{\partial w}{\partial y}\right)\left(n_{z} m_{2 y}+n_{y} m_{2 z}\right)\right]=0, \\
& \frac{\partial u}{\partial x}+\frac{\partial v}{\partial y}+\frac{\partial w}{\partial z}=0
\end{aligned}
$$

Para aplicar as condições (3.28)-(3.30) vamos usar as idéias de Tomé et al. [6]. Suponhamos que a malha é suficientemente fina $(\delta x, \delta y, \delta z \ll 1)$ e que localmente a superfície livre pode ser aproximada por uma superfície linear. Nesse caso, podemos considerar três tipos de aproximações para a 
superfície livre conforme especificado abaixo:

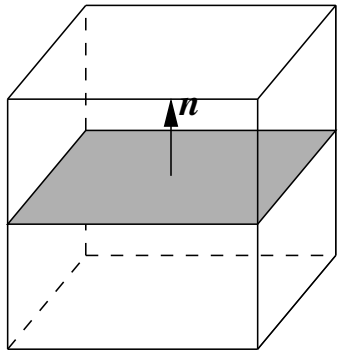

(a)

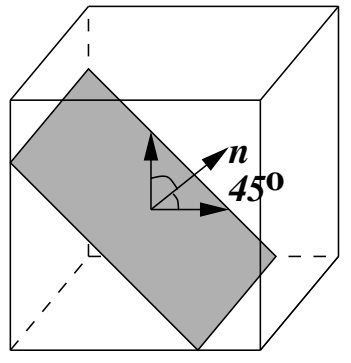

(b)

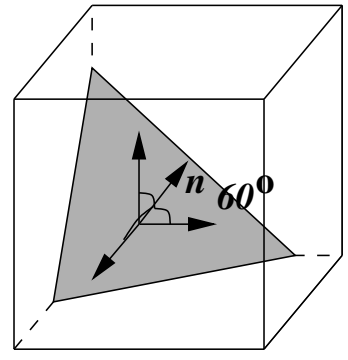

(c)

Figura 3.4: Tipos de superfícies lineares: 1D (a), 2D (b) e 3D (c).

Superfícies 1D: Essas superfícies são definidas por planos perpendiculares a um dos eixos ordenados. Nessas superfícies, o vetor normal toma a forma:

$$
\mathbf{n}=( \pm 1,0,0)^{T}, \quad \mathbf{n}=(0, \pm 1,0)^{T}, \quad \mathbf{n}=(0,0, \pm 1)^{T}
$$

Essas superfícies são identificadas por células $\mathrm{S}$ que possuem somente uma face em contato com face de célula E;

Superfícies 2D: Essas superfícies são definidas por planos que formam um ângulo de $45^{\circ} \mathrm{com}$ dois dos eixos ordenados. Nessas superfícies o vetor normal toma a forma

$\mathbf{n}=( \pm \sqrt{2} / 2, \pm \sqrt{2} / 2,0)^{T}, \mathbf{n}=( \pm \sqrt{2} / 2,0, \pm \sqrt{2} / 2)^{T}, \mathbf{n}=(0, \pm \sqrt{2} / 2, \pm \sqrt{2} / 2)^{T}$

Essas superfícies são identificadas por células $\mathrm{S}$ que possuem somente duas faces adjacentes em contato com faces de células E;

Superfícies 3D: Essas superfícies são compostas por planos que formam um ângulo de $60^{\circ}$ com os três eixos ordenados. Assume-se que nessas superfícies o vetor normal toma a forma

$$
\mathbf{n}=( \pm \sqrt{3} / 3, \pm \sqrt{3} / 3, \pm \sqrt{3} / 3)^{T}
$$


Essas superfícies são identificadas por células S que possuem três faces adjacentes em contato com faces de células E;

Exemplos desses tipos de superfícies são mostrados na figura 3.8.

As equações (3.28)-(3.31) serão aplicadas de acordo com o tipo de superfície. Vamos mostrar como calcular a pressão e as velocidades utilizando as equações (3.28)-(3.31) para um caso particular de cada tipo de superfície. Para os outros casos de superfícies 1D, 2D e 3D, o cálculo da pressão e das velocidades é análogo.

\section{Superfícies 1D}

Considere a célula S mostrada na Figura 3.5.

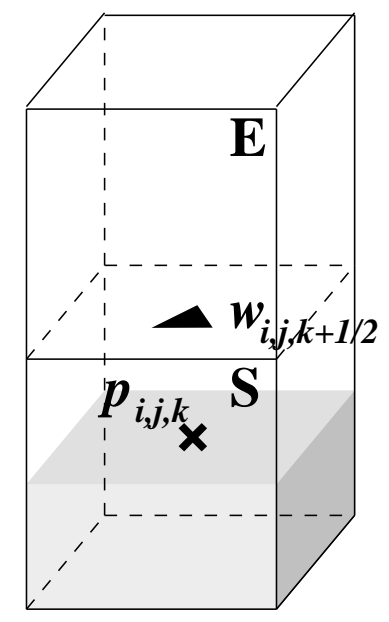

Figura 3.5: Célula S com superfície linear do tipo 1D.

Neste caso, toma-se $\mathbf{n}=(0,0,1)^{T}, \mathbf{m}_{\mathbf{1}}=(1,0,0)^{T}$ e $\mathbf{m}_{\mathbf{2}}=(0,1,0)^{T}$, e as equações (3.28)-(3.30) se reduzem a

$$
\begin{gathered}
-p+S^{z z}+\frac{2}{R e} \frac{\partial w}{\partial z}=0, \\
S^{x z}+\frac{1}{R e}\left(\frac{\partial u}{\partial z}+\frac{\partial w}{\partial x}\right)=0,
\end{gathered}
$$




$$
S^{y z}+\frac{1}{R e}\left(\frac{\partial v}{\partial z}+\frac{\partial w}{\partial y}\right)=0
$$

Os valores de $u_{i+1 / 2, j, k+1}, v_{i, j+1 / 2, k+1}, w_{i, j, k+1 / 2}$ e $p_{i, j, k}$ são necessários quando as velocidades intermediárias $\widetilde{u}_{i+1 / 2, j, k}, \widetilde{v}_{i, j+1 / 2, k}$ e $\widetilde{w}_{i, j, k+1 / 2}$ forem calculadas pelas equações de diferenças correspondentes às equações (3.1)(3.3). Estes valores são obtidos como segue:

Aplicando-se a equação da continuidade (3.31) no centro da célula, vem:

$$
\left(\frac{\partial u}{\partial x}\right)_{i, j, k}+\left(\frac{\partial v}{\partial y}\right)_{i, j, k}+\frac{w_{i, j, k+1 / 2}-w_{i, j, k-1 / 2}}{\delta z}=0
$$

onde

$$
\left(\frac{\partial u}{\partial x}\right)_{i, j, k}=\frac{u_{i+1 / 2, j, k}-u_{i-1 / 2, j, k}}{\delta x}, \quad\left(\frac{\partial v}{\partial y}\right)_{i, j, k}=\frac{v_{i, j+1 / 2, k}-v_{i, j-1 / 2, k}}{\delta y} .
$$

Portanto,

$$
w_{i, j, k+1 / 2}=w_{i, j, k-1 / 2}-\delta z\left[\left(\frac{\partial u}{\partial x}\right)_{i, j, k}+\left(\frac{\partial v}{\partial y}\right)_{i, j, k}\right]
$$

Agora, discretizando (3.33) na posição $(i+1 / 2, j, k+1 / 2)$ tem-se:

$$
S_{i+1 / 2, j, k+1 / 2}^{x z}+\frac{1}{R e}\left[\frac{u_{i+1 / 2, j, k+1}-u_{i+1 / 2, j, k}}{\delta z}+\left(\frac{\partial w}{\partial x}\right)_{i+1 / 2, j, k+1 / 2}\right]=0 .
$$

Explicitando $u_{i+1 / 2, j, k+1}$ em (3.37) obtemos

$$
u_{i+1 / 2, j, k+1}=u_{i+1 / 2, j, k}-\delta z\left(\frac{\partial w}{\partial x}\right)_{i+1 / 2, j, k+1 / 2}-\operatorname{Re} \delta z S_{i+1 / 2, j, k+1 / 2}^{x z}
$$

onde

$$
\left(\frac{\partial w}{\partial x}\right)_{i+1 / 2, j, k+1 / 2}=\frac{w_{i+1, j, k+1 / 2}-w_{i, j, k+1 / 2}}{\delta x} .
$$

Da mesma forma, discretizando a equação (3.34) na posição $(i, j+1 / 2, k+$ 
$1 / 2$ ) e isolando $v_{i, j+1 / 2, k+1}$ obtém-se

$$
v_{i, j+1 / 2, k+1}=v_{i, j+1 / 2, k}-\delta z\left(\frac{\partial w}{\partial y}\right)_{i, j+1 / 2, k+1 / 2}-\operatorname{Re} \delta z S_{i, j+1 / 2, k+1 / 2}^{y z}
$$

onde

$$
\left(\frac{\partial w}{\partial y}\right)_{i, j+1 / 2, k+1 / 2}=\frac{w_{i, j+1, k+1 / 2}-w_{i, j, k+1 / 2}}{\delta y} .
$$

Tendo obtido as velocidades $u_{i+1 / 2, j, k+1}, v_{i, j+1 / 2, k+1}$ e $w_{i, j, k+1 / 2}$, a pressão $p_{i, j, k}$ é obtida através da equação (3.32) aplicada no centro da célula, ou seja,

$$
p_{i, j, k}=S_{i, j, k}^{z z}+\frac{2}{R e}\left(\frac{w_{i, j, k+1 / 2}-w_{i, j, k-1 / 2}}{\delta z}\right) .
$$

\section{Superfícies 2D}

Considere a célula $\mathrm{S}$ mostrada na Figura 3.6. Neste caso, toma-se $\mathbf{n}=$ $(\sqrt{2} / 2,0, \sqrt{2} / 2)^{T}, \mathbf{m}_{\mathbf{1}}=(\sqrt{2} / 2,0,-\sqrt{2} / 2)^{T}$ e $\mathbf{m}_{\mathbf{2}}=(0,1,0)^{T}$, e as equações (3.28) e (3.29) se reduzem a

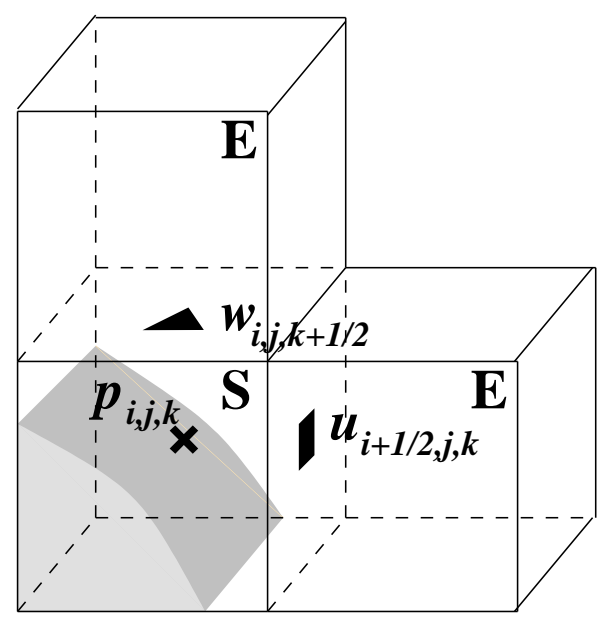

Figura 3.6: Célula S com superfície linear do tipo 2D. 


$$
\begin{gathered}
-p+\frac{1}{2} S^{x x}+\frac{1}{2} S^{z z}+S^{x z}+\frac{1}{R e}\left(\frac{\partial u}{\partial x}+\frac{\partial w}{\partial z}+\frac{\partial u}{\partial z}+\frac{\partial w}{\partial x}\right)=0, \\
\frac{1}{2} S^{x x}-\frac{1}{2} S^{z z}+\frac{1}{R e}\left(\frac{\partial u}{\partial x}-\frac{\partial w}{\partial z}\right)=0 .
\end{gathered}
$$

As velocidades $u_{i+1 / 2, j, k}$ e $w_{i, j, k+1 / 2}$ são necessárias e devem ser calculadas aplicando-se a equação da continuidade (3.31) e a equação (3.44) no centro da célula. Neste caso, tem-se

$$
\frac{u_{i+1 / 2, j, k}-u_{i-1 / 2, j, k}}{\delta x}+\left(\frac{\partial v}{\partial y}\right)_{i, j, k}+\frac{w_{i, j, k+1 / 2}-w_{i, j, k-1 / 2}}{\delta z}=0 .
$$

Isolando-se $u_{i+1 / 2, j, k}$ e $w_{i, j, k+1 / 2}$, vem:

$$
u_{i+1 / 2, j, k}+\frac{\delta x}{\delta z} w_{i, j, k+1 / 2}=u_{i-1 / 2, j, k}+\frac{\delta x}{\delta z} w_{i, j, k-1 / 2}-\delta x\left(\frac{\partial v}{\partial y}\right)_{i, j, k}
$$

Agora, aplicando (3.44) no centro da célula, obtém-se

$$
\frac{u_{i+1 / 2, j, k}-u_{i-1 / 2, j, k}}{\delta x}-\frac{w_{i, j, k+1 / 2}-w_{i, j, k-1 / 2}}{\delta z}+\frac{R e}{2}\left(S_{i, j, k}^{x x}-S_{i, j, k}^{z z}\right)=0,
$$

e isolando-se $u_{i+1 / 2, j, k}$ e $w_{i, j, k+1 / 2}$, tem-se

$$
u_{i+1 / 2, j, k}-\frac{\delta x}{\delta z} w_{i, j, k+1 / 2}=u_{i-1 / 2, j, k}-\frac{\delta x}{\delta z} w_{i, j, k-1 / 2}-\frac{R e \delta x}{2}\left(S_{i, j, k}^{x x}-S_{i, j, k}^{z z}\right) .
$$

Das equações (3.46) e (3.48), temos que

$$
\begin{aligned}
& u_{i+1 / 2, j, k}=u_{i-1 / 2, j, k}-\frac{\delta x}{2}\left(\frac{\partial v}{\partial y}\right)_{i, j, k}-\frac{\operatorname{Re} \delta x}{4}\left(S_{i, j, k}^{x x}-S_{i, j, k}^{z z}\right) \\
& w_{i, j, k+1 / 2}=w_{i, j, k-1 / 2}-\frac{\delta z}{2}\left(\frac{\partial v}{\partial y}\right)_{i, j, k}+\frac{\operatorname{Re} \delta z}{4}\left(S_{i, j, k}^{x x}-S_{i, j, k}^{z z}\right) .
\end{aligned}
$$

Tendo calculado $u_{i+1 / 2, j, k}$ e $w_{i, j, k+1 / 2}$, a pressão é obtida por meio da equação 
(3.43) aplicada no centro da célula, ou seja:

$$
\begin{aligned}
p_{i, j, k}= & \frac{1}{2} S_{i, j, k}^{x x}+\frac{1}{2} S_{i, j, k}^{z z}+S_{i, j, k}^{x z} \\
& +\frac{1}{R e}\left[\left(\frac{\partial u}{\partial x}\right)_{i, j, k}+\left(\frac{\partial w}{\partial z}\right)_{i, j, k}+\left(\frac{\partial u}{\partial z}\right)_{i, j, k}+\left(\frac{\partial w}{\partial x}\right)_{i, j, k}\right] .
\end{aligned}
$$

\section{Superfícies 3D}

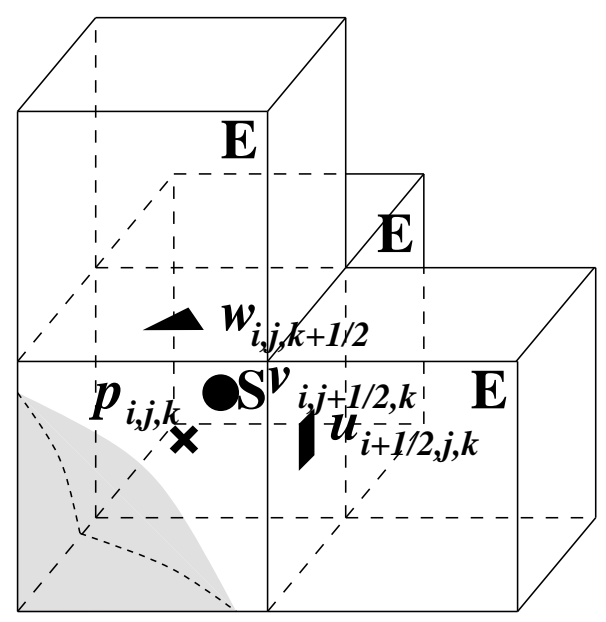

Figura 3.7: Célula S com superfície linear do tipo 3D.

Considere a célula $\mathrm{S}$ com três faces adjacentes em contato com faces de células E como mostrada na Figura 3.7. Nesse caso, tomamos $\mathbf{n}=$ $(\sqrt{3} / 3, \sqrt{3} / 3, \sqrt{3} / 3)^{T}, \mathbf{m}_{\mathbf{1}}=(\sqrt{2} / 2,-\sqrt{2} / 2,0)^{T}$ e $\mathbf{m}_{\mathbf{2}}=(\sqrt{6} / 6, \sqrt{6} / 6,-\sqrt{6} / 3)^{T}$, e as equações (3.28)-(3.30) se reduzem a

$$
\begin{gathered}
-p+\frac{1}{3}\left[S^{x x}+S^{y y}+S^{z z}+2\left(S^{x y}+S^{x z}+S^{y z}\right)\right] \\
+\frac{2 R e}{3}\left(\frac{\partial u}{\partial y}+\frac{\partial v}{\partial x}+\frac{\partial u}{\partial z}+\frac{\partial w}{\partial x}+\frac{\partial v}{\partial z}+\frac{\partial w}{\partial y}\right)=0, \\
S^{x x}-S^{y y}+S^{x z}-S^{y z} \\
+\frac{1}{R e}\left(2 \frac{\partial u}{\partial x}-2 \frac{\partial v}{\partial y}+\frac{\partial u}{\partial z}+\frac{\partial w}{\partial x}-\frac{\partial v}{\partial z}-\frac{\partial w}{\partial y}\right)=0,
\end{gathered}
$$




$$
\begin{aligned}
& S^{x x}+S^{y y}-2 S^{z z}+2 S^{x y}-S^{x z}-S^{y z} \\
& +\frac{1}{R e}\left(2 \frac{\partial u}{\partial x}+2 \frac{\partial v}{\partial y}-4 \frac{\partial w}{\partial z}+2 \frac{\partial u}{\partial y}+2 \frac{\partial v}{\partial x}-\frac{\partial u}{\partial z}-\frac{\partial w}{\partial x}-\frac{\partial v}{\partial z}-\frac{\partial w}{\partial y}\right)=0 .
\end{aligned}
$$

Observamos que a equação da continuidade (3.31) foi utilizada na obtenção da equação (3.52). Entretanto, é necessário determinar as velocidades $u_{i+1 / 2, j, k}, v_{i, j+1 / 2, k}$ e $w_{i, j, k+1 / 2}$, de modo que a equação da continuidade (3.31) será utilizada. Ainda, somando-se as equações (3.53) e (3.54) e dividindo-se o resultado por dois, obtém-se

$$
\begin{aligned}
& S^{x x}-S^{z z}+S^{x y}-S^{y z} \\
& +\frac{1}{R e}\left(2 \frac{\partial u}{\partial x}-2 \frac{\partial w}{\partial z}+\frac{\partial u}{\partial y}+\frac{\partial v}{\partial x}-\frac{\partial v}{\partial z}-\frac{\partial w}{\partial y}\right)=0 .
\end{aligned}
$$

Para obtenção de $u_{i+1 / 2, j, k}, v_{i, j+1 / 2, k}$ e $w_{i, j, k+1 / 2}$, é necessário resolver um sistema linear $3 \times 3$ obtido pelas discretizações da equação da continuidade (3.31) e das equações (3.53) e (3.55) aplicadas no centro da célula S. Esse sistema é então resolvido pelo método de eliminação de Gauss com pivotamento parcial. Maiores detalhes sobre a construção desse sistema linear podem ser visto em Tomé et al. [6].

Tendo obtido $u_{i+1 / 2, j, k}, v_{i, j+1 / 2, k}$ e $w_{i, j, k+1 / 2}$, a pressão é calculada no centro da célula $(i, j, k)$ por meio da equação (3.52), ou seja,

$$
\begin{aligned}
& p_{i, j, k}=\frac{1}{3}\left[S_{i, j, k}^{x x}+S_{i, j, k}^{y y}+S_{i, j, k}^{z z}+2\left(S_{i, j, k}^{x y}+S_{i, j, k}^{x z}+S_{i, j, k}^{y z}\right)\right] \\
& +\frac{2 R e}{3}\left[\left(\frac{\partial u}{\partial y}\right)_{i, j, k}+\left(\frac{\partial v}{\partial x}\right)_{i, j, k}+\left(\frac{\partial u}{\partial z}\right)_{i, j, k}+\left(\frac{\partial w}{\partial x}\right)_{i, j, k}+\left(\frac{\partial v}{\partial z}\right)_{i, j, k}+\left(\frac{\partial w}{\partial y}\right)_{i, j, k}\right]
\end{aligned}
$$

\subsection{Aproximação do tensor não-newtoniano $\mathrm{S}$ em contornos rígidos}

Para a obtenção das componentes do tensor não-newtoniano nos contornos rígidos, utilizam-se as equações obtidas na Seção 1.4. Para esse fim, percorrese as células de contorno (B) e verifica se a célula está em contato com células cheias (F) ou de superfície (S). 
Nas equações que se seguem, as derivadas são aproximadas como nas seções anteriores ao passo que os sobre-indices $n+1$ e $n$ denotam os tempos $t_{n}+\delta t$ e $t_{n}$, respectivamente.

\subsubsection{Contornos paralelos ao plano $x y$}

Esses contornos são identificados por células B com apenas a face $(k+1 / 2)$ ou $(k-1 / 2)$ em contato com uma face de células $\mathrm{F}$ ou $\mathrm{S}$ conforme mostrado na figura 3.8 .

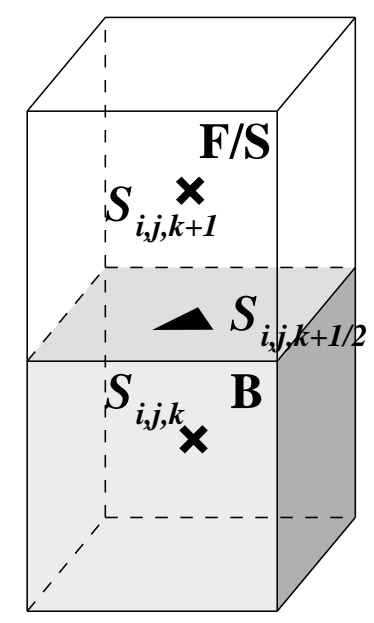

(a)

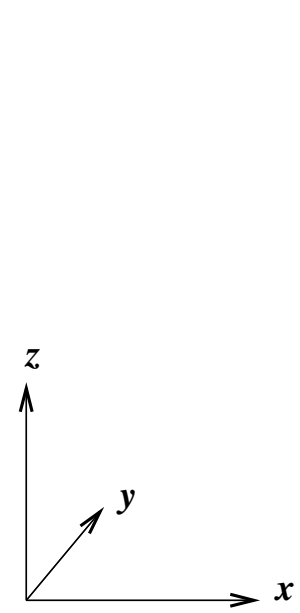

$x$

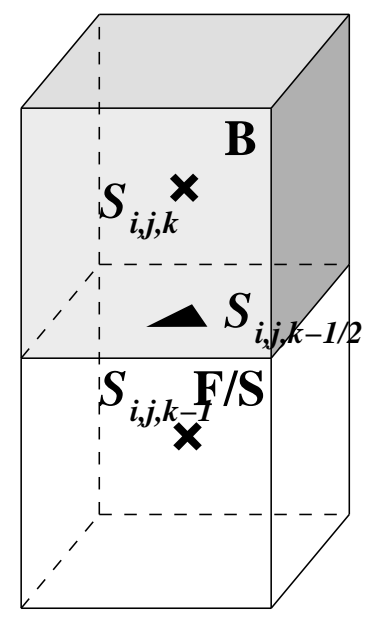

(b)

Figura 3.8: Configurações de células B com apenas a face $(k+1 / 2)$ (a) ou apenas a face $(k-1 / 2)$ (b) em contato com célula $\mathrm{F}$ ou $\mathrm{S}$.

Considerando a célula B mostrada na figura 3.8(a), os valores das componentes do tensor $\mathbf{S}$ são obtidas por meio das equações (1.51), (1.62), (1.63), (1.66), (1.67) e (1.68) que são aproximadas na posição $(i, j, k+1 / 2)$ como segue:

$$
\begin{gathered}
\left(S^{z z}\right)_{i, j, k+1 / 2}^{n+1}=0 \\
\left(S^{x z}\right)_{i, j, k+1 / 2}^{n+1}=e^{-\frac{1}{W e} \delta t}\left(S^{x z}\right)_{i, j, k+1 / 2}^{n} \\
+\frac{1}{2 R e}\left(e^{-\frac{1}{W e} \delta t}+1\right)\left(\left(\frac{\partial u}{\partial z}\right)_{i, j, k+1 / 2}^{n}-\left(\frac{\partial u}{\partial z}\right)_{i, j, k+1 / 2}^{n+1}\right)
\end{gathered}
$$




$$
\begin{aligned}
& \left(S^{y z}\right)_{i, j, k+1 / 2}^{n+1}=e^{-\frac{1}{W e} \delta t}\left(S^{y z}\right)_{i, j, k+1 / 2}^{n} \\
& +\frac{1}{2 R e}\left(e^{-\frac{1}{W e} \delta t}+1\right)\left(\left(\frac{\partial v}{\partial z}\right)_{i, j, k+1 / 2}^{n}-\left(\frac{\partial v}{\partial z}\right)_{i, j, k+1 / 2}^{n+1}\right) \\
& \left(S^{x x}\right)_{i, j, k+1 / 2}^{n+1}=e^{-\frac{1}{W e} \delta t}\left(S^{x x}\right)_{i, j, k+1 / 2}^{n} \\
& +\delta t\left(\left(\frac{\partial u}{\partial z}\right)_{i, j, k+1 / 2}^{n+1}\left(S^{x z}\right)_{i, j, k+1 / 2}^{n+1}+\left(\frac{\partial u}{\partial z}\right)_{i, j, k+1 / 2}^{n} e^{-\frac{1}{W e} \delta t}\left(S^{x z}\right)_{i, j, k+1 / 2}^{n}\right) \\
& +\frac{1}{2} \frac{W e}{R e}\left(\left(\frac{\partial u}{\partial z}\right)_{i, j, k+1 / 2}^{n}+\left(\frac{\partial u}{\partial z}\right)_{i, j, k+1 / 2}^{n+1}\right)^{2}\left(1-e^{-\frac{1}{W e} \delta t}\right) \\
& \left(S^{y y}\right)_{i, j, k+1 / 2}^{n+1}=e^{-\frac{1}{W e} \delta t}\left(S^{y y}\right)_{i, j, k+1 / 2}^{n} \\
& +\delta t\left(\left(\frac{\partial v}{\partial z}\right)_{i, j, k+1 / 2}^{n+1}\left(S^{y z}\right)_{i, j, k+1 / 2}^{n+1}+\left(\frac{\partial v}{\partial z}\right)_{i, j, k+1 / 2}^{n} e^{-\frac{1}{W e} \delta t}\left(S^{y z}\right)_{i, j, k+1 / 2}^{n}\right) \\
& +\frac{1}{2} \frac{W e}{R e}\left(\left(\frac{\partial v}{\partial z}\right)_{i, j, k+1 / 2}^{n}+\left(\frac{\partial v}{\partial z}\right)_{i, j, k+1 / 2}^{n+1}\right)^{2}\left(1-e^{-\frac{1}{W e} \delta t}\right) \\
& \left(S^{x y}\right)_{i, j, k+1 / 2}^{n+1}=e^{-\frac{1}{W e} \delta t}\left(S^{x y}\right)_{i, j, k+1 / 2}^{n} \\
& +\frac{\delta t}{2}\left(\left(\frac{\partial u}{\partial z}\right)_{i, j, k+1 / 2}^{n+1}\left(S^{y z}\right)_{i, j, k+1 / 2}^{n+1}+\left(\frac{\partial u}{\partial z}\right)_{i, j, k+1 / 2}^{n} e^{-\frac{1}{W e} \delta t}\left(S^{y z}\right)_{i, j, k+1 / 2}^{n}\right) \\
& +\frac{\delta t}{2}\left(\left(\frac{\partial v}{\partial z}\right)_{i, j, k+1 / 2}^{n+1}\left(S^{x z}\right)_{i, j, k+1 / 2}^{n+1}+\left(\frac{\partial v}{\partial z}\right)_{i, j, k+1 / 2}^{n} e^{-\frac{1}{W e} \delta t}\left(S^{x z}\right)_{i, j, k+1 / 2}^{n}\right) \\
& +\frac{1}{2} \frac{W e}{R e}\left(\left(\frac{\partial u}{\partial z}\right)_{i, j, k+1 / 2}^{n}+\left(\frac{\partial u}{\partial z}\right)_{i, j, k+1 / 2}^{n+1}\right)\left(\left(\frac{\partial v}{\partial z}\right)_{i, j, k+1 / 2}^{n}+\left(\frac{\partial v}{\partial z}\right)_{i, j, k+1 / 2}^{n+1}\right)\left(1-e^{-\frac{1}{W e} \delta t}\right) .
\end{aligned}
$$

Tendo obtido os valores das componentes do contorno na posição $(i, j, k+$ $1 / 2$ ), os valores do tensor $\mathbf{S}$ no centro da célula B são obtidos por extrapolação 
linear usando os valores em $(i, j, k+1)$ e $(i, j, k+1 / 2)$, obtendo-se

$$
\mathbf{S}_{i, j, k}^{n+1}=2 \mathbf{S}_{i, j, k+1 / 2}^{n+1}-\mathbf{S}_{i, j, k+1}^{n+1}
$$

Caso a célula F ou S em contato seja como na figura 3.8(b), as equações (1.51), (1.62), (1.63), (1.66), (1.67) e (1.68) são aproximadas de forma análoga no ponto $(i, j, k-1 / 2)$, e as componentes do tensor $\mathbf{S}$ em $(i, j, k)$ são obtidas ao fazer

$$
\mathbf{S}_{i, j, k}^{n+1}=2 \mathbf{S}_{i, j, k-1 / 2}^{n+1}-\mathbf{S}_{i, j, k-1}^{n+1}
$$

\subsubsection{Contornos paralelos ao plano $x z$}

Esses contornos são identificados por células B com apenas a face $(j+1 / 2)$ ou $(j-1 / 2)$ em contato com uma face de células $\mathrm{F}$ ou $\mathrm{S}$ conforme mostrado na figura 3.9.

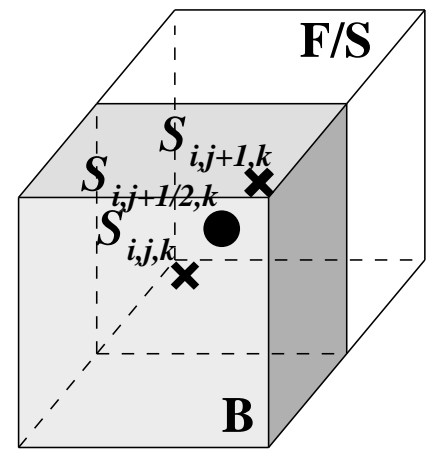

(a)

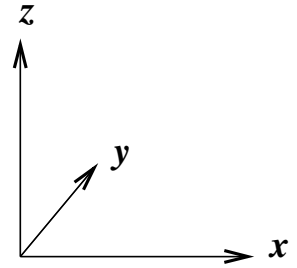

r'

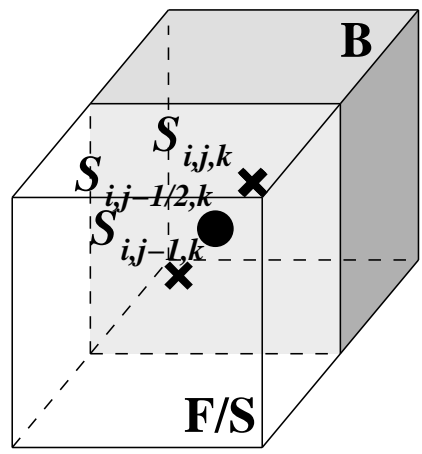

(b)

Figura 3.9: Configurações de células B com apenas a face $(j+1 / 2)$ (a) ou apenas a face $(j-1 / 2)$ (b) em contato com uma face da célula $\mathrm{F}$ ou $\mathrm{S}$.

Considerando a célula B como mostrado na figura 3.9(a), o tensor $\mathbf{S}$ é calculado pelas equações (1.75)-(1.80) aproximadas na posição $(i, j+1 / 2, k)$, que fornecem, respectivamente,

$$
\left(S^{y y}\right)_{i, j+1 / 2, k}^{n+1}=0
$$




$$
\begin{aligned}
& \left(S^{x y}\right)_{i, j+1 / 2, k}^{n+1}=e^{-\frac{1}{W e} \delta t}\left(S^{x y}\right)_{i, j+1 / 2, k}^{n} \\
& +\frac{1}{2 R e}\left(e^{-\frac{1}{W e} \delta t}+1\right)\left(\left(\frac{\partial u}{\partial y}\right)_{i, j+1 / 2, k}^{n}-\left(\frac{\partial u}{\partial y}\right)_{i, j+1 / 2, k}^{n+1}\right), \\
& \left(S^{y z}\right)_{i, j+1 / 2, k}^{n+1}=e^{-\frac{1}{W e} \delta t}\left(S^{y z}\right)_{i, j+1 / 2, k}^{n} \\
& +\frac{1}{2 R e}\left(e^{-\frac{1}{W e} \delta t}+1\right)\left(\left(\frac{\partial w}{\partial y}\right)_{i, j+1 / 2, k}^{n}-\left(\frac{\partial w}{\partial y}\right)_{i, j+1 / 2, k}^{n+1}\right), \\
& \left(S^{x x}\right)_{i, j+1 / 2, k}^{n+1}=e^{-\frac{1}{W e} \delta t}\left(S^{x x}\right)_{i, j+1 / 2, k}^{n} \\
& +\delta t\left(\left(\frac{\partial u}{\partial y}\right)_{i, j+1 / 2, k}^{n+1}\left(S^{x y}\right)_{i, j+1 / 2, k}^{n+1}+\left(\frac{\partial u}{\partial y}\right)_{i, j+1 / 2, k}^{n} e^{-\frac{1}{W e} \delta t}\left(S^{x y}\right)_{i, j+1 / 2, k}^{n}\right) \\
& +\frac{1}{2} \frac{W e}{R e}\left(\left(\frac{\partial u}{\partial y}\right)_{i, j+1 / 2, k}^{n}+\left(\frac{\partial u}{\partial y}\right)_{i, j+1 / 2, k}^{n+1}\right)^{2}\left(1-e^{-\frac{1}{W e} \delta t}\right), \\
& \left(S^{z z}\right)_{i, j+1 / 2, k}^{n+1}=e^{-\frac{1}{W e} \delta t}\left(S^{z z}\right)_{i, j+1 / 2, k}^{n} \\
& +\delta t\left(\left(\frac{\partial w}{\partial y}\right)_{i, j+1 / 2, k}^{n+1}\left(S^{y z}\right)_{i, j+1 / 2, k}^{n+1}+\left(\frac{\partial w}{\partial y}\right)_{i, j+1 / 2, k}^{n} e^{-\frac{1}{W e} \delta t}\left(S^{y z}\right)_{i, j+1 / 2, k}^{n}\right) \\
& +\frac{1}{2} \frac{W e}{R e}\left(\left(\frac{\partial w}{\partial y}\right)_{i, j+1 / 2, k}^{n}+\left(\frac{\partial w}{\partial y}\right)_{i, j+1 / 2, k}^{n+1}\right)^{2}\left(1-e^{-\frac{1}{W e} \delta t}\right)
\end{aligned}
$$


Tendo obtido os valores das componentes do contorno no ponto $(i, j+$ $1 / 2, k)$, os valores em $(i, j, k)$, centro da célula $\mathrm{B}$, são obtidos por extrapolação linear entre os valores em $(i, j+1, k)$ e $(i, j+1 / 2, k)$, obtendo-se

$$
\mathbf{S}_{i, j, k}^{n+1}=2 \mathbf{S}_{i, j+1 / 2, k}^{n+1}-\mathbf{S}_{i, j+1, k}^{n+1} .
$$

Caso a célula $\mathrm{F}$ ou $\mathrm{S}$ em contato seja como na figura 3.9(b), as equações (1.75)-(1.80) são aproximadas de forma análoga no ponto $(i, j-1 / 2, k)$ e as componentes de $\mathbf{S}$ em $(i, j, k)$ são obtidas ao fazer

$$
\mathbf{S}_{i, j, k}^{n+1}=2 \mathbf{S}_{i, j-1 / 2, k}^{n+1}-\mathbf{S}_{i, j-1, k}^{n+1} .
$$

\subsubsection{Contornos paralelos ao plano $y z$}

Esses contornos são identificados por células B com apenas a face $(i+1 / 2)$ ou $(i-1 / 2)$ em contato com uma face de células $\mathrm{F}$ ou $\mathrm{S}$ conforme mostrado na figura 3.10 .

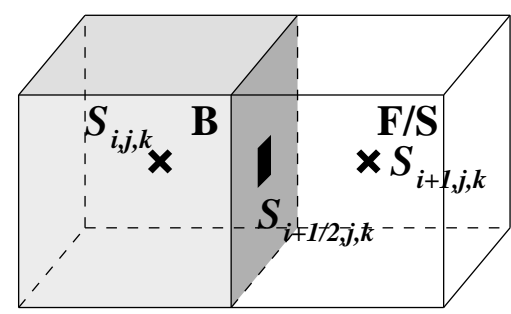

(a)

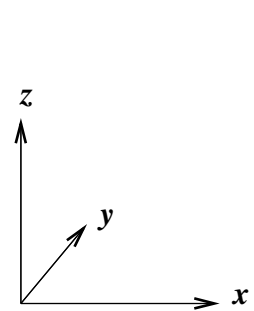

$x$

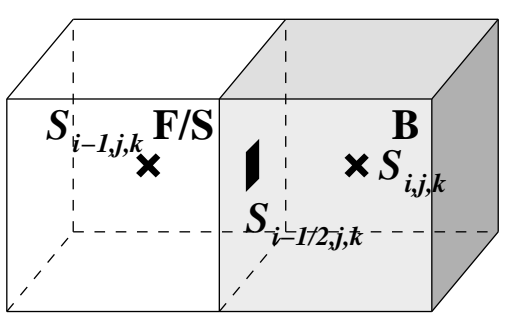

(b)

Figura 3.10: Configurações de células B com apenas a face $(i+1 / 2)$ (a) ou apenas a face $(i-1 / 2)$ (b) em contato com célula $\mathrm{F}$ ou $\mathrm{S}$.

Considerando a célula B como mostrado na figura 3.10(a), as componentes do tensor $\mathbf{S}$ são calculadas pelas equações (1.87)-(1.92) que são aproximadas no ponto $(i+1 / 2, j, k)$, respectivamente, por

$$
\left(S^{x x}\right)_{i+1 / 2, j, k}^{n+1}=0
$$




$$
\begin{aligned}
& \left(S^{x y}\right)_{i+1 / 2, j, k}^{n+1}=e^{-\frac{1}{W e} \delta t}\left(S^{x y}\right)_{i+1 / 2, j, k}^{n} \\
& +\frac{1}{2 R e}\left(e^{-\frac{1}{W e} \delta t}+1\right)\left(\left(\frac{\partial v}{\partial x}\right)_{i+1 / 2, j, k}^{n}-\left(\frac{\partial v}{\partial x}\right)_{i+1 / 2, j, k}^{n+1}\right), \\
& \left(S^{x z}\right)_{i+1 / 2, j, k}^{n+1}=e^{-\frac{1}{W e} \delta t}\left(S^{x z}\right)_{i+1 / 2, j, k}^{n} \\
& +\frac{1}{2 R e}\left(e^{-\frac{1}{W e} \delta t}+1\right)\left(\left(\frac{\partial w}{\partial x}\right)_{i+1 / 2, j, k}^{n}-\left(\frac{\partial w}{\partial x}\right)_{i+1 / 2, j, k}^{n+1}\right), \\
& \left(S^{y y}\right)_{i+1 / 2, j, k}^{n+1}=e^{-\frac{1}{W e} \delta t}\left(S^{y y}\right)_{i+1 / 2, j, k}^{n} \\
& +\delta t\left(\left(\frac{\partial v}{\partial x}\right)_{i+1 / 2, j, k}^{n+1}\left(S^{x y}\right)_{i+1 / 2, j, k}^{n+1}+\left(\frac{\partial v}{\partial x}\right)_{i+1 / 2, j, k}^{n} e^{-\frac{1}{W e} \delta t}\left(S^{x y}\right)_{i+1 / 2, j, k}^{n}\right) \\
& +\frac{1}{2} \frac{W e}{R e}\left(\left(\frac{\partial v}{\partial x}\right)_{i+1 / 2, j, k}^{n}+\left(\frac{\partial v}{\partial x}\right)_{i+1 / 2, j, k}^{n+1}\right)^{2}\left(1-e^{-\frac{1}{W e} \delta t}\right) \\
& \left(S^{z z}\right)_{i+1 / 2, j, k}^{n+1}=e^{-\frac{1}{W e} \delta t}\left(S^{z z}\right)_{i+1 / 2, j, k}^{n} \\
& +\delta t\left(\left(\frac{\partial w}{\partial x}\right)_{i+1 / 2, j, k}^{n+1}\left(S^{x z}\right)_{i+1 / 2, j, k}^{n+1}+\left(\frac{\partial w}{\partial x}\right)_{i+1 / 2, j, k}^{n} e^{-\frac{1}{W e} \delta t}\left(S^{x z}\right)_{i+1 / 2, j, k}^{n}\right) \\
& +\frac{1}{2} \frac{W e}{R e}\left(\left(\frac{\partial w}{\partial x}\right)_{i+1 / 2, j, k}^{n}+\left(\frac{\partial w}{\partial x}\right)_{i+1 / 2, j, k}^{n+1}\right)^{2}\left(1-e^{-\frac{1}{W e} \delta t}\right),
\end{aligned}
$$


Tendo obtido os valores das componentes do contorno no ponto $(i+$ $1 / 2, j, k)$, os valores em $(i, j, k)$, centro da célula $\mathrm{B}$, são obtidos por extrapolação linear entre os valores em $(i+1, j, k)$ e $(i+1 / 2, j, k)$, obtendo-se

$$
\mathbf{S}_{i, j, k}^{n+1}=2 \mathbf{S}_{i+1 / 2, j, k}^{n+1}-\mathbf{S}_{i+1, j, k}^{n+1}
$$

Caso a célula F ou S em contato seja como na figura 3.10(b), as equações (1.87)-(1.92) são aproximadas de forma análoga no ponto $(i-1 / 2, j, k)$, e as componentes de $\mathbf{S}$ em $(i, j, k)$ são obtidas ao fazer

$$
\mathbf{S}_{i, j, k}^{n+1}=2 \mathbf{S}_{i-1 / 2, j, k}^{n+1}-\mathbf{S}_{i-1, j, k}^{n+1}
$$

\subsubsection{Células de canto}

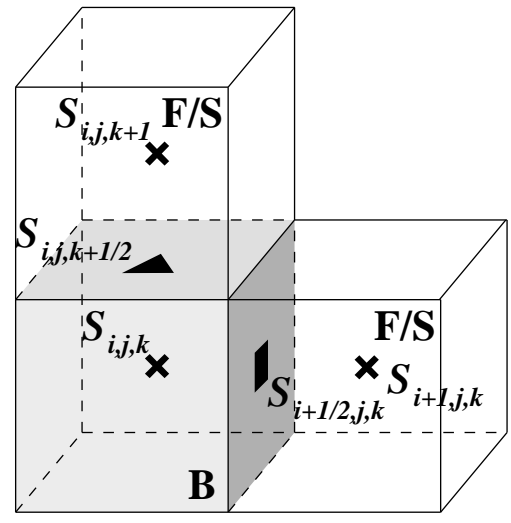

(a)

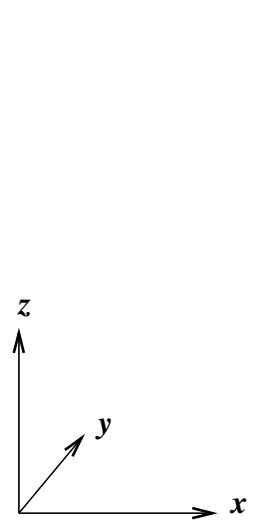

$x$

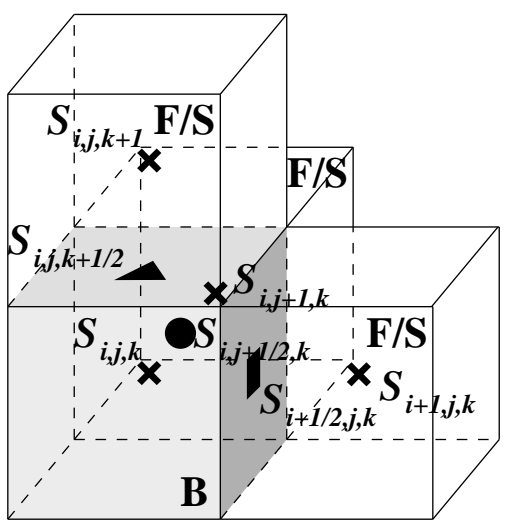

(b)

Figura 3.11: Configurações de células B com duas (a) ou três faces (b) em contato com células F ou S.

Pode ocorrer que o contorno mude de direção abruptamente (como ocorre, por exemplo, na simulação do escoamento em uma contração) e nesse caso pode ocorrer o aparecimento de células B com duas ou três faces adjacentes em contato com células F ou S. Para essas células, primeiramente calcula-se o tensor $\mathbf{S}$ nas faces da célula $\mathrm{B}$ em contato com células $\mathrm{F}$ ou $\mathrm{S}$ (ver seções 3.9.1-3.9.3). O valor das componentes do tensor $\mathbf{S}$ no centro da célula $\mathrm{B}$ 
é obtido fazendo-se a média aritmética entre as direções consideradas. Por exemplo, se considerarmos a célula B mostrada na figura 3.11(a), calcula-se as componentes do tensor $\mathbf{S}$ nos pontos $(i+1 / 2, j, k)$ (ver equações 3.73-3.78) e $(i, j, k+1 / 2)$ (ver equações 3.57-3.62). O tensor $\mathbf{S}$ no centro da célula B é obtido fazendo a média aritmética das equações (3.79) e (3.63), ou seja,

$$
\mathbf{S}_{i, j, k}=\frac{1}{2}\left[\left(2 \mathbf{S}_{i+1 / 2, j, k}-\mathbf{S}_{i+1, j, k}\right)+\left(2 \mathbf{S}_{i, j, k+1 / 2}-\mathbf{S}_{i, j, k+1}\right)\right] .
$$

Existem doze configurações de células B com duas faces em contato com células $\mathrm{F}$ ou $\mathrm{S}$ e o cálculo do tensor $\mathbf{S}$ nessas células é efetuado como descrito acima.

Para células $\mathrm{B}$ com três faces em contato com células $\mathrm{F}$ ou $\mathrm{S}$, o cálculo do tensor $\mathbf{S}$ no centro da célula B é análogo ao caso de células $\mathrm{B}$ com duas faces em contato com células F ou S. Por exemplo, para a célula B mostrada na Figura 3.11(b), calcula-se as componentes de $\mathbf{S}$ nos pontos $(i+1 / 2, j, k)$, $(i, j+1 / 2, k)$ e $(i, j, k+1 / 2)$, e $\mathbf{S}_{i, j, k}$ é dado pela média aritmética de (3.79), (3.71) e (3.63), ou seja,

$$
\begin{aligned}
\mathbf{S}_{i, j, k}= & \frac{1}{3}\left[\left(2 \mathbf{S}_{i+1 / 2, j, k}-\mathbf{S}_{i+1, j, k}\right)+\left(2 \mathbf{S}_{i, j+1 / 2, k}-\mathbf{S}_{i, j+1, k}\right)\right. \\
& \left.+\left(2 \mathbf{S}_{i, j, k+1 / 2}-\mathbf{S}_{i, j, k+1}\right)\right] .
\end{aligned}
$$

As outras sete combinações possíveis de células B com três faces em contato com células F ou S são tratadas de maneira similar.

\subsubsection{Aproximação das derivadas das velocidades}

Nas equações (3.57)-(3.62), (3.65)-(3.70) e (3.73)-(3.78), as derivadas das velocidades são aproximadas como segue:

Considere a derivada $\left(\frac{\partial u}{\partial z}\right)_{i, j, k+1 / 2}^{n}$ que aparece nas equações (3.57)-(3.62). Aproxima-se esta derivada fazendo

$$
\left(\frac{\partial u}{\partial z}\right)_{i, j, k+1 / 2}^{n} \approx \frac{u_{i, j, k+1}^{n}-u_{i, j, k}^{n}}{\delta z}
$$


Os valores de $u_{i, j, k+1}^{n}$ e $u_{i, j, k}^{n}$ são aproximados por valores de pontos próximos, em que esta componente da velocidade é definida pela discretização:

$$
u_{i, j, k+1}^{n}=\frac{u_{i+1 / 2, j, k+1}^{n}+u_{i-1 / 2, j, k+1}^{n}}{2}, \quad u_{i, j, k}^{n}=\frac{u_{i+1 / 2, j, k}^{n}+u_{i-1 / 2, j, k}^{n}}{2} .
$$

Porém, $u_{i, j, k+1 / 2}^{n}=0$, pela condição de contorno (ver equação (1.33)). Para que isto ocorra, considera-se na célula B que

$$
u_{i, j, k}^{n}=-u_{i, j, k+1}^{n} .
$$

Substituindo-se (3.85) e (3.84) em (3.83), obtém-se

$$
\left(\frac{\partial u}{\partial z}\right)_{i, j, k+1 / 2}^{n} \approx \frac{u_{i+1 / 2, j, k+1}^{n}+u_{i-1 / 2, j, k+1}^{n}}{\delta z}
$$

A mesma técnica é aplicada para $\left(\frac{\partial u}{\partial z}\right)_{i, j, k+1 / 2}^{n+1}$, e para as demais derivadas que aparecem nos sistemas de equações acima citados.

\subsection{Aproximação do tensor $\mathrm{S}$ em entradas e saídas de fluido}

As equações apresentadas na Seção 1.3 são aproximadas percorrendo-se as células inflow (I) e outflow (O) e atualizando-se as componentes de $\mathbf{S}$ como segue:

Célula inflow (I) em contato com célula $\mathbf{F}$ ou célula $\mathbf{S}$

Considerando-se entradas de fluido paralelas ao plano $y z$, existem dois casos:

Caso 1: célula I com face $(i+1 / 2)$ em contato com célula $\mathrm{F}$ ou $\mathrm{S}$ (fig. $3.12(\mathrm{a}))$. Nesse caso, quando se tratar de inflow linear, os valores das 


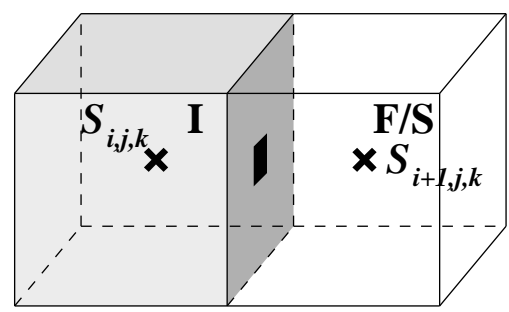

(a)

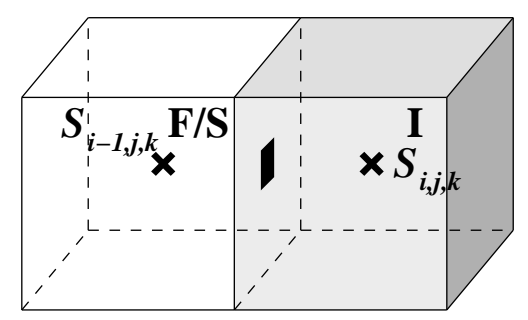

(b)

Figura 3.12: Configurações de células I com a face $(i+1 / 2)$ (a) ou $(i-1 / 2)$ em contato com célula F ou S.

componentes são dados por

$$
\begin{array}{lll}
S_{i, j, k}^{x x}=-S_{i+1, j, k}^{x x}, & S_{i, j, k}^{x y}=-S_{i+1, j, k}^{x y}, & S_{i, j, k}^{x z}=-S_{i+1, j, k}^{x z}, \\
S_{i, j, k}^{y y}=-S_{i+1, j, k}^{y y}, & S_{i, j, k}^{y z}=-S_{i+1, j, k}^{y z}, & S_{i, j, k}^{z z}=-S_{i+1, j, k}^{z z} .
\end{array}
$$

Caso 2: célula I com face $(i-1 / 2)$ em contato com célula $\mathrm{F}$ ou $\mathrm{S}$ (fig. 3.12(b)). Nesse caso, os valores das componentes são dados por

$$
\begin{array}{lll}
S_{i, j, k}^{x x}=-S_{i-1, j, k}^{x x}, & S_{i, j, k}^{x y}=-S_{i-1, j, k}^{x y}, & S_{i, j, k}^{x z}=-S_{i-1, j, k}^{x z}, \\
S_{i, j, k}^{y y}=-S_{i-1, j, k}^{y y}, & S_{i, j, k}^{y z}=-S_{i-1, j, k}^{y z}, & S_{i, j, k}^{z z}=-S_{i-1, j, k}^{z z} .
\end{array}
$$

No caso de entradas de fluido paralelos aos outros planos, o procedimento é análogo.

Célula outflow $(\mathrm{O})$ em contato com célula $\mathbf{F}$ ou célula $\mathrm{S}$

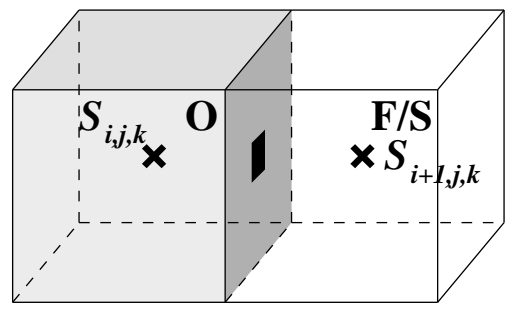

(a)

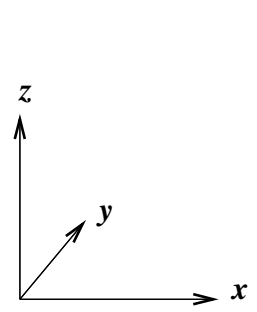

$x$

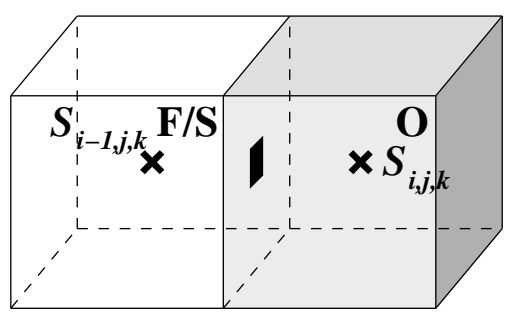

(b)

Figura 3.13: Configurações de células $\mathrm{O}$ com a face $(i+1 / 2)$ (a) ou $(i-1 / 2)$ (b) em contato com célula F ou S.

Considerando-se saídas de fluido paralelas ao plano yz, existem dois casos: 
Caso 1: célula $\mathrm{O}$ com face $(i+1 / 2)$ em contato com célula $\mathrm{F}$ ou $\mathrm{S}$ (ver figura $3.13(\mathrm{a}))$.

Nesse caso, os valores das componentes são dados por

$$
\begin{array}{lll}
S_{i, j, k}^{x x}=S_{i+1, j, k}^{x x}, & S_{i, j, k}^{x y}=S_{i+1, j, k}^{x y}, & S_{i, j, k}^{x z}=S_{i+1, j, k}^{x z}, \\
S_{i, j, k}^{y y}=S_{i+1, j, k}^{y y}, & S_{i, j, k}^{y z}=S_{i+1, j, k}^{y z}, & S_{i, j, k}^{z z}=S_{i+1, j, k}^{z z} .
\end{array}
$$

Caso 2: célula $\mathrm{O}$ com face $(i-1 / 2)$ em contato com célula $\mathrm{F}$ ou $\mathrm{S}$ (ver figura 3.13(b)).

Nesse caso,

$$
\begin{array}{lll}
S_{i, j, k}^{x x}=S_{i-1, j, k}^{x x}, & S_{i, j, k}^{x y}=S_{i-1, j, k}^{x y}, & S_{i, j, k}^{x z}=S_{i-1, j, k}^{x z}, \\
S_{i, j, k}^{y y}=S_{i-1, j, k}^{y y}, & S_{i, j, k}^{y z}=S_{i-1, j, k}^{y z}, & S_{i, j, k}^{z z}=S_{i-1, j, k}^{z z} .
\end{array}
$$

No caso de saídas de fluido paralelos aos outros planos, o procedimento é análogo.

\subsection{Aproximação do tensor taxa de defor- mação D}

O cálculo do tensor não-newtoniano $\mathbf{S}$ por meio da equação constitutiva discreta (3.20) (ver também equação (3.5)) utiliza os valores do tensor taxa de deformação $\mathbf{D}$ nas células $\mathrm{F}$ e $\mathrm{S}$, e a aproximação dos termos convectivos $\nabla \cdot(\mathbf{u D})$ pelo método de alta ordem CUBISTA [38] requer os valores deste nas células do contorno da malha. Tais valores são obtidos como segue:

Em coordenadas cartesianas tridimensionais, o tensor taxa de deformação 
D é dado por

$$
\begin{aligned}
\mathbf{D} & =\left(\begin{array}{ccc}
D^{x x} & D^{x y} & D^{x z} \\
D^{x y} & D^{y y} & D^{y z} \\
D^{x z} & D^{y z} & D^{z z}
\end{array}\right) \\
& =\left(\begin{array}{ccc}
\frac{\partial u}{\partial x} & \frac{1}{2}\left[\frac{\partial u}{\partial y}+\frac{\partial v}{\partial x}\right] & \frac{1}{2}\left[\frac{\partial u}{\partial z}+\frac{\partial w}{\partial x}\right] \\
\frac{1}{2}\left[\frac{\partial u}{\partial y}+\frac{\partial v}{\partial x}\right] & \frac{\partial v}{\partial y} & \frac{1}{2}\left[\frac{\partial v}{\partial z}+\frac{\partial w}{\partial y}\right] \\
\frac{1}{2}\left[\frac{\partial u}{\partial z}+\frac{\partial w}{\partial x}\right] & \frac{1}{2}\left[\frac{\partial v}{\partial z}+\frac{\partial w}{\partial y}\right] & \frac{\partial w}{\partial z}
\end{array}\right) .
\end{aligned}
$$

\subsubsection{Aproximação de D nas células F e S}

Para as células cheias (F) e de superfície (S), temos que:

$$
\begin{gathered}
\left(D^{x x}\right)_{i, j, k}=\left(\frac{\partial u}{\partial x}\right)_{i, j, k}, \\
\left(D^{y y}\right)_{i, j, k}=\left(\frac{\partial v}{\partial y}\right)_{i, j, k}, \\
\left(D^{z z}\right)_{i, j, k}=\left(\frac{\partial w}{\partial z}\right)_{i, j, k}, \\
\left(D^{x y}\right)_{i, j, k}=\frac{1}{2}\left[\left(\frac{\partial u}{\partial y}\right)_{i, j, k}+\left(\frac{\partial v}{\partial x}\right)_{i, j, k}\right] \\
\left(D^{x z}\right)_{i, j, k}=\frac{1}{2}\left[\left(\frac{\partial u}{\partial z}\right)_{i, j, k}+\left(\frac{\partial w}{\partial x}\right)_{i, j, k}\right], \\
\left(D^{y z}\right)_{i, j, k}=\frac{1}{2}\left[\left(\frac{\partial v}{\partial z}\right)_{i, j, k}+\left(\frac{\partial w}{\partial y}\right)_{i, j, k}\right],
\end{gathered}
$$

onde

$$
\begin{aligned}
& \left(\frac{\partial u}{\partial x}\right)_{i, j, k}=\frac{u_{i+1 / 2, j, k}-u_{i-1 / 2, j, k}}{\delta x}, \\
& \left(\frac{\partial u}{\partial y}\right)_{i, j, k}=\frac{u_{i, j+1 / 2, k}-u_{i, j-1 / 2, k}}{\delta y} \\
& \left(\frac{\partial u}{\partial z}\right)_{i, j, k}=\frac{u_{i, j, k+1 / 2}-u_{i, j, k-1 / 2}}{\delta z}
\end{aligned}
$$




$$
\begin{aligned}
& \left(\frac{\partial v}{\partial x}\right)_{i, j, k}=\frac{v_{i+1 / 2, j, k}-v_{i-1 / 2, j, k}}{\delta x}, \\
& \left(\frac{\partial v}{\partial y}\right)_{i, j, k}=\frac{v_{i, j+1 / 2, k}-v_{i, j-1 / 2, k}}{\delta y}, \\
& \left(\frac{\partial v}{\partial z}\right)_{i, j, k}=\frac{v_{i, j, k+1 / 2}-v_{i, j, k-1 / 2}}{\delta z}, \\
& \left(\frac{\partial w}{\partial x}\right)_{i, j, k}=\frac{w_{i+1 / 2, j, k}-w_{i-1 / 2, j, k}}{\delta x}, \\
& \left(\frac{\partial w}{\partial y}\right)_{i, j, k}=\frac{w_{i, j+1 / 2, k}-w_{i, j-1 / 2, k}}{\delta y}, \\
& \left(\frac{\partial w}{\partial z}\right)_{i, j, k}=\frac{w_{i, j, k+1 / 2}-w_{i, j, k-1 / 2}}{\delta z} .
\end{aligned}
$$

Note que as derivadas das equações (3.94), (3.98) e (3.102) são obtidas diretamente. Entretanto, para as demais derivadas (derivadas cruzadas), são necessários valores de componentes da velocidade em pontos em que estas não estão definidas pela discretização. Neste caso, procede-se da mesma forma como explicado na seção 3.6 para a aproximação, por exemplo, da equação $(3.95)$.

\subsubsection{Aproximação de D nas células de contorno}

\section{Contornos paralelos ao plano $x y$}

Temos neste caso (veja também seção 1.4.1) que

$$
\left(D^{x z}\right)_{i, j, k}=\frac{1}{2}\left(\frac{\partial u}{\partial z}\right)_{i, j, k}, \quad\left(D^{y z}\right)_{i, j, k}=\frac{1}{2}\left(\frac{\partial v}{\partial z}\right)_{i, j, k}
$$

e as demais componentes de $\mathbf{D}$ são todas nulas. 
Contornos paralelos ao plano $x z$

As únicas componentes de $\mathbf{D}$ não nulas (veja também seção 1.4.2) são

$$
\left(D^{x y}\right)_{i, j, k}=\frac{1}{2}\left(\frac{\partial u}{\partial y}\right)_{i, j, k}, \quad\left(D^{y z}\right)_{i, j, k}=\frac{1}{2}\left(\frac{\partial w}{\partial y}\right)_{i, j, k}
$$

\section{Contornos paralelos ao plano $y z$}

Por fim, nesta situação as únicas componentes de $\mathbf{D}$ não nulas (veja também seção 1.4.3) são

$$
\left(D^{x y}\right)_{i, j, k}=\frac{1}{2}\left(\frac{\partial v}{\partial x}\right)_{i, j, k}, \quad\left(D^{x z}\right)_{i, j, k}=\frac{1}{2}\left(\frac{\partial w}{\partial x}\right)_{i, j, k}
$$

As derivadas nas equações (3.103)-(3.105) são calculadas conforme ilustrado na seção 3.9.5 para $\left(\frac{\partial u}{\partial z}\right)_{i, j, k}$.

\subsubsection{Aproximação de D em entradas e saídas de fluido}

No caso de entradas de fluido lineares e saídas de fluido, procede-se da mesma maneira como feito para o tensor $\mathbf{S}$ - veja seção 3.10. 


\section{Capítulo 4}

\section{Resultados de validação}

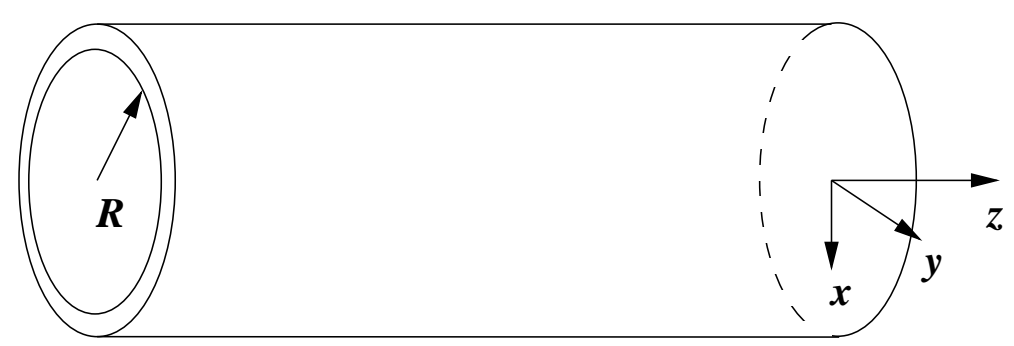

Figura 4.1: Solução numérica do escoamento de um fluido de Maxwell num tubo: descrição do domínio computacional.

As equações de diferenças finitas obtidas no Capítulo 3 foram implementadas no sistema de simulação Freeflow3D [7] com o objetivo de simular escoamentos tridimensionais governados pela equação constitutiva de Maxwell.

Para validar o método numérico apresentado nesse trabalho vamos utilizar o escoamento desenvolvido em um tubo e comparar as soluções numéricas com as respectivas soluções analíticas como segue.

Considere o escoamento em um tubo como mostrado na figura 4.1. Para escoamentos desenvolvidos temos que $\mathbf{u}=(0,0, w(x, y))$, a pressão varia linearmente com $z$ e as componentes do tensor não-newtoniano $\mathbf{S}$ variam somente com $x$ e $y$, ou seja,

$$
p=p(z)=\text { constante e } \frac{\partial}{\partial t}=\frac{\partial}{\partial z} \equiv 0 .
$$


Nesse caso, a equação da continuidade é satisfeita e as equações (1.22)-(1.30) se reduzem a

$$
\begin{gathered}
\frac{\partial S^{x x}}{\partial x}+\frac{\partial S^{x y}}{\partial y}=0 \\
\frac{\partial S^{x y}}{\partial x}+\frac{\partial S^{y y}}{\partial y}=0 \\
-\frac{\partial p}{\partial z}+\frac{1}{R e}\left(\frac{\partial^{2} w}{\partial x^{2}}+\frac{\partial^{2} w}{\partial y^{2}}\right)+\frac{\partial S^{x z}}{\partial x}+\frac{\partial S^{y z}}{\partial y}=0 \\
S^{x x}=0 \\
S^{y y}=0 \\
S^{z z}-2 W e\left(\frac{\partial w}{\partial x} S^{x z}+\frac{\partial w}{\partial y} S^{y z}\right)=2 \frac{W e}{R e}\left[\left(\frac{\partial w}{\partial x}\right)^{2}+\left(\frac{\partial w}{\partial y}\right)^{2}\right] \\
S^{x y}=0 \\
S^{x z}-W e\left(\frac{\partial w}{\partial x} S^{x x}+\frac{\partial w}{\partial y} S^{x y}\right)=0 \\
S^{y z}-W e\left(\frac{\partial w}{\partial x} S^{x y}+\frac{\partial w}{\partial y} S^{y y}\right)=0 .
\end{gathered}
$$

As equações (4.2)-(4.10) formam um sistema não-linear com 9 equações para 8 incógnitas $\left(w, p, S^{x x}, S^{y y}, S^{z z}, S^{x y}, S^{x z}, S^{y z}\right)$ que pode ser facilmente resolvido como segue.

Das equações (4.5), (4.6) e (4.8) vemos que $S^{x x}=S^{y y}=S^{x y}=0$, e das equações (4.9) e (4.10) obtém-se $S^{x z}=S^{y z}=0$. Nesse caso, as equações (4.2) e (4.3) são satisfeitas e restam apenas as equações (4.4) e (4.7), que formam um sistema com 2 equações para 3 incógnitas $\left(w(x, y), \frac{\partial p}{\partial z}, S^{z z}\right)$ dado por

$$
\begin{gathered}
-\frac{\partial p}{\partial z}+\frac{1}{R e}\left(\frac{\partial^{2} w}{\partial x^{2}}+\frac{\partial^{2} w}{\partial y^{2}}\right)=0, \\
S^{z z}=2 \frac{W e}{R e}\left[\left(\frac{\partial w}{\partial x}\right)^{2}+\left(\frac{\partial w}{\partial y}\right)^{2}\right] .
\end{gathered}
$$

Para obter uma solução desse sistema, assume-se que a velocidade $w(x, y)$ é conhecida e resolve-se (4.11) e (4.12) para $\frac{\partial p}{\partial z}$ e $S^{z z}$. De fato, se na entrada 
do canal impusermos escoamento de Poiseuille dado por

$$
w(x, y)=2 U\left[R^{2}-\left(x^{2}+y^{2}\right)\right] / R^{2},
$$

então, de (4.11) e (4.12) obtemos:

$$
\begin{gathered}
\frac{\partial p}{\partial z}=-\frac{8}{R e} \frac{U}{R^{2}}=k<0, \\
S^{z z}=32 \frac{W e}{R e} \frac{U^{2}}{R^{4}}\left(x^{2}+y^{2}\right) .
\end{gathered}
$$

Logo, no estado estacionário do escoamento em um tubo utilizando a equação constitutiva de Maxwell, apenas a componente $S^{z z}$ é não-nula, e o campo de pressões descresce linearmente na direção $z$ positiva.

Para validarmos o método numérico desenvolvido nesse trabalho simulamos o escoamento totalmente desenvolvido em um tubo e comparamos as soluções numéricas com as soluções analíticas dadas pelas equações (4.13) e (4.15).

Utilizamos $R=1 \mathrm{~m}, U=1 \mathrm{~m} / \mathrm{s}, \nu=2 \mathrm{~m}^{2} / \mathrm{s}, \lambda=0.4 \mathrm{~s}$ e desprezamos a gravidade. Logo, temos $R e=U R / \nu=0.5$ e $W e=\lambda U / L=0.4$. Para analisar a convergência do método numérico, utilizamos três malhas:

- Malha M1 - grossa: $\delta x=\delta y=\delta z=0.16666667(12 \times 12 \times 60$ células computacionais);

- Malha M2 - intermediária: $\delta x=\delta y=\delta z=0.125(16 \times 16 \times 80$ células computacionais);

- Malha M3 - fina: $\delta x=\delta y=\delta z=0.1(20 \times 20 \times 100$ células computacionais).

Na malha M1 a seção transversal do tubo possui 112 células computacionais ao passo que na malha M2 são 208 células e na malha M3 são 316 células computacionais. 


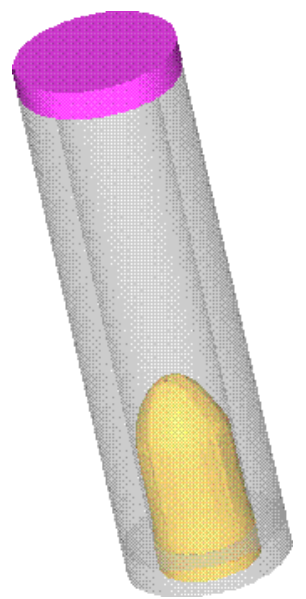

(a) $t=2.5 \mathrm{~s}$

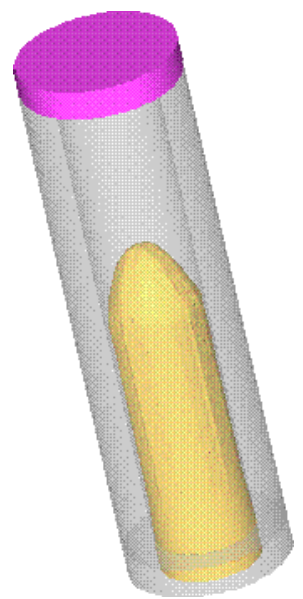

(b) $t=5 \mathrm{~s}$

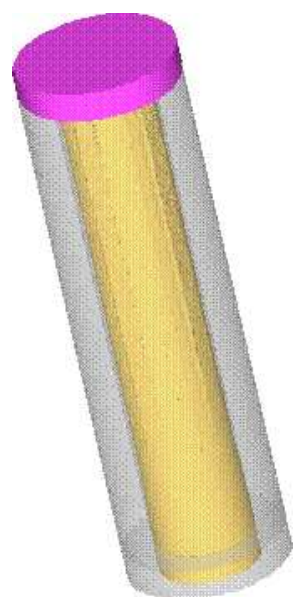

(c) $t=20 \mathrm{~s}$

Figura 4.2: Evolução do enchimento do tubo.

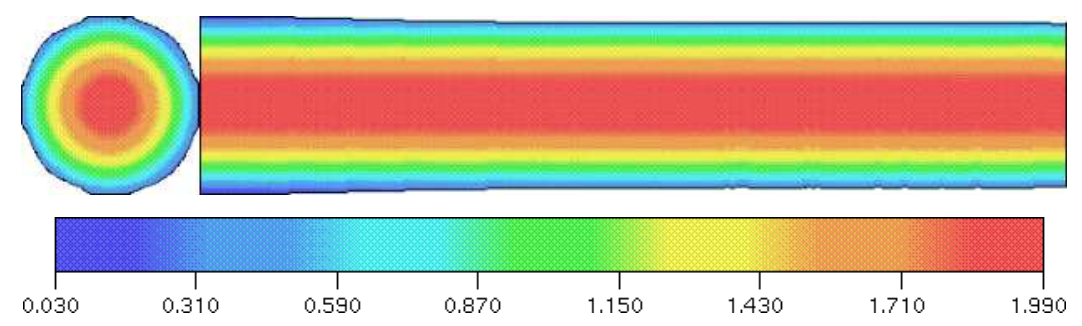

Figura 4.3: Visualização de $w$ na saída de fluido e ao longo do tubo em $t=20 s$.

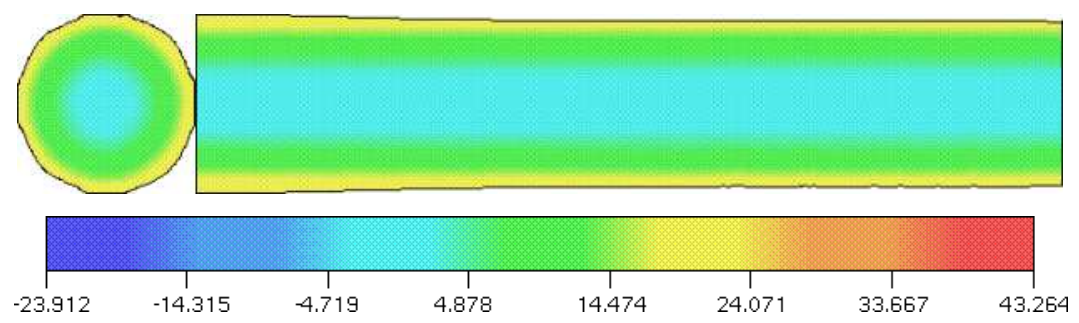

Figura 4.4: Visualização de $S^{z z}$ na saída de fluido e ao longo do tubo em $t=20 s$. 
A simulação com o código Freeflow3D iniciou com o tubo vazio e o fluido foi injetado na entrada do tubo até que o estado estacionário fosse atingido.

As figuras 4.2(a) e 4.2(b) ilustram algumas etapas da simulação onde podemos observar que o tubo está em fase de enchimento. A figura 4.2(c) mostra a configuração do escoamento no tempo $t=20$ s onde vemos que o tubo encontra-se totalmente cheio, e as figuras 4.3 e 4.4 mostram que as isolinhas estão todas paralelas, o que indica que o estado estacionário foi atingido. Para verificarmos a convergência do método desenvolvido nesse trabalho, comparamos as soluções numéricas obtidas na seção transversal do tubo adjacente à saída de fluido nas malhas M1, M2 e M3 com a solução analítica dada pelas equações (4.13) e (4.12).

As figuras 4.6(a) e 4.6(b) mostram as soluções obtidas utilizando as três malhas e a solução analítica, onde nota-se que as soluções numéricas se aproximam da solução analítica à medida que a malha é refinada. Para quantificarmos esse fato, calculamos o erro relativo entre a solução analítica e a solução numérica, definido por

$$
E(\text { Sol Num })=\frac{\sum_{i, j}(\text { Sol Ex }- \text { Sol Num })^{2}}{\sum_{i, j}(\text { Sol Ex })^{2}} .
$$

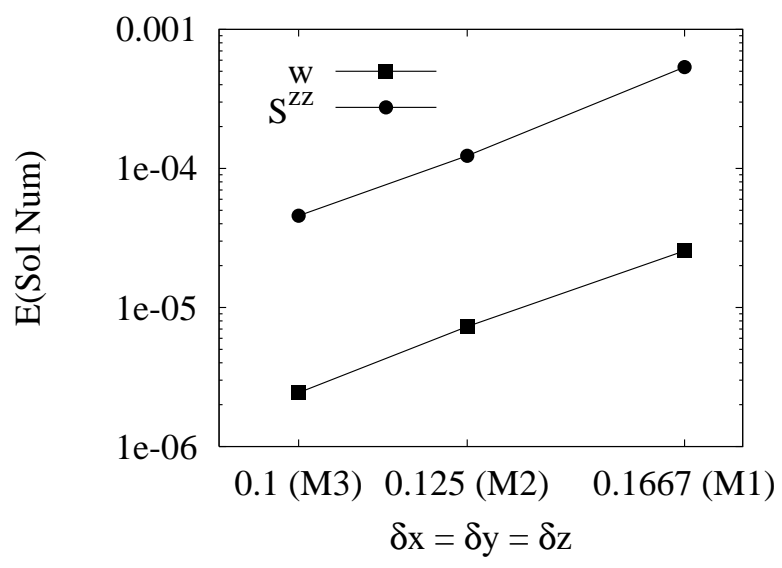

Figura 4.5: Decaimento do erro relativo com o refinamento da malha.

A tabela 4.1 e a figura 4.5 mostram o erro cometido entre a solução numérica e a solução analítica utilizando as três malhas. Pode-se observar na 


\begin{tabular}{|c|c|c|c|}
\hline Malha & M1 & M2 & M3 \\
\hline$E(w(x, y))$ & $2.553 \times 10^{-5}$ & $7.289 \times 10^{-6}$ & $2.437 \times 10^{-6}$ \\
\hline$E\left(S^{z z}(x, y)\right)$ & $5.353 \times 10^{-4}$ & $1.232 \times 10^{-4}$ & $4.573 \times 10^{-5}$ \\
\hline
\end{tabular}

Tabela 4.1: Erro relativo na seção transversal adjacente à saída de fluido.

tabela 4.1 que o erro decresce à medida que a malha computacional é refinada, demonstrando assim a convergência do método numérico desenvolvido.

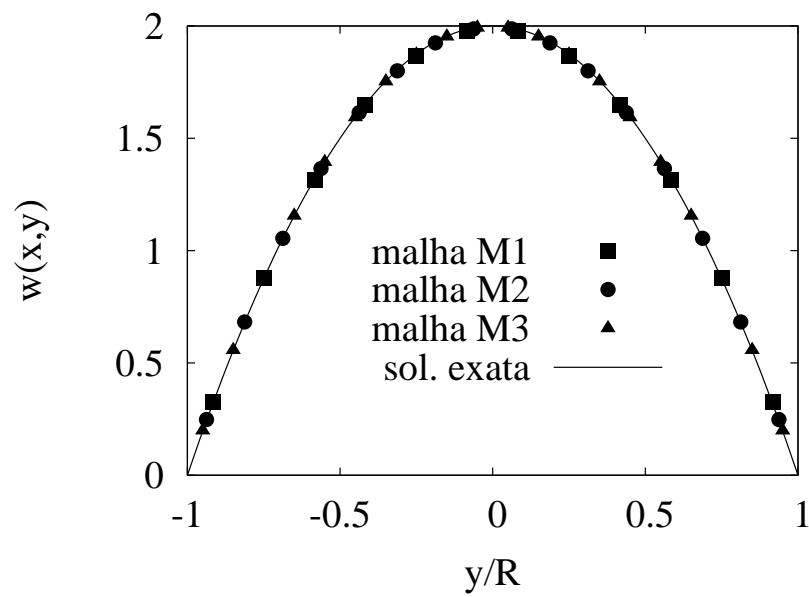

(a)

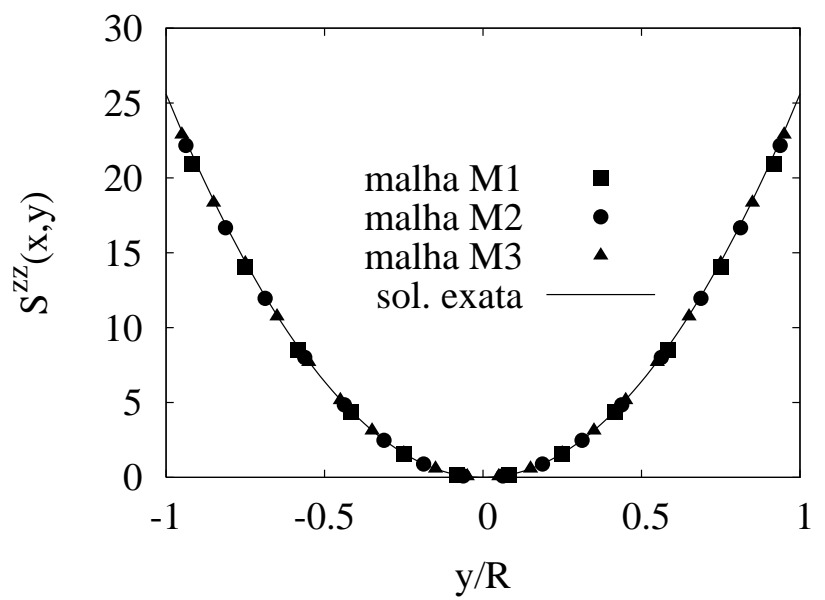

(b)

Figura 4.6: Comparação entre a solução analítica e as soluções numéricas na saída do fluido, para $x=0$. 


\section{Capítulo 5}

\section{Simulação numérica de escoamentos viscoelásticos com superfícies livres}

\subsection{Simulação numérica do jato oscilante}

Nesta seção apresentamos resultados numéricos obtidos na simulação do escoamento tridimensional produzido por um jato incidindo sobre uma placa plana. Sabe-se que sob certas condições, pode ocorrer o fenômeno conhecido como jato oscilante (também conhecido como flambagem). Esse problema tem aplicações industriais, sendo que vários pesquisadores têm estudado o jato oscilante experimentalmente (por ex. [41-44]) como também numericamente [45, 46]. Em particular, Cruickshank e Munson [44] realizaram uma série de experimentos e concluíram que para um jato newtoniano tridimensional, o fenômeno do jato oscilante ocorre se as condições abaixo são satisfeitas:

$$
\begin{gathered}
R e=\rho U D / \mu<1.2 \\
H / D>7.2
\end{gathered}
$$

Na condição (5.1b), $H$ é a distância do injetor de fluido à placa plana e $D$ é o diâmetro do injetor. Duas simulações foram realizadas utilizando-se o mo- 


\begin{tabular}{|c|c|c|}
\hline parâmetro & Problema 1 & Problema 2 \\
\hline$R e$ & 0.5 & 1.3 \\
$U(\mathrm{~m} / \mathrm{s})$ & 1 & 0.5 \\
$\mu(\mathrm{Pa} \cdot \mathrm{s})$ & 12 & $\approx 2.3077$ \\
$\lambda(\mathrm{s})-$ apenas UCM & 0.003 & 0.006 \\
\hline$H(\mathrm{~m})$ & \multicolumn{2}{|c|}{0.12} \\
$D(\mathrm{~m})$ & \multicolumn{2}{|c|}{0.006} \\
$\rho\left(\mathrm{kg} / \mathrm{m}^{3}\right)$ & \multicolumn{2}{|c|}{1000} \\
$W e-$ apenas UCM & \multicolumn{2}{|c|}{0.5} \\
\hline
\end{tabular}

Tabela 5.1: Dados de entrada para as simulações numéricas do problema do jato oscilante.

delo de Maxwell: uma simulação com $R e=0.5$ (denominado Problema 1) e outra simulação empregando $R e=1.3$ (denominado Problema 2). O respectivo caso newtoniano também foi simulado. A Tabela 5.1 fornece os dados de entrada utilizados nas simulações realizadas. Observamos que $H / D=20$ de forma que a condição (5.1b) é satisfeita em ambas as simulações, enquanto que a condição (5.1a) somente é satisfeita para o Problema 1.

Utilizamos uma placa rígida com dimensões $70 \mathrm{~mm} \times 70 \mathrm{~mm} \times 3 \mathrm{~mm}$ e uma malha com espaçamento $\delta x=\delta y=\delta z=1 \mathrm{~mm}$. O domínio computacional utilizado teve dimensões $70 \mathrm{~mm} \times 70 \mathrm{~mm} \times 120 \mathrm{~mm}$ produzindo uma malha com $70 \times 70 \times 120$ células (ver Figura 5.1).

O código Freeflow3D utilizou esses dados de entrada e simulou o Problema 1 e o Problema 2 até o instante $t=1.25 \mathrm{~s}$. Os resultados obtidos nessas simulações estão ilustrados nas figuras 5.5 a 5.8, onde podemos observar a evolução temporal do escoamento. Com relação ao jato newtoniano, os resultados obtidos são compatíveis com a teoria de Cruickshank e Munson [44]. No Problema 1 o jato newtoniano satisfaz ambas as condições (5.1a) e (5.1b) e portanto apresentou o efeito do jato oscilante enquanto que no Problema 2 a condição (5.1a) não foi satisfeita e portanto os resultados mostram que o jato newtoniano não apresentou esse efeito.

Os resultados obtidos com o fluido de Maxwell mostram que houve o efeito do jato oscilante para ambos os valores do número de Reynolds, sendo que para $R e=0.5$ (Problema $\mathbf{1}$ ) este efeito foi mais acentuado quando 

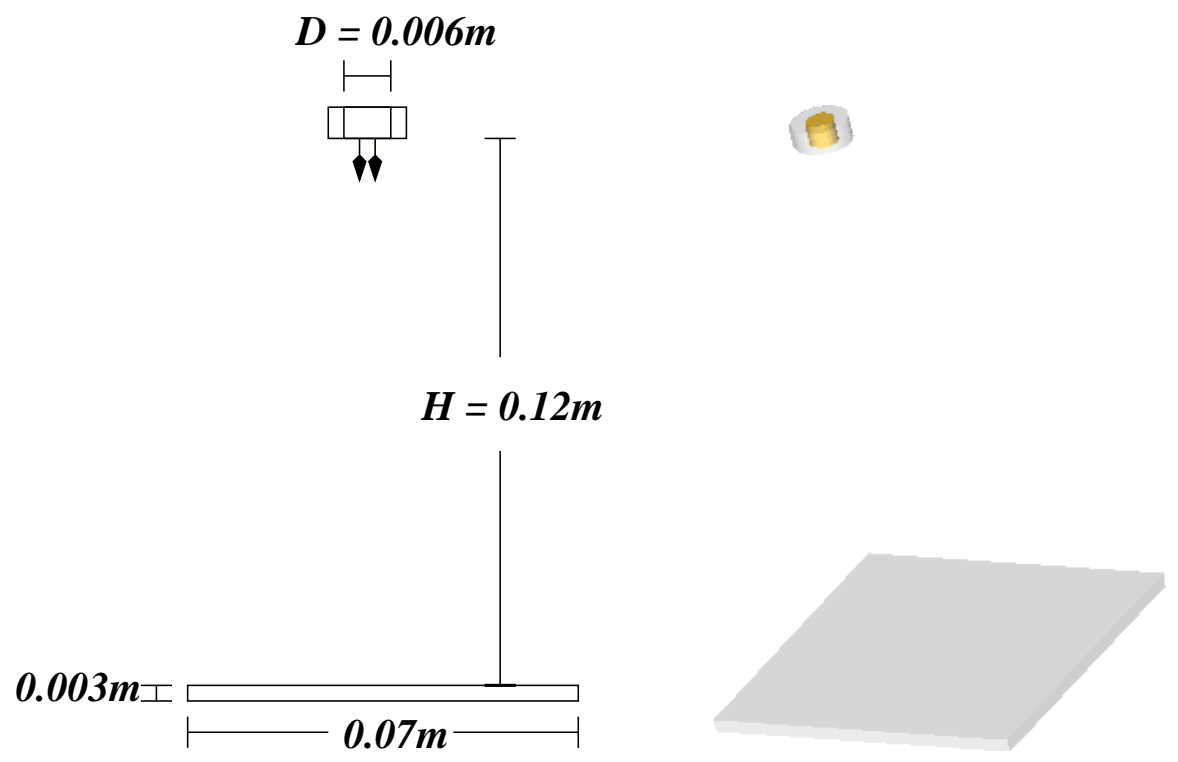

Figura 5.1: Domínio computacional utilizado na simulação numérica do jato oscilante.

comparado com o jato newtoniano. A figura 5.5 mostra que antes dos jatos atingirem a placa plana os escoamentos são semelhantes. A diferença entre os dois escoamentos começa após os jatos atingirem a placa plana em $t=0.125 \mathrm{~s}$. O jato newtoniano tem inicialmente o seu diâmetro aumentado próximo a placa enquanto que o jato com o fluido de Maxwell já apresenta o efeito do jato oscilante conhecido como coiling em $t=0.25 \mathrm{~s}$. De fato, esses dois efeitos foram observados no trabalho experimental realizado por Cruickshank e Munson [44]. A partir de $t=0.375 \mathrm{~s}$, o jato newtoniano começa a apresentar o efeito do jato oscilante e nos tempos subseqüentes o escoamento produzido pelo jato newtoniano torna-se semelhante ao escoamento do jato viscoelástico. No tempo $t=1.25 \mathrm{~s}$ podemos observar que a principal diferença entre os dois escoamentos está no efeito coiling apresentado pelos dois jatos: o jato com o fluido de Maxwell apresentou mais dobras, o que mostra que a freqüência do coiling foi maior que a freqüência do jato newtoniano. Os resultados obtidos nas simulações com $R e=1.3$ (Problema 2) até o instante $t=0.25 \mathrm{~s}$ (ver figura 5.7) não mostram grandes diferenças entre o jato newtoniano e o jato viscoelástico. Nesse problema o efeito da aceleração da gravidade é maior, o que causou uma diminuição do diâmetro dos jatos 
no momento que estes atingiram a placa rígida. Nesse caso, o aumento das forças inerciais juntamente com as forças gravitacionais fizeram com que o jato newtoniano escoasse radialmente sem apresentar os efeitos do jato oscilante, conforme previsto por Cruickshank e Munson [44]. No entanto, o jato viscoelático apresentou o efeito flambagem numa região logo acima da placa rígida. Acreditamos que esse efeito seja causado pelo aumento da viscosidade extensional nessa região. Esse fato foi demonstrado por Paulo et al. [33, 34] para problemas bidimensionais utilizando o modelo PTT.

\subsection{Simulação numérica do inchamento do ex- trudado}

Nesta seção são apresentados resultados numéricos das simulações do fenômeno do inchamento do extrudado. Esta situação geralmente ocorre quando o fluido é expelido para a atmosfera a partir de um tubo com diâmetro $D$, e corresponde ao aumento do diâmetro do jato após este deixar o tubo, que atinge um valor máximo $D_{\max }$. Sabe-se que quanto maior a elasticidade do fluido (representada pelo número de Weissenberg $W e$ ) maior é a taxa de inchamento $S_{r}=D_{\max } / D$. Esse fenômeno está presente em diversas aplicações industriais que envolvem a extrusão de polímeros e ligas metálicas e tem sido objeto de estudo de vários pesquisadores, como por exemplo [30, 47-55].

Para demonstrar que o método numérico apresentado nesse trabalho pode simular escoamentos de fluidos viscoelásticos, o código Freeflow3D foi aplicado para simular o inchamento do extrudado de um fluido modelado pela equação constitutiva de Maxwell. Consideramos um tubo circular com comprimento $L=5 \mathrm{~m}$ e raio $R=1 \mathrm{~m}$ conforme mostrado na figura 5.2. Na entrada do tubo - representada pela superfície amarela - foi imposto escoamento de Poiseuille com velocidade média $U=1 \mathrm{~m} / \mathrm{s}$ conforme especificado no Capítulo 4 por meio das equações (4.13) e (4.15). As simulações começaram com o tubo vazio e o fluido foi injetado até atingir a saída do tubo onde deverá ocorrer o fenômeno do inchamento do extrudado. Uma saída de 
fluido, representada por uma superfície de cor vermelha, foi colocada a uma distância $2 L$ da saída do tubo (apenas para limitar o tamanho do domínio computacional, não sendo relevante para a análise dos resultados obtidos). Para a viscosidade cinemática do fluido, foi assinalado o valor $\nu=1 \mathrm{~m}^{2} / \mathrm{s}^{2}$ de modo que $R e=U R / \nu=1$. Uma malha de $40 \times 40 \times 150$ células, com espaçamento $\delta x=\delta y=\delta z=0.1 \mathrm{~m}$ foi utilizada. Os efeitos gravitacionais são desprezados.
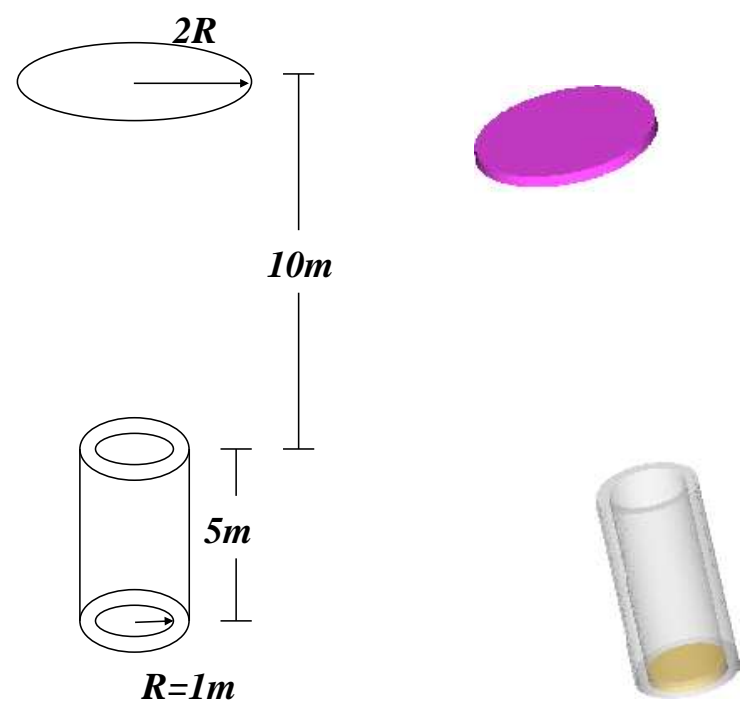

Figura 5.2: Domínio computacional utilizado na simulação numérica do inchamento do extrudado.

Foram realizadas cinco simulações: uma utilizando fluido newtoniano $(\mathrm{We}=0)$; e outras quatro para o fluido de Maxwell, onde o tempo de relaxação do fluido assumiu os valores $\lambda=0.1 \mathrm{~s}, 0.2 \mathrm{~s}, 0.3 \mathrm{~s}$ e $0.4 \mathrm{~s}$ e portanto, os números de Weissenberg $(W e=\lambda U / R)$ utilizados nessas simulações foram $W e=0.1,0.2,0.3$ e 0.4 . As figuras 5.9 e 5.10 mostram a evolução temporal do escoamento do jato obtida nas cinco simulações. No tempo $t=5 \mathrm{~s}$ o jato está começando a sair do tubo e os escoamentos ainda são semelhantes; porém à medida que o tempo avança os jatos são extrudados na atmosfera e já se observa a ocorrência do fenômeno do inchamento nas simulações $(t=7.5 \mathrm{~s})$. A partir do instante $t=10$ s nota-se a superfície livre do fluido newtoniano não sofreu alterações com relação ao instante anterior, apenas avançando de 
maneira a se afastar do tubo. Tal situação aparentemente também se observou para o jato viscoelástico em que $W e=0.1$. Para $W e=0.2,0.3 \mathrm{e}$ 0.4, nota-se que o inchamento ainda não ocorreu por completo. Porém, no tempo subseqüente, $t=15 \mathrm{~s}$, observa-se que a superfície livre de todos os jatos avançou, não ocorrendo variação na taxa de inchamento, o que permite portanto que esta possa ser estimada. Os valores de $S_{r}$ variaram de 1.108 a 1.350 para o fluido de Maxwell; esses resultados são coerentes com os resultados encontrados na literatura. À medida que o número de Weissenberg aumenta, maiores são os efeitos viscoelásticos, resultando em uma taxa de inchamento cada vez maior do fluido extrudado, e devido à conservação de massa, uma velocidade média $\bar{U}$ após a extrusão cada vez menor. O fluido newtoniano apresentou a menor taxa de inchamento, e portanto, a maior velocidade $\bar{U}$ entre as simulações realizadas, ao passo que o fluido viscoelástico com $W e=0.4$ apresentou respectivamente a maior taxa de inchamento e a menor velocidade após a extrusão.

A Tabela 5.2 apresenta os dados quantitativos obtidos nas cinco simulações realizadas.

\begin{tabular}{|c|c|c|c|c|c|}
\hline & newtoniano & $W e=0.1$ & $W e=0.2$ & $W e=0.3$ & $W e=0.4$ \\
\hline$S_{r}=D_{\max } / D$ & 1.083 & 1.108 & 1.167 & 1.3 & 1.350 \\
$\bar{U}(\mathrm{~m} / \mathrm{s})$ & 0.827 & 0.787 & 0.707 & 0.607 & 0.547 \\
\hline
\end{tabular}

Tabela 5.2: Resultados obtidos nas simulações do problema do inchamento do extrudado.

De acordo com Tanner [47], para escoamentos com simetria radial, uma estimativa para $S_{r}$ é dada pela equação

$$
S_{r}=0.13+\left[1+\frac{1}{2}\left(\frac{T^{z z}-T^{r r}}{2 T^{r z}}\right)^{2}\right]^{1 / 6}
$$

onde 1.13 é a taxa de inchamento para escoamentos de fluidos newtonianos $(W e=0)$ a baixos números de Reynolds, e $T^{z z}, T^{r r}$ e $T^{r z}$ são tensores extra-tensão dados em coordenadas cilíndricas. No caso do fluido de Maxwell apresentado neste projeto, e com base em resultado análogo obtido para escoamentos com simetria radial do fluido Oldroyd-B [32], podemos reescrever 


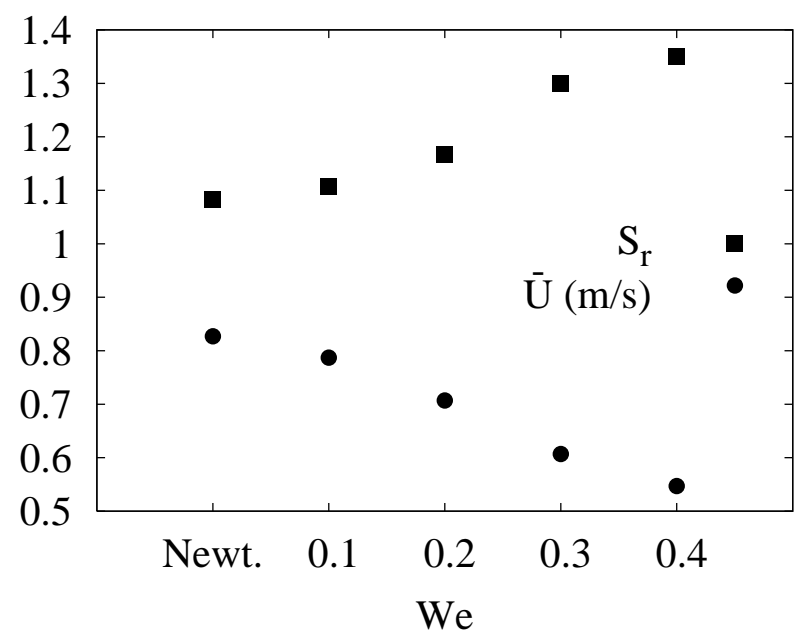

Figura 5.3: Resultados quantitativos das simulações do inchamento do extrudado.

(5.2) como

$$
S_{r}=0.13+\left[1+8 W e^{2}\right]^{1 / 6} .
$$

A Figura 5.4 compara a teoria de Tanner [47] e os resultados obtidos. Acreditamos que a diferença nos resultados deve-se não somente ao fato de que a teoria de Tanner se aplica a escoamentos com simetria radial e $R e \approx 0$ - no caso deste trabalho utilizamos o espaço cartesiano e $R e=1$ - mas também ao fato de que a equação (5.2) seja baseada em uma simplificação grosseira de um escoamento complexo na saída do tubo (veja [56]). Outros resultados numéricos da taxa de inchamento $S_{r}$ para $0 \leq W e \leq 0.5$ podem ser encontrados em [29].

Nas simulações realizadas não se observou o retardamento do inchamento do extrudado, que consiste num atraso do inchamento do fluido, que após deixar o tubo somente incha a uma certa distância deste. De acordo com o trabalho de Joseph e seus colaboradores [52-55], para que ocorra o retardamento do inchamento do extrudado, no caso do fluido de Maxwell, é preciso que o número de Mach $M a=\sqrt{R e W e}$ seja maior do que 1. Entretanto, para as simulações realizadas, temos $M a=0.316,0.447,0.548$ e 0.632 para $W e=0.1,0.2,0.3$ e 0.4 , respectivamente. 


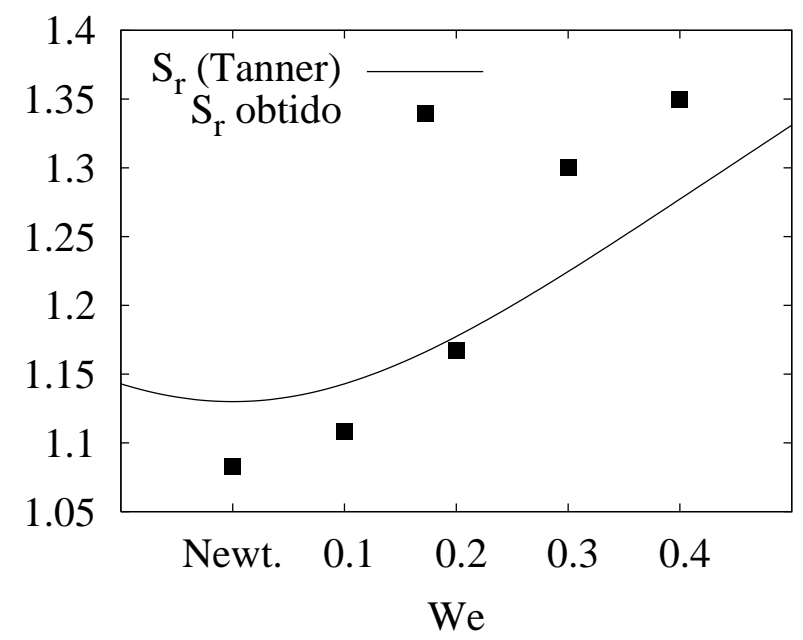

Figura 5.4: Taxa de inchamento $S_{r}$ : comparação entre os resultados obtidos e resultados teóricos de Tanner. 


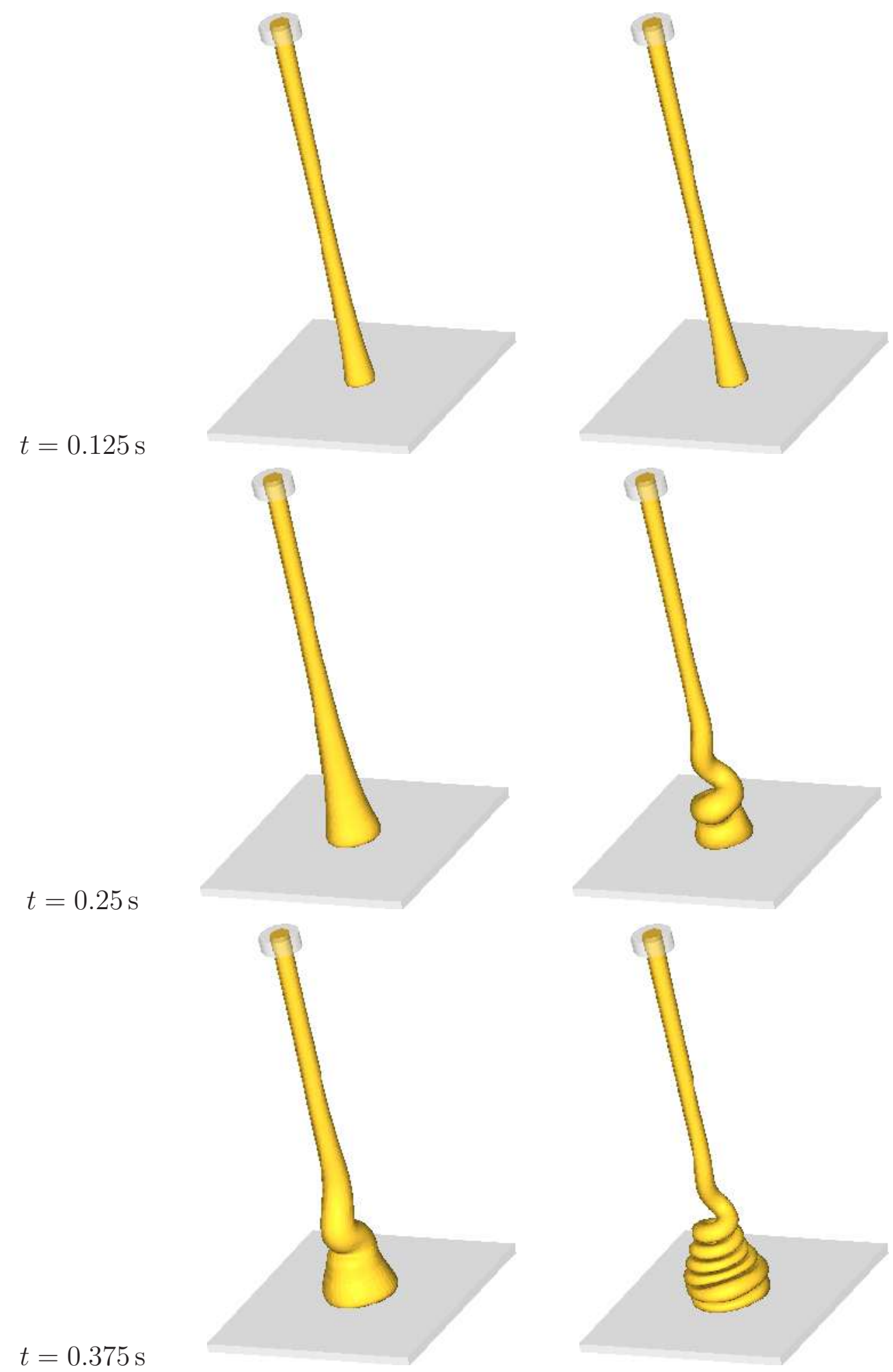

Figura 5.5: Simulação para $R e=0.5$ (Problema 1). À esquerda, fluido newtoniano; à direita, fluido de Maxwell. 


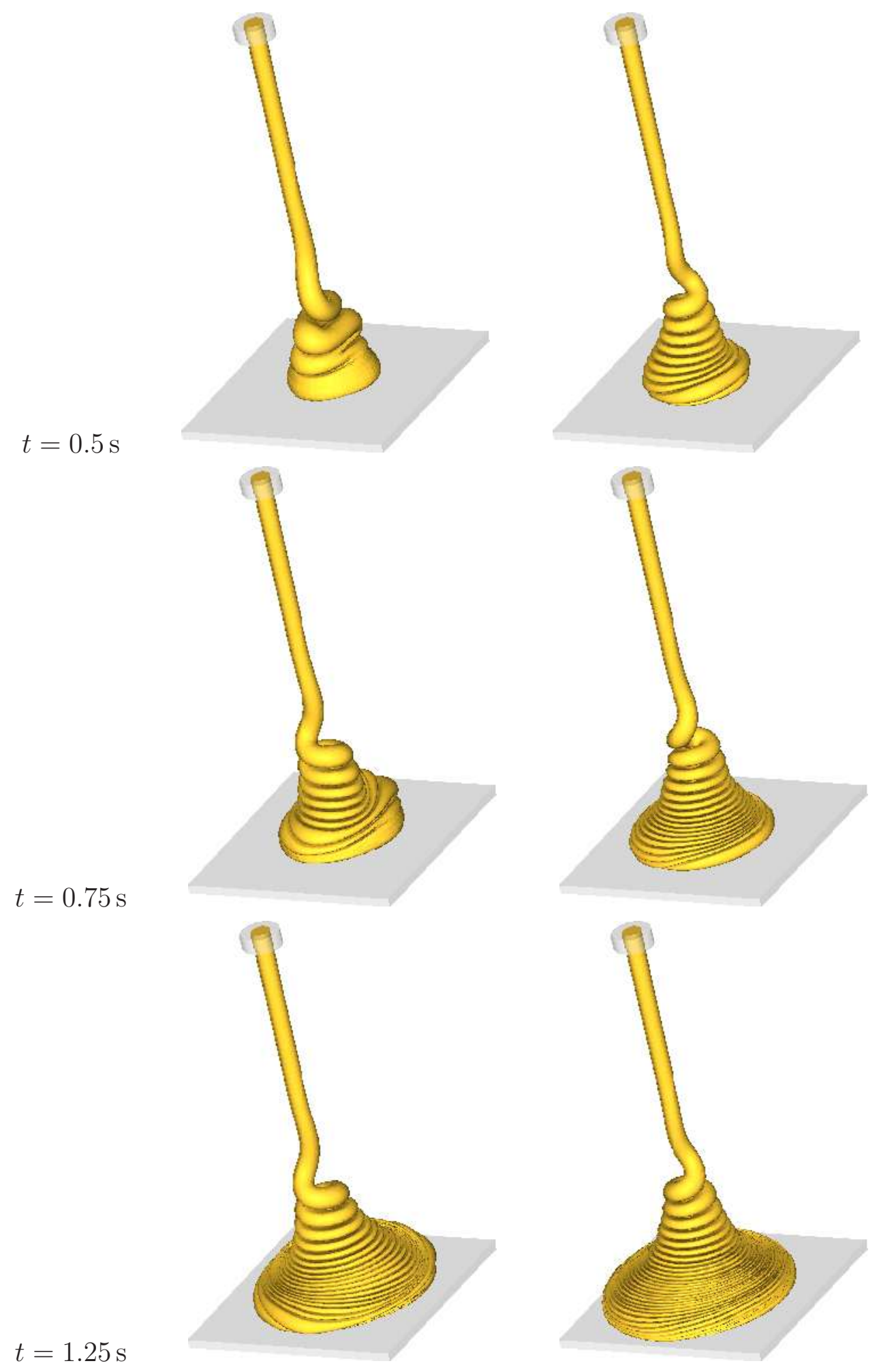

Figura 5.6: (cont.) Simulação para $R e=0.5$ (Problema 1). À esquerda, fluido newtoniano; à direita, fluido de Maxwell. 


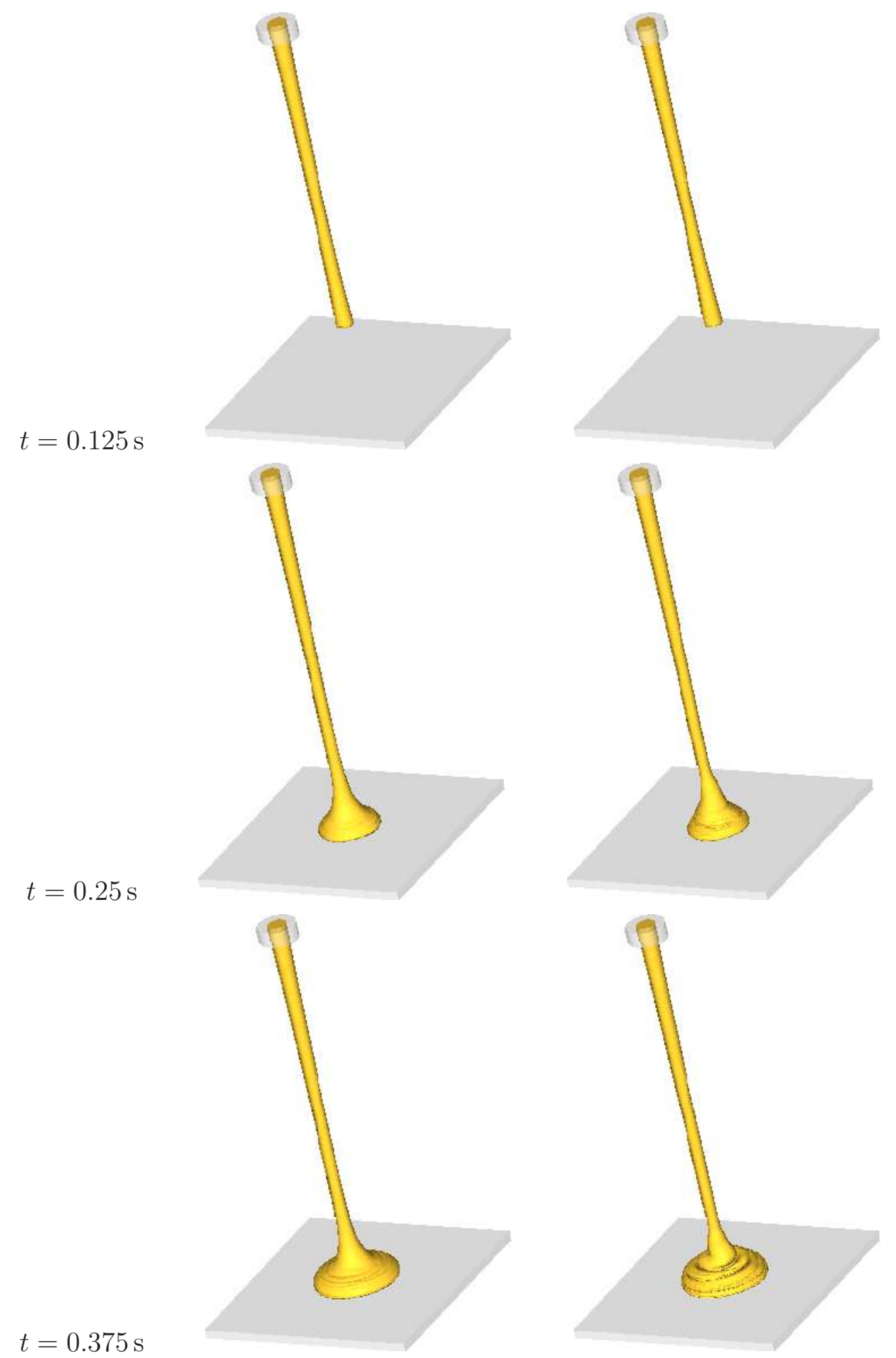

Figura 5.7: Simulação para $R e=1.3$ (Problema 2). À esquerda, fluido newtoniano; à direita, fluido de Maxwell. 


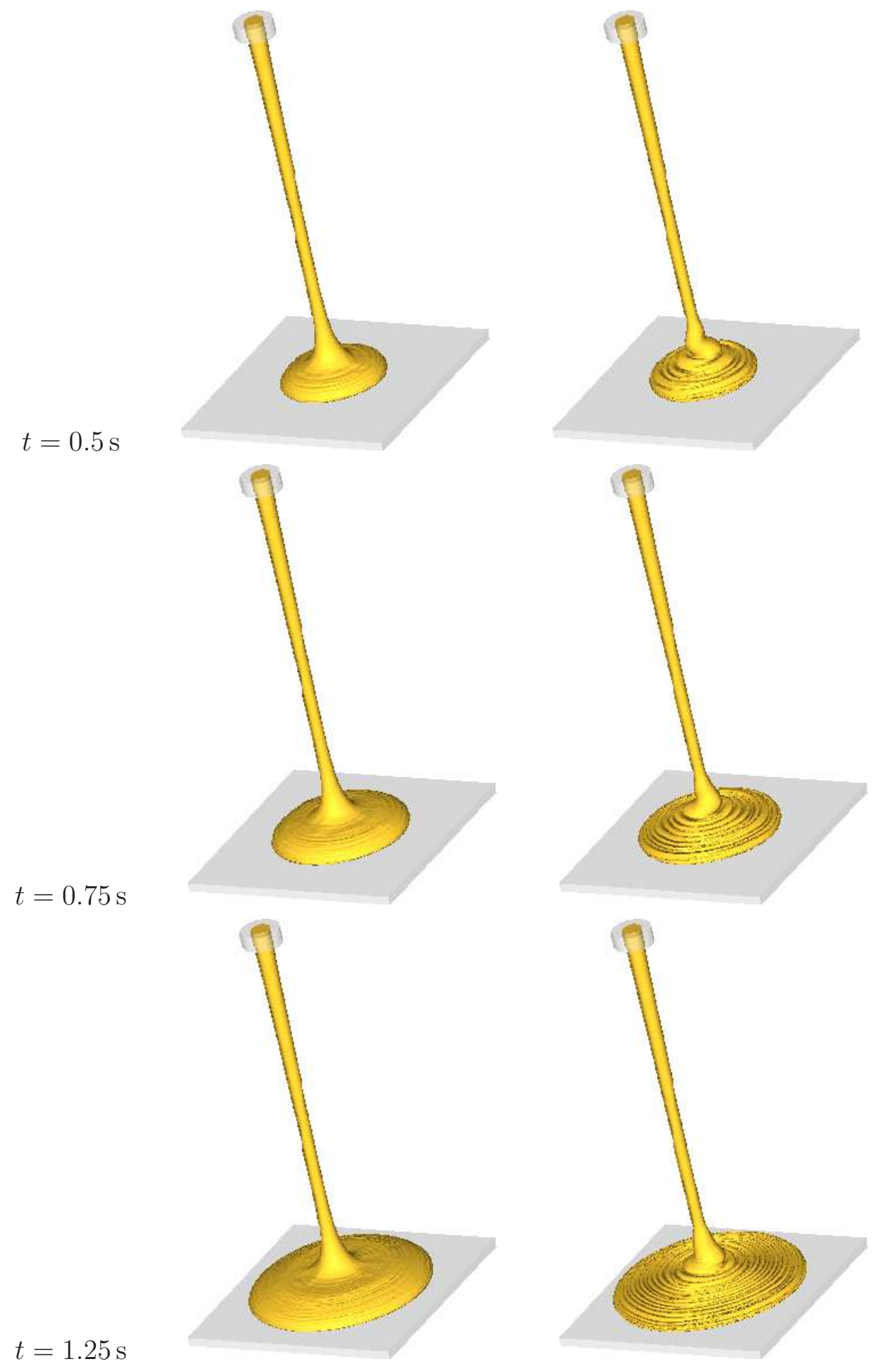

Figura 5.8: (cont.) Simulação para $R e=1.3$ (Problema 2). À esquerda, jato newtoniano; à direita, jato viscoelástico. 
newtonianc
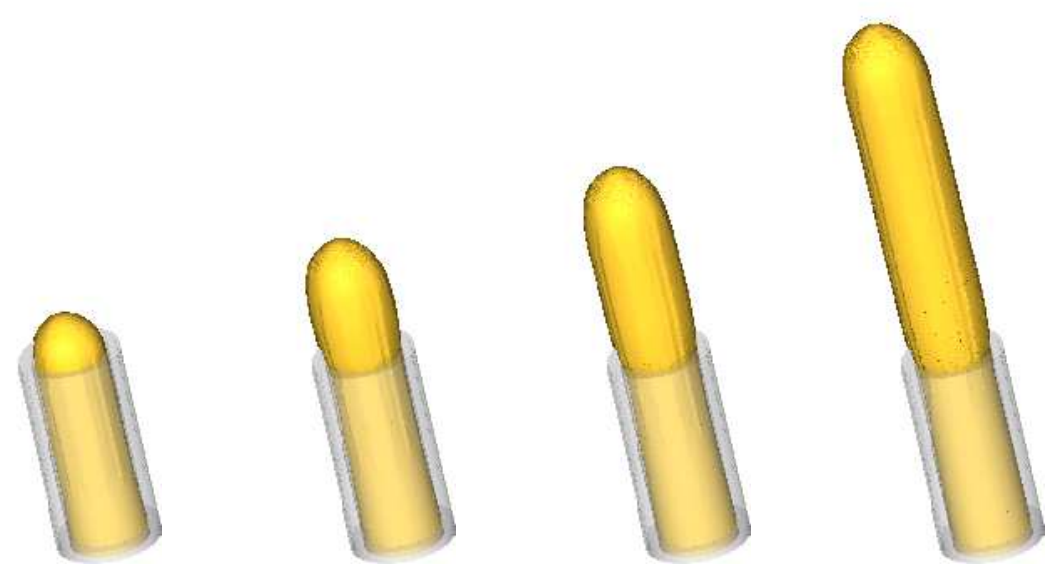

$W e=0.1$
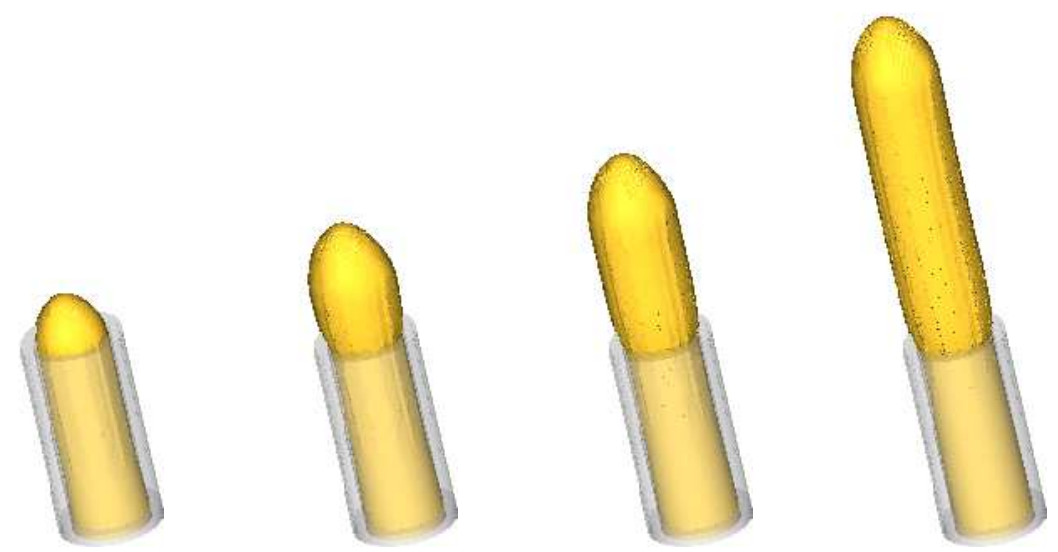

$W e=0.2$
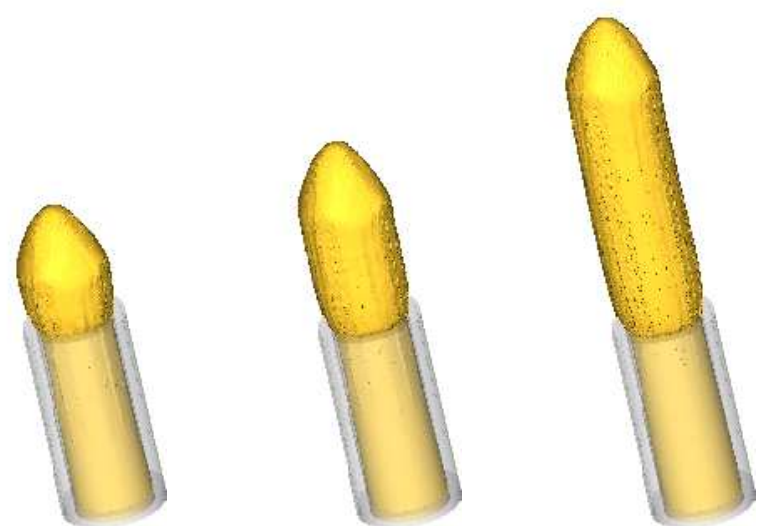

Figura 5.9: Visualização das simulações do inchamento do extrudado com aumento do número de Weissenberg, para instantes $t=5 \mathrm{~s}, 7.5 \mathrm{~s}, 10 \mathrm{~s}$ e $15 \mathrm{~s}$, respectivamente. 
$W e=0.3$
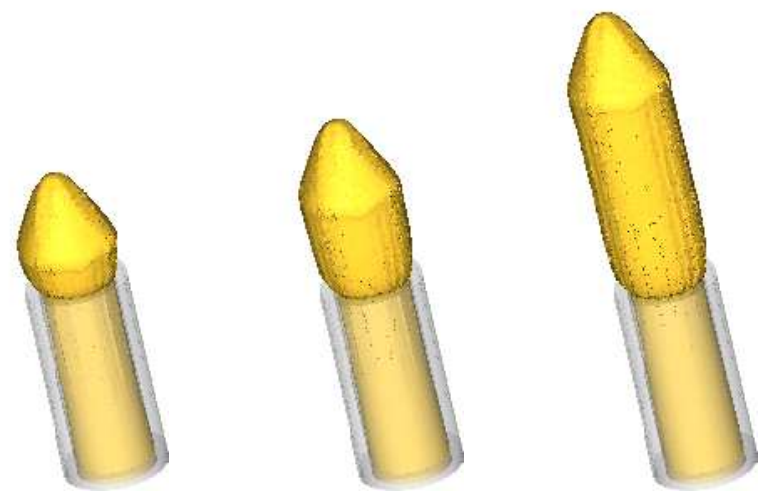

$W e=0.4$
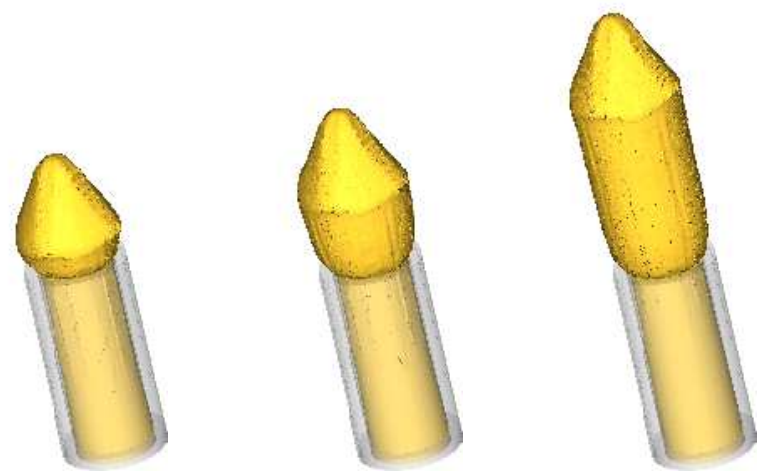

Figura 5.10: (cont.) Visualização das simulações do inchamento do extrudado com aumento do número de Weissenberg, para instantes $t=5 \mathrm{~s}, 7.5 \mathrm{~s}$, $10 \mathrm{~s}$ e $15 \mathrm{~s}$, respectivamente. 


\section{Capítulo 6}

\section{Considerações finais e conclusões}

Este trabalho apresentou os desenvolvimentos realizados para a obtenção de um método numérico para simular escoamentos incompressíveis com superfícies livres modelados pela equação constitutiva UCM. Foram apresentadas as equações governantes e a adimensionalização adotada, bem como a formulação EVSS. As condições de contorno para a velocidade e o cálculo do tensor não-newtoniano em contornos rígidos foram estudados em detalhes, juntamente com as condições de contorno na superfície livre para a pressão e a velocidade. A técnica explícita GENSMAC3D [6] foi discutida detalhadamente, bem como a discretização das equações utilizando o método de diferenças finitas. Apresentamos os resultados da validação do método numérico utilizando a solução analítica para escoamentos desenvolvidos em um tubo, sendo que a convergência foi demonstrada por meio do refinamento de malha computacional. Também foram apresentados resultados das simulações do fenômeno do jato oscilante e do fenômeno do inchamento do extrudado. Com relação ao jato oscilante, os resultados obtidos para jatos newtonianos concordaram com os resultados experimentais de Cruickshank e Munson [44]. Foi mostrado numericamente que a teoria de Cruickshank e Munson não se aplica para o fluido UCM. Os resultados obtidos nas simulações do inchamento do extrudado mostraram os efeitos da viscoelasticidade quando 
os jatos foram expelidos para a atmosfera. Entretanto, para os valores do número de Reynolds e do número de Weissenberg utilizados nas simulações do inchamento do extrudado não se verificou o fenômeno do inchamento retardado do extrudado. Existem diversos trabalhos na literatura a respeito desse problema, e podemos destacar os de Joseph e seus colaboradores [52-55], que mostram que tal fenômeno está associado à mudança do tipo de escoamento, que passa de supercrítico no interior do tubo para subcrítico após a extrusão.

Como proposta de trabalhos futuros, podemos mencionar o problema do inchamento retardado do extrudado acima citado, e outros conhecidos da literatura, como o splashing drop, filament stretching e squeeze flow. Recentemente o grupo de pesquisa em Matemática Computacional do ICMC obteve resultados para o primeiro problema, para escoamentos tridimensionais axissimétricos [32]. O problema do splashing drop ocorre quando uma gota de fluido é lançada de uma certa altura em um recipiente contendo uma certa quantidade de tal fluido. O problema do filament stretching caracteriza-se pelo estiramento de um bloco de fluido que encontra-se entre duas placas paralelas [57-61]. Inicialmente, as placas encontram-se em repouso e no instante $t=0$ passam a movimentar-se com uma velocidade exponencial. Para simular esse problema será necessário a implementação do movimento de fronteira rígida em função do deslocamento da placa no sistema Freeflow3D para resolver o problema tridimensional. Com estas alterações nos sistemas, acredita-se que seja possível o tratamento do squeeze flow que aparece em muitas aplicações industriais. Neste problema o fluido é colocado entre duas placas e estas movimentam-se de forma a comprimir o fluido entre elas [62$66]$. 


\section{Referências Bibliográficas}

[1] F. H. Harlow e J. E. Welch. Numerical calculation of time-dependent viscous imcompressible flow of fluid with free surface. Physics of Fluids, 8: 2182-2189, 1965.

[2] A. A. Amsden e F. H. Harlow. The SMAC method: a numerical technique for calculating incompressible fluid flow. Report LA 4370, Los Alamos Scientific Laboratory, 1970.

[3] M. F. Tomé. GENSMAC: A Multiple Free Surface Fluid Flow Solver. Ph.d. thesis, University of Strathclyde, Dept. of Mathematics, Glasgow, U.K., 1993.

[4] M. F. Tomé e S. McKee. GENSMAC: a computational marker-and-cell method for free surface flows. J. Comput. Phys., 110: 171-186, 1994.

[5] M. F. Tomé, B. R. Duffy, e S. McKee. A numerical technique for solving unsteady Non-Newtonian free surface flows. J. Non-Newtonian Fluid Mech., 62: 9-34, 1996.

[6] M. F. Tomé, A. Castelo, J. A. Cuminato, N. Mangiavacchi, e S. McKee. GENSMAC3D: A numerical method for solving unsteady threedimensional free surface flows. Intern. J. Numer. Meth. Fluids, 37: 747$796,2001$.

[7] A. Castelo, M. F. Tomé, C. N. L. César, S. McKee, e J. A. Cuminato. Freeflow: an integrated simulation system for three-dimensional free surface flows. Computing and Visualization in Science, 2: 199-210, 2000. 
[8] A. Beris, R. Armstrong, e R. Brown. Spectral finite-element calculations of the flow of a Maxwell fluid between excentric rotating cylinders. $J$. Non-Newtonian Fluid Mech., 22: 129-167, 1987.

[9] G. Mompean e M. Deville. Unsteady finite volume of Oldroyd-B fluid through a three-dimensional planar contraction. J. Non-Newtonian Fluid Mech., 72: 253-279, 1997.

[10] E. Brasseur, M. M. Fyrillas, G. C. Georgiou, e M. J. Crochet. The time-dependent extrudate-swell problem of an Oldroyd-B fluid with slip along the wall. J. Rheol., 42: 549-566, 1998.

[11] T. N. Philips e A. Williams. Comparison of creeping and inertial flow of an Oldroyd-B fluid though a planar and axisymmetric contraction. $J$. Non-Newtonian Fluid. Mech., 108: 25-47, 2002.

[12] F. T. Pinho, M. A. Alves, e P. J. Oliveira. Benchmark solutions for the flow of Oldroyd-B and PTT fluids in planar contractions. J. NonNewtonian Fluid Mech., 110: 45-75, 2003.

[13] N. Phan-Thien e R. I. Tanner. A new constitutive equation derived from network theory. J. Non-Newtonian Fluid. Mech., 2: 353-365, 1977.

[14] S. C. Xue, N. Phan-Thien, e R. I. Tanner. Three-dimensional numerical simulation of viscoelastic flows through planar contractions. J. NonNewtonian Fluid Mech., 74: 195-245, 1998.

[15] P. J. Oliveira e F. T. Pinho. Analytical solution for fully developed channel and pipe flow of Phan-Thien-Tanner fluids. J. Fluid. Mech., 387: 271-280, 1999.

[16] S. C. Xue, R. I. Tanner, e N. Phan-Thien. Three-dimensional numerical simulation of viscoelastic flows - predictability and accuracy. Comp. Meth. Appl. Mech. Engineering, 180: 305-331, 1999.

[17] M. A. Alves, F. T. Pinho, e P. J. Oliveira. Study of steady pipe and channel flows of a single-mode Phan-Thien-Tanner fluid. J. Non-Newtonian Fluid Mech., 101: 55-76, 2001. 
[18] X. L. Luo e R. I. Tanner. A streamline element scheme for solving viscoelastic flow problems part II: Integral constitutive models. J. NonNewtonian Fluid Mech., 22: 61-89, 1986.

[19] X. L. Luo e R. I. Tanner. Finite element simulation of long and short circular die extrusion experiments using integral models. Intern. J. Numer. Meth. Engineering, 25: 9-22, 1988.

[20] P. Olley, R. Spares, e P. D. Coates. A method for implementing timeintegral constitutive equations in commercial CFD packages. J. NonNewtonian Fluid. Mech., 86: 337-357, 1999.

[21] P. Olley. An adaptation of the separable K-BKZ equation for comparable response in planar and axisymmetric flow. J. Non-Newtonian Fluid. Mech., 95: 35-53, 2000.

[22] M. A. Hulsen, E. A. J. F. Peters, e B. H. A. A. Van Den Brule. A new approach to the deformation fields method for solving complex flows using integral constitutive equations. J. Non-Newtonian Fluid Mech., 98: 201-211, 2001.

[23] R. Keunings e M. J. Crochet. Numerical simulation of the flows of a viscoelastic fluid through an abrupt contraction. J. Non-Newtonian Fluid Mech., 14: 279-299, 1984.

[24] J. Yoo e Y. Na. A numerical study of the planar contraction flow of a viscoelastic fluid using the Simpler algorithm. J. Non-Newtonian Fluid Mech., 30: 89-106, 1991.

[25] T. N. Philips e A. Williams. Viscoelastic flow though a planar contraction using a semi-Lagrangian finite volume method. J. Non-Newtonian Fluid Mech., 87: 215-246, 1999.

[26] S. C. Xue, N. Phan-Thien, e R. I. Tanner. Numerical study of secondary flows of viscoelastic fluid in straight pipes by an implicit finite volume method. J. Non-Newtonian Fluid. Mech., 59: 191-213, 1995. 
[27] E. Mitsoulis. Three-dimensional non-newtonian computations of extrudate swell with the finite element method. Comput. Methods Appl. Mech. Engrg., 180: 333-344, 1999.

[28] V. Ngamaramvaranggul e M. F. Webster. Computation of free surface flows with a Taylor-Galerkin pressure correction algorithm. Intern. J. Numer. Meth. Fluids, 33: 961-992, 2000.

[29] V. Ngamaramvaranggul e M. F. Webster. Viscoelastic simulations of stick-slip and die-swell flows. Intern. J. Numer. Meth. Fluids, 36: 539$595,2001$.

[30] M. F. Tomé, N. Mangiavacchi, J. A. Cuminato, A. Castelo, e S. McKee. A numerical technique for solving unsteady viscoelastic free surface flows. J. Non-Newtonian Fluid Mech., 106: 61-106, 2002.

[31] M. F. Tomé, A. Castelo, , F. M. Federson, e J. A. Cuminato. A numerical method for solving the Oldroyd-B model for $3 \mathrm{~d}$ free surface flows. Mecánica Computacional, XXIII: 1931-1950, 2004.

[32] M. F. Tomé, L. Grossi, A. Castelo, J. A. Cuminato, S. McKee, e K. Walters. Die-swell, splashing drop and a numerical technique for solving the Oldroyd-B model for axisymmetric free surface flows. J. Non-Newtonian Fluid Mech., 141: 148-166, 2007.

[33] G. S. Paulo. Solução numérica do modelo PTT para escoamentos incompressivveis com superfícies livres. Tese de doutorado, ICMC-USP, São Carlos-SP, Brasil, 2006.

[34] G. S. Paulo, M. F. Tomé, e S. McKee. A marker-and-cell approach to viscoelastic free surface flows using the PTT model. In Press, 2007.

[35] D. Rajagopalan, R. C. Armstrong, e R. A. Brown. Finite element methods for calculation of steady, viscoelastic flow using constitutive equations with a Newtonian viscosity. J. Non-Newtonian Fluid Mech., 36: 159-192, 1990. 
[36] A. Chorin. Numerical solution of the navier-stokes equations. Mathematics of Computation, 2: 745-762, 1968.

[37] M. Crochet, A. R. Davis, e K. Walters. Numerical Simulation of NonNewtonian Flow. Elsevier, 1984.

[38] M. A. Alves, F. T. Pinho, e P. J. Oliveira. A convergent and universally bounded interpolation scheme for the treatment of advection. Intern. J. Numer. Meth. Fluids, 41: 47-75, 2001.

[39] G. K. Batchelor. An Introduction to Fluid Dynamics. Cambridge University Press, 1967.

[40] A. C. Brandi. Estratégias upwind e modelagem $\kappa-\epsilon$ para simulação numérica de escoamentos com superfícies livres em altos números de Reynolds. Dissertação de mestrado, ICMC-USP, São Carlos-SP, Brasil, 2005 .

[41] N. M. Ribe. A general theory of the dynamics of thin viscous sheets. $J$. Fluid Mech., 457: 255-283, 2002.

[42] N. M. Ribe. Periodic folding of viscous jets. Physical Review E, 68: Art. No. 036305 Part 2, 2003.

[43] N. M. Ribe. Coiling of viscous jets. In Proceedings of the Royal Society of London Series A - Mathematical Physical and Engineering Sciences, volume 460, pages 3223-3239, 2004.

[44] J. O. Cruickshank e B. R. Munson. Viscous fluid buckling of plane and axisymmetric jets. J. Fluid Mech., 113: 221-239, 1981.

[45] M. F. Tomé e Sean McKee. Numerical simulation of viscous flow: buckling of planar jets. Intern. J. Numer. Meth. Fluids, 29: 705-718, 1999.

[46] M. F. Tomé, L. Grossi, A. Castelo, J. A. Cuminato, N. Mangiavacchi, V. G. Ferreira, F. S. Sousa, e S. McKee. A numerical method for solving three-dimensional generalized Newtonian free surface flows. J. NonNewtonian Fluid Mech., 123: 85-103, 2004. 
[47] R. I. Tanner. A theory of die-swell. J. Polymer Science, 8: 2067-2078, 1970.

[48] M. E. Ryan e A. Dutta. A finite difference simulation of extrudate swell. In Proceedings of Second World Congress of Chemical Engineering, volume VI, pages 277-281, 1981.

[49] M. J. Crochet e R. Keunings. Finite element analysis of die-swell of a highly elastic fluid. J. Non-Newtonian Fluid Mech., 10: 339-356, 1982.

[50] R. Keunings. An algorithm for the simulation of transient viscoelastic flows with free surfaces. J. Comput. Phys., 62: 199-220, 1986.

[51] R. Keunings e D. Bousfield. Analysis of surface tension driven leveling in viscoelastic films. J. Non-Newtonian Fluid Mech., 22: 219-233, 1987.

[52] J. Y. Yoo e D. D. Joseph. Hyperbolicity and change of type in the flow of viscoelastic fluids through channels. J. Non-Newtonian Fluid Mech., 19: 15-41, 1985.

[53] D. D. Joseph, J. E. Matta, e K. Chen. Delayed die swell. J. NonNewtonian Fluid Mech., 24: 31-65, 1987.

[54] D. D. Joseph e C. Christodoulou. Independent confirmation that delayed die swell is a hyperbolic transition. J. Non-Newtonian Fluid Mech., 48: 225-235, 1993.

[55] M. Cloitre, T. Hall, C. Mata, e D. D. Joseph. Delayed-die swell and sedimentation of elongated particles in wormlike micellar solutions. $J$. Non-Newtonian Fluid Mech., 79: 157-171, 1998.

[56] R. I. Tanner. A theory of die-swell revisited. J. Non-Newtonian Fluid Mech., 129: 85-87, 2005.

[57] V. Tirtaatmadja e T. Sridhar. A filament stretching device for measurement of extensional viscosity. J. Rheol., 37: 1081-1102, 1993. 
[58] H. K. Rasmussen e O. Hassager. Three-dimensional simulations of viscoelastic instability in polymeric filaments. J. Non-Newtonian Fluid Mech., 82: 189-202, 1999.

[59] K. Foteinopoulou, V. G. Mavrantzas, e J. Tsamopoulos. Numerical simulation of bubble growth in newtonian and viscoelastic filaments undergoing stretching. J. Non-Newtonian Fluid Mech., 122: 177-200, 2004.

[60] M. F. Webster, H. Matallah, e K. S. Sujatha. Sub-cell approximations for viscoelastic flows-filament stretching. J. Non-Newtonian Fluid Mech., 126: 187-205, 2005.

[61] H. Matallah, M. J. Banaai, K. S. Sujatha, e M. F. Webster. Modelling filament stretching flows with strain-hardening models and sub-cell approximations. J. Non-Newtonian Fluid Mech., 134: 77-104, 2006.

[62] Y. Mochimaru. Fast squeezing flow of viscoelastic fluids. J. NonNewtonian Fluid Mech., 9: 157-178, 1981.

[63] N. Phan-Thien e H. T. Low. Squeeze-film flow of a viscoelastic fluid a lubrication model. J. Non-Newtonian Fluid Mech., 28: 129-148, 1988.

[64] B. Debbaut. Non-isothermal and viscoelastic effects in the squeeze flow between infinite plates. J. Non-Newtonian Fluid Mech., 98: 15-31, 2001.

[65] B. Debbaut e K. Thomas. Simulation and analysis of oscillatory squeeze flow. J. Non-Newtonian Fluid Mech., 124: 77-91, 2004.

[66] J. Engmann, C. Servais, e A. S. Burbidge. Squeeze flow theory and applications to rheometry: a review. J. Non-Newtonian Fluid Mech., 132: 1-27, 2005. 